Cite this: Phys. Chem. Chem. Phys., 2013, 15, 13737

Received 20th March 2013, Accepted 23rd April 2013 DOI: $10.1039 / \mathrm{c} 3 \mathrm{cp} 51213 d$

www.rsc.org/pccp

\title{
Redox and electrochemical water splitting catalytic properties of hydrated metal oxide modified electrodes $\dagger$
}

\author{
Richard L. Doyle, ${ }^{*}$ Ian J. Godwin, Michael P. Brandon and Michael E. G. Lyons
}

This paper presents a review of the redox and electrocatalytic properties of transition metal oxide electrodes, paying particular attention to the oxygen evolution reaction. Metal oxide materials may be prepared using a variety of methods, resulting in a diverse range of redox and electrocatalytic properties. Here we describe the most common synthetic routes and the important factors relevant to their preparation. The redox and electrocatalytic properties of the resulting oxide layers are ascribed to the presence of extended networks of hydrated surface bound oxymetal complexes termed surfaquo groups. This interpretation presents a possible unifying concept in water oxidation catalysis - bridging the fields of heterogeneous electrocatalysis and homogeneous molecular catalysis.

\section{In context}

Over the past 50 years considerable research efforts have been devoted to the realisation of efficient, economical and renewable energy sources. Metal oxide materials have played a large part in this drive with demonstrated applications at both the research and commercial level. Their use in areas such as batteries, fuel cells and water electrolysis has resulted in the development of materials with a diverse range of structural and chemical properties. In all cases, understanding the fundamental electrochemistry of the material can be invaluable for rational design and optimisation. This review focuses on the redox, charge transport and electrocatalytic properties of transition metal oxide electrodes as they pertain to the electrolytic splitting of water. Particular emphasis is placed on the nature of the active surface which is interpreted in terms of hydrated interlinked oxymetal complexes termed surfaquo groups. In this way, the review seeks to bridge the gap between heterogeneous electrocatalysis and homogeneous molecular catalysis for water oxidation, areas of considerable modern interest and activity.

\section{Introduction}

From coal to natural gas, the trend in fuel usage over time has tended towards an increase in the hydrogen content of the fuel. ${ }^{1}$

Trinity Electrochemical Energy Conversion \& Electrocatalysis (TEECE) Group, School of Chemistry and CRANN, University of Dublin Trinity College, Dublin 2, Ireland. E-mail: rdoyle5@tcd.ie, melyons@tcd.ie; Fax: +353 (0)1 671 2826; Tel: +353 (0)1 8962032

$\dagger$ This publication is based on a presentation given at the Smart Surfaces 2012: Solar \& Biosensor Applications conference in Dublin, Ireland.
This natural progression coupled with the dwindling supplies of traditional oil based fuels, increased awareness of $\mathrm{CO}_{2}$ emissions and soaring oil costs would lead one to believe that hydrogen gas will be a strong choice of fuel for the future. Indeed, hydrogen gas has been described as the ultimate clean energy source. ${ }^{2}$ Molecular hydrogen not only possesses a higher gravimetric energy density when compared with traditional fossil fuels but its combustion in energy devices such as fuel cells produces water as its only by product. ${ }^{3}$ Consequently, the use of hydrogen as a fuel has been proposed as the basis for a long term energy conversion and storage option, the so called hydrogen economy, consisting of the production of molecular hydrogen from nonfossil sources, its distribution and storage, and its cold combustion in a fuel cell to generate electricity. ${ }^{4}$

Of course when considering the environmental impact of an energy source, its method of production, and not just the products of its combustion, must also be taken into account. Currently, the dominant industrial method of hydrogen production is from the steam reforming of natural gas. ${ }^{5}$ This is an inherently environmentally offensive route due to both the consumption of natural gas as a fossil fuel, and to the production of $\mathrm{CO}_{2}$ as a product of the steam reforming process. In contrast, hydrogen generation via alkaline water electrolysis, using electricity generated from renewable sources, offers a clean, environmentally friendly and reliable route to the large scale hydrogen production required for a possible hydrogen economy. ${ }^{6-9}$ However, although the reaction of interest in an electrolysis cell is the generation of molecular hydrogen at the cathode, it is the generation of molecular oxygen at the anode which is the most energy intensive step in the overall 
electrolysis process. ${ }^{9-11}$ In practice, the efficiency of water electrolysis is limited by the large anodic overpotential of the oxygen evolution reaction (OER). Thus, understanding and optimising the oxygen evolution process is seen as one of the remaining grand challenges for both physical electrochemistry and energy science.

Over the past thirty years, considerable research effort has been devoted to the design, synthesis and characterization of OER anode materials with the aim of achieving useful rates of active oxygen evolution at the lowest possible overpotential, thereby optimizing the overall electrolysis process. Currently, the optimal OER anode materials are Dimensionally Stable Anode (DSA) type electrodes based on $\mathrm{RuO}_{2}$ and $\mathrm{IrO}_{2}$. These electrode materials, which were originally used in the production of chlorine, exhibit the lowest overpotentials for the OER at practical current densities. ${ }^{12}$ That said, despite their excellent electrocatalytic performance, the high cost of these materials, particularly iridium, and their poor long term stability in alkaline solution renders their widespread commercial utilisation both uneconomical and impractical. ${ }^{13}$ In light of these limitations, the oxides of the first row transition metals offer a compromise solution. Although they possess inferior electrocatalytic activity for the OER, their relatively low cost and long term corrosion resistance in alkaline solution makes them attractive OER anode materials. ${ }^{13-18}$ Consequently, a significant body of research exists on the application of non-noble transition metal oxides as OER anodes. Among the most promising materials are various intermetallic alloys, ${ }^{19-21}$ electrodeposited nickel, ${ }^{22-25}$ cobalt $^{26-28}$ and manganese oxides, ${ }^{29}$ spinels including nickelites, ${ }^{30-33}$ cobaltites $^{34-36}$ and ferrites, ${ }^{37,38}$ perovskites, ${ }^{39-42}$ and hematite photoanodes. ${ }^{43}$

An important practical and fundamental factor which should also be noted when considering OER anode materials is that, even in the case of the parent metal, the anodic OER always occurs at an oxidised surface. ${ }^{44}$ Hence, the electrocatalytic surface for such anodic processes is the external interface of the oxide film with the electrolyte solution. Accordingly, the physical and electrochemical properties of both noble and non-noble metal oxides have been studied in detail. Conway ${ }^{44}$ has provided a comprehensive account of the surface oxidation processes on $\mathrm{Pt}$ and $\mathrm{Au}$, examining the mechanism of anodic oxide formation from the sub-monolayer stage through to the growth of 3-dimensional bulk oxide films. Equally, the formation and electrochemistry of hydrated transition metal oxide films has been explored by Burke and Lyons ${ }^{45}$ in their early review on the subject. More recently, Lyons and coworkers ${ }^{46}$ have presented a thorough account of the redox and OER electrocatalytic properties of the hydrated metal oxide films in base. On the other hand, the electrochemical and catalytic behavior of compact anhydrous oxides, typically produced by the thermal decomposition of precursor metal salts, has been covered extensively by Trasatti. ${ }^{47,48}$

In the present review we focus our attention on three common types of OER anode materials: thermally prepared DSA type oxide electrodes, electrochemically prepared hydrous oxide electrodes, and electrodeposited bulk oxide/hydroxide electrodes. It is not our intention here to present an exhaustive review, as these synthetic methods together cover a vast range of the materials covered in the literature, instead we wish to emphasise some of the key concepts common to these materials and to the study of the OER in general. Firstly, we distinguish between the various synthetic routes and describe the main factors affecting the preparation of these materials; experimental parameters such as the annealing temperature and electrolyte concentration can have a dramatic effect on the nature and electrochemical properties of the oxide formed. Secondly, a brief outline of the redox properties of some representative first row transition metal and noble metal oxides is provided, paying particular attention to $\mathrm{Fe}, \mathrm{Ni}, \mathrm{Co}$, and, Ru and Ir. It is our belief that a study of the OER should not be separated from the surface electrochemistry of the OER anodes. In particular, we highlight how the redox characteristics and acid/base behaviour of the transition metal oxides can be ascribed to the presence of active octahedrally co-ordinated surface groups or surfaquo groups. Thirdly, we discuss how the application of fundamental electrochemical techniques such as steady-state polarization Tafel analysis, reaction order studies, open circuit potential decay curves (OCP) and electrochemical impedance spectroscopy (EIS) can form the basis of a comprehensive kinetic study of the OER, providing mechanistically significant kinetic parameters such Tafel slopes and reaction orders. In addition, we examine how the determination of turnover frequencies (TOF) can aid in the comparison of the electrocatalytic performance of different materials. Finally, various mechanistic aspects of the OER are summarised, paying particular attention to the concept of the surfaquo group. From this we postulate an OER mechanism incorporating our proposed surfaquo group representation which aims to bridge the ideas for the OER at metal oxides with water oxidation using molecular catalysts, and relate more general ideas in catalysis to electrochemical studies.

\section{The materials: transition metal oxide electrodes}

For the purposes of our discussion it is convenient to classify the transition metal oxide electrodes into three groups:

(i) DSA type electrodes.

(ii) Hydrous oxide electrodes.

(iii) Bulk oxide/hydroxide electrodes.

We may readily distinguish between these electrodes based on the nature of the oxide material, whether anhydrous or hydrous, amorphous or crystalline, compact or microdispersed, and their methods of preparation. In the following we give a brief overview of each type of electrode, with a focus on the most important factors affecting the preparation and electrocatalytic properties of the oxide layer.

\subsection{DSA type electrodes}

Activated metal anodes, more commonly known as Dimensionally Stable Anodes (DSA), consist of an underlying inert metal such as titanium, which is then coated with electrocatalytically 
active oxides of platinum group metals. Although the term DSA strictly refers to the underlying valve metal substrate, ${ }^{49}$ the complete assembly of the substrate and the catalytically active metal oxide coating is often referred to as a DSA electrode. ${ }^{50,51}$ DSA electrodes based on Ti-supported $\mathrm{RuO}_{2}$ and $\mathrm{Ru}$-based mixed oxide films, which compositionally resemble the industrial DSA used in commercial electrolyzers, have been studied extensively with early fundamental work being carried out by Beer, ${ }^{49}$ Trasatti, ${ }^{52,53}$ and De Nora ${ }^{54}$ to name but a few. Indeed such DSA type electrodes were central in showing that noble metal oxides show significant enhancements in electrolytic activity over that of the bare metal. ${ }^{49,51}$ Subsequently, various non-noble metal oxides and mixed oxides have also received considerable attention, among these materials the most notable include spinels such as $\mathrm{NiCo}_{2} \mathrm{O}_{4}{ }^{30-33}$ and $\mathrm{Co}_{3} \mathrm{O}_{4},{ }^{34-36}$ along with various perovskites. $^{39-42}$

The oxide materials used in DSA type electrodes are most commonly prepared by the thermal decomposition of precursor metal salts, usually chlorides or nitrates, and therefore typically consist of compact anhydrous oxides such as the rutile, perovskite and spinel materials discussed above, in which oxygen is present only as a bridging species between two metal cations and ideal crystals constitute tightly packed giant molecules. ${ }^{55-57}$ More recently, attention has also focused on sol-gel techniques, which are reported to give fewer residual impurities and thus, enhanced service lifetimes. ${ }^{50,58-61}$ That said, the popularity of the thermal method is understandable given the ease with which oxide and, in particular, mixed oxide electrodes may be prepared. In this procedure a metal salt or a predetermined mix of metal salts in the desired stoichiometry are dissolved in a suitable solvent, often water or isopropanol, and the resulting solution is concentrated until a 'paste' consistency has been achieved. The paste can then be painted onto the metal substrate and the solvent evaporated. Several coatings may be applied, taking care to evaporate the solvent after each application, until a sufficiently thick oxide loading has been obtained, typically 1-5 mg of oxide per $\mathrm{cm}^{2}{ }^{57}$ The electrode is then annealed at a given temperature for several hours to ensure complete decomposition of the metal salt to the metal oxide.

The choice of annealing temperature is probably the most important consideration when using a thermal preparation technique, having a demonstrated influence on the composition, morphology and electrocatalytic properties of the oxide film. It has been shown that the composition of the oxide film is closely dependent on the preparation temperature. ${ }^{62-64}$ On one hand, the temperature should be sufficiently high to allow the decomposition of the metal salt to the corresponding metal oxide, thereby minimising chloride/nitrate impurities. Trasatti and coworkers ${ }^{64}$ have shown that the level of chloride impurities in $\mathrm{RuO}_{2}$ films formed from $\mathrm{RuCl}_{3}$ decreases with increasing temperature in the range $300-800{ }^{\circ} \mathrm{C}$. However, depending on the annealing temperature different phases of the oxide layer may also form. For example, at temperatures $T>400{ }^{\circ} \mathrm{C} \mathrm{NiCo}_{2} \mathrm{O}_{4}$ decomposes to $\mathrm{NiO}$, resulting in enrichment of the $\mathrm{NiCo}_{2} \mathrm{O}_{4}$ surface with NiO. ${ }^{65,66}$ Similarly, $\mathrm{Co}_{3} \mathrm{O}_{4}$ decomposes to $\mathrm{CoO}$ at temperatures exceeding $900{ }^{\circ} \mathrm{C} .{ }^{65,67}$ In addition, the surface morphology of the oxide layer is also affected by the annealing temperature. Lodi et al. ${ }^{68}$ have observed that the surface roughness of compact $\mathrm{RuO}_{2}$ films increases with increasing preparation temperature.

In light of these temperature effects, it is not surprising that the electrocatalyitic properties of the oxide films are strongly influenced by the annealing temperature. In general, the electrocatalytic activity of thermally prepared oxides, such as $\mathrm{RuO}_{2}$ and $\mathrm{Co}_{3} \mathrm{O}_{4}$, tends to decrease with increasing annealing temperature. ${ }^{69}$ Trasatti and coworkers ${ }^{64}$ noted that the activity of pure $\mathrm{RuO}_{2}$ films, as expressed by the redox charge $Q^{*}$ which can be used as a measure of the surface concentration of active sites (Section 3.1), decreased as the temperature increased over the temperature range $300-800{ }^{\circ} \mathrm{C}$. On the other hand, the activity of a series of $\mathrm{Co}_{3} \mathrm{O}_{4}$ films decreased over the range $300-450{ }^{\circ} \mathrm{C}^{70,71}$ In the latter case, the reduction in electrocatalytic activity was associated with a decrease in excess oxygen in the film with increasing temperature. Furthermore, Iwakura et $a .^{72}$ have shown that lower Tafel slopes $\left(c a .40 \mathrm{mV} \mathrm{dec}{ }^{-1}\right)$ can be observed for $\mathrm{RuO}_{2}$ films fired at $450{ }^{\circ} \mathrm{C}$ than for similar oxides fired at $850{ }^{\circ} \mathrm{C}\left(\mathrm{ca} .70 \mathrm{mV} \mathrm{dec}{ }^{-1}\right)$. In the same way, the electrocatalytic activity of $\beta-\mathrm{MnO}_{2}$ was found to be optimised when thermally treated at $480{ }^{\circ} \mathrm{C} .{ }^{73}$ Using higher temperatures to anneal the oxide caused a decrease in activity, which was attributed to a decrease in the conductivity of the layer due to the formation of excess $\mathrm{Mn}_{2} \mathrm{O}_{3}$.

In addition, it is also important to highlight here the effect of surface morphology on the electrocatalytic activity of the thermally prepared oxide films. Trasatti and coworkers ${ }^{64,74}$ proposed that the electrocatalytic properties of $\mathrm{RuO}_{2}$-based electrodes are essentially related to the morphological structure of the surface rather than to its chemical composition. $\mathrm{RuO}_{2}$ films prepared using the brush coating technique outlined above typically exhibit a 'cracked' morphology, as shown in Fig. 1. ${ }^{75}$ However, Lodi et al. ${ }^{68}$ found that films with significantly more compact morphologies could be prepared using a dip coating procedure. Interestingly, the former authors observed distinct

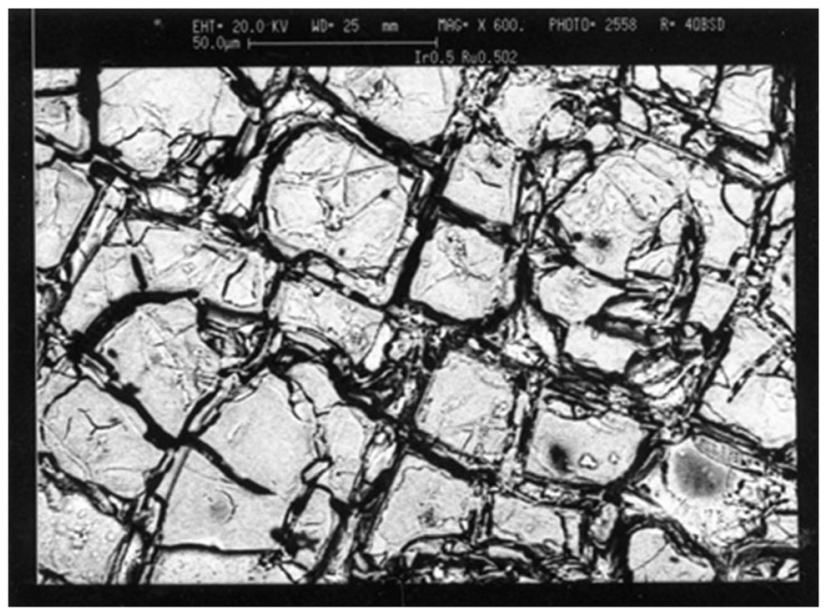

Fig. 1 SEM micrograph of a mixed $\mathrm{IrO}_{2} / \mathrm{RuO}_{2}$ coated Ti electrode showing the characteristic 'cracked' film morphology. 
differences in the Tafel slope depending on the level of 'compactness' of the surface oxide. ${ }^{64,74}$ The 'compact' or low defect films exhibited a Tafel slope of $c a .40 \mathrm{mV} \mathrm{dec}{ }^{-1}$ and this was observed to increase with increasing 'compactness'. On the other hand, Tafel slopes of $c a .30 \mathrm{mV} \mathrm{dec}^{-1}$ were observed for the more defective or 'cracked' films. The authors related these differences to changes in the value of $Q^{*}$, essentially observing an increase in Tafel slope with decreasing $Q^{*}$. That is, higher concentrations of active surface sites are associated with a more defective or 'cracked' morphology.

\subsection{Hydrous oxide electrodes}

In contrast to the compact anhydrous materials discussed above hydrous oxides are almost invariably prepared in aqueous environments resulting in more hydrated and dispersed materials, where oxygen is present not just as a bridging species between metal ions but also as $\mathrm{O}^{-}, \mathrm{OH}$ and $\mathrm{OH}_{2}$ species in coordinated terminal group form. Dispersion in the present context refers to the molecular level, i.e. microdispersion, and is usually due to the presence of strand, layer, tunnel or cage structures which allow not just small ions but also solvent molecules to permeate the oxide or hydroxide phase. In many cases, the latter materials, when in contact with aqueous media, contain considerable quantities of loosely bound and trapped water, plus, occasionally, electrolyte species. Indeed Burke and Lyons ${ }^{45}$ noted that with highly dispersed materials the boundary between the solid and aqueous phases may be somewhat nebulous as the two phases virtually intermingle. Furthermore, it is important to stress that the latter materials are very often deposited in the kinetically most accessible, rather than the thermodynamically most stable form. Hence, they are typically amorphous or only poorly crystalline and will indeed be prone to rearrangement in a manner that is directly influenced by factors such as temperature, $\mathrm{pH}$ and ionic strength.

Hydrous oxides may be prepared in a number of ways depending on the intended application of the material. Base precipitation is a routine procedure in gravimetric analysis. In this approach the transition metal ions are usually precipitated in hydrous form by the addition of base. The advantage of this method is its simplicity and oxide suspensions prepared in this manner may be used to examine double layer phenomena such as measurement of the potential of zero charge and ion adsorption phenomena. ${ }^{76}$ However, the investigation of the redox and electrocatalytic properties of these materials is less facile and requires that the hydrous oxide, ideally present in a uniform, homogeneous thin film form, be attached to some type of inert electronic conductor or supporting electrode. Various ways of preparing such coated chemically modified electrodes have been employed. One of the simplest approaches involves the direct anodization of the metal in an electrolyte using potentiostatic or galvanostatic techniques. This is frequently regarded as involving layer growth via a dissolution/precipitation hydrolysis type mechanism. This simple technique has been shown to be effective for some of the noble metals such as $\mathrm{Pt}^{77-79}$ and $\mathrm{Au},{ }^{80}$ but seems to be ineffective for $\operatorname{Ir}^{81-83}$ as the hydrous oxide dissolves under the highly anodic dc conditions that are successful in the case of Pt.

Probably the most versatile and convenient technique used to generate hydrous oxides in a form suitable for the real time determination of their redox switching and electrocatalytic behavior is potential cycling. ${ }^{84,85}$ In this method the potential of an electrode of the parent metal which may be noble or nonnoble is cycled repetitively between suitable lower and upper limits in an aqueous solution of appropriate $\mathrm{pH}$, often alkaline. The type of potential perturbation used for oxide growth, whether sinusoidal, square or triangular wave apparently makes little difference. Indeed the triangular wave is most convenient as changes in the current $v s$. potential response, the voltammogram, can be employed during the oxide growth reaction to monitor changes in redox behavior associated with the latter. ${ }^{86}$ That said, Arvia and co-workers ${ }^{87}$ contend that a repetitive square wave potential pulse is very effective for the controlled generation of relatively thick hydrated metal oxy-hydroxide films.

In Fig. 2a, a series of cyclic voltammograms showing the growth of the hydrous layer on an Fe electrode are presented as an example of the usefulness of this method. Using the triangular wave method or cyclic voltammetry, the growth of
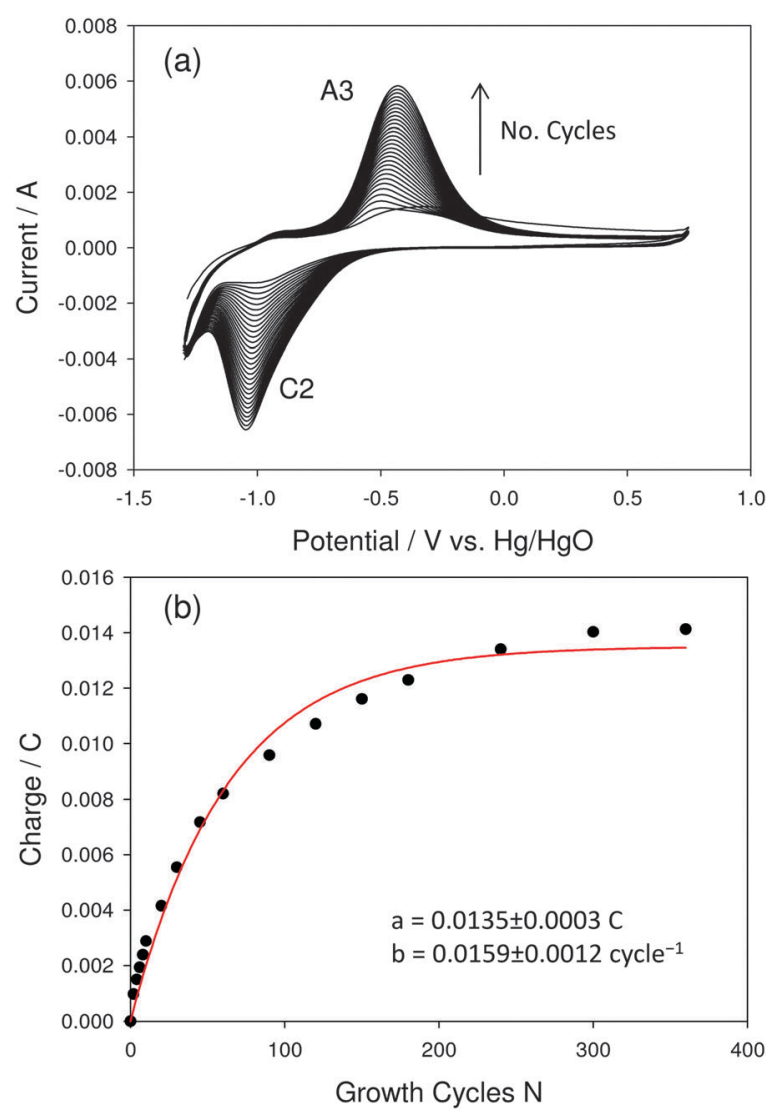

Fig. 2 (a) Cyclic voltammograms recorded during the growth of a hydrous iron oxide film in $0.5 \mathrm{M} \mathrm{NaOH}$ between the potential limits of $-1.30 \mathrm{~V}$ and $0.75 \mathrm{~V}$ at $350 \mathrm{mV} \mathrm{s}^{-1}$. (b) The voltammetric charge capacity $Q$ under the $A 3$ peak as a function of the number of growth cycles $N$ for a hydrous iron oxide film prepared $0.5 \mathrm{M} \mathrm{NaOH}$. The growth curve simulated using eqn (1) is presented as a continuous line. 
the hydrous oxide film on the metallic support can be readily monitored by following the development of the set of A3/C2 redox peaks, corresponding to the main charge storage process on the surface, as a function of either time or the number of cycles $N$. It should be noted that the integrated voltammetric charge $Q$, the area under each voltammetric wave, is directly related to the oxide layer thickness $L$, and we have shown previously that the variation in the value of $Q$ as a function of $N$ can be fitted quantitatively using the following expression,

$$
Q=a[1-\exp (-b N)]
$$

Experimentally it is found that the charge tends toward a constant limiting value as the number of cycles increases, as shown in Fig. $2 \mathrm{~b}$. The decrease in oxide growth rate $\mathrm{d} Q / \mathrm{d} N$ with increasing film thickness has been attributed to the increasing inhibition of water and hydroxide ion transfer to the inner region of the oxide layer with increasing hydrous oxide thickness. ${ }^{46}$

It has been noted that potential cycling causes roughening of the surface of noble metals such as platinum under certain conditions. $^{88,89}$ Certainly, the oxide layers prepared in this manner on the surface of Fe electrodes exhibit significant surface roughness, as can be observed clearly from the SEM micrograph presented in Fig. 3a. The oxide/solution interface of such oxide layers is thought to consist of an inner compact anhydrous layer $\mathrm{MO}_{x}$ and an outer microdispersed hydrous layer of general form $\mathrm{MO}_{a}(\mathrm{OH})_{b}\left(\mathrm{OH}_{2}\right)_{c}$. This is the duplex layer model proposed by Burke and coworkers ${ }^{90,91}$ and depicted graphically in Fig. 3b. In this model, the ions of the inner region are held in place by a rigid network of polar covalent bonds through which ionic transport is difficult whereas charge percolation proceeds comparatively easily and quickly through the outer, hydrous, polymeric oxide region. The kinetics of the latter process can be quantitatively determined using simple techniques such as cyclic voltammetry (Section 4). On repetitive cycling, the porous outer layer increases in thickness at the expense of the underlying metal, the mechanism of which has been described by Burke and Lyons, ${ }^{84}$ and Pickup and Birss, ${ }^{93}$ and recently reviewed by Lyons and coworkers. ${ }^{46}$

The extent of hydrous oxide growth is known to depend strongly on the values chosen for the upper and lower limit of the potential sweep as well as the cycling frequency and the solution $\mathrm{pH}$. The marked dependence of oxide growth rate on the lower limit of the potential sweep is indicative of the essential role that partial reduction of the anhydrous oxide layer plays in the production of a thick deposit. Thus, with platinum $^{94}$ and gold, ${ }^{95}$ two metals where oxide monolayer behaviour is well defined, the optimum lower limit lies at a potential value at, or below, the value of the monolayer oxide reduction peak. In a similar manner, Lyons and coworkers ${ }^{96}$ have recently examined the effect of the lower potential limit on the growth of hydrous Fe oxide films. As shown in Fig. 4a, the extent of hydrous oxide growth on an Fe electrode, as measured by the charge capacity $Q$, is maximised when the potential sweep is reversed at the $\mathrm{C} 1$ peak potential, corresponding to the reduction of the compact anhydrous layer.

Partial reduction of the compact oxide layer apparently facilitates rearrangement of oxycation species at the metal surface, leaving it in a somewhat disrupted state. On subsequent re-oxidation of the partially reduced metal surface the compact layer is restored but the outer region of the compact film is present in a more dispersed form and on further reduction the latter material becomes incorporated into the hydrated outer layer. ${ }^{46}$

The upper limit of the potential sweep also has an important effect on the rate of oxide growth. Lyons and coworkers ${ }^{84,96}$ have found that the efficiency of hydrous oxide growth increases for more anodic upper potential limits. This is shown clearly in Fig. 4b where the charge capacity $Q$ for hydrous Fe oxide films is observed to increase as a function of the upper potential limit. The importance of this parameter probably lies in the fact that it extends oxygen penetration into the outer regions of the metal lattice resulting in thickening of the compact layer. Indeed, it has been noted that the upper limit must be sufficiently anodic that compact oxide formation exceeds significantly the single monolayer level so that on subsequent reduction, a disturbed, highly disordered layer of metal atoms is prepared on the electrode surface thereby facilitating hydrous oxide growth. ${ }^{46}$ In addition, employing more anodic upper potentials may help to generate a slight expansion and stress associated disruption at the metal-oxide interface and also enable the uptake of a slight excess of oxygen by the oxide phase. Another effect of this parameter which
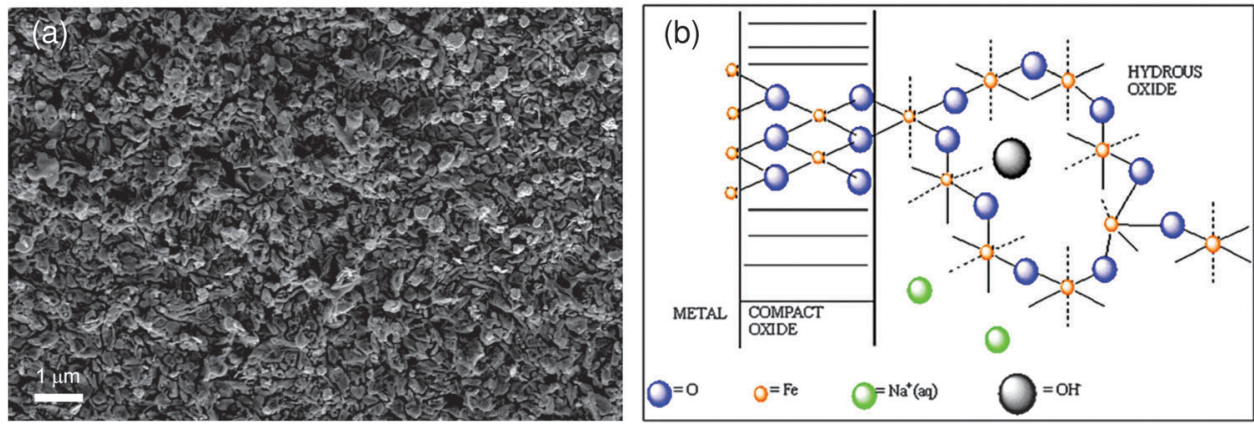

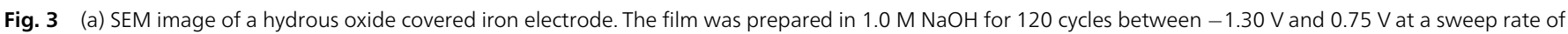
$400 \mathrm{mV} \mathrm{s}^{-1}$. (b) Schematic representation of the Burke-O'Sullivan Duplex Layer Model of the oxide/solution interface. 

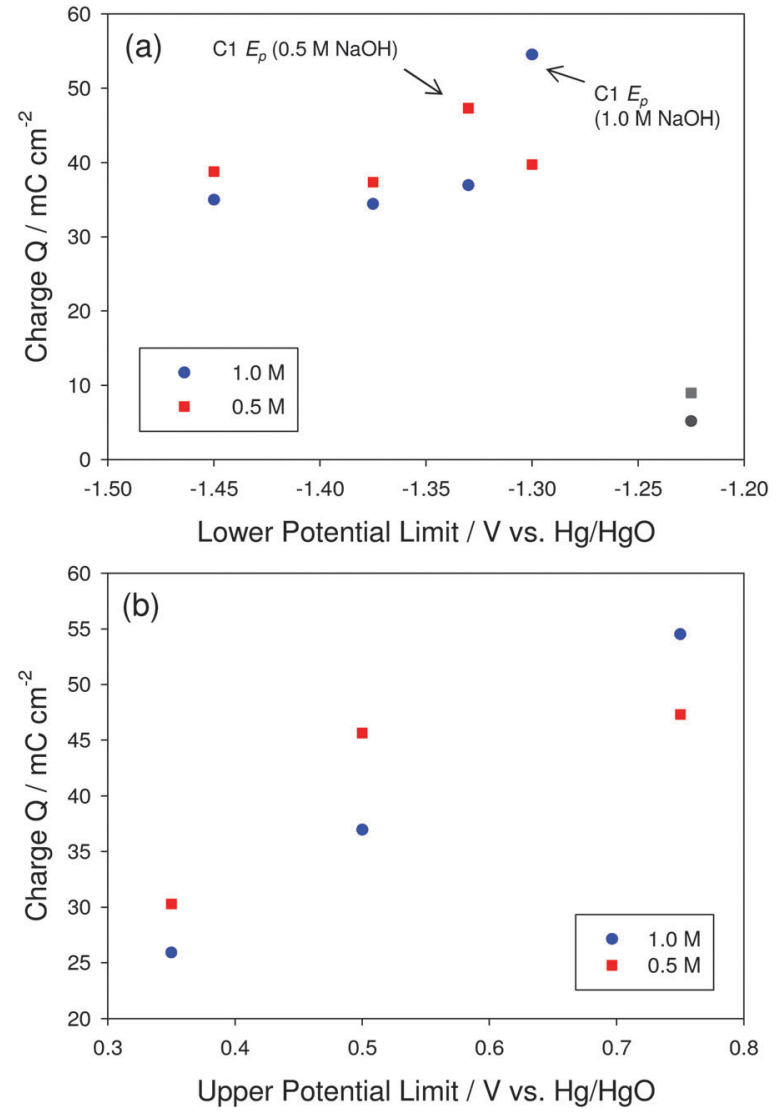

Fig. 4 The voltammetric charge capacity $Q$ under the $A 3$ peak plotted as a function of (a) the lower potential limit and (b) the upper potential limit for hydrous iron oxide films prepared using 120 growth cycles in 0.5 and $1.0 \mathrm{M}$ $\mathrm{NaOH}$ with a sweep rate of $350 \mathrm{mV} \mathrm{s}^{-1}$. Note: the $\mathrm{C} 1$ peak potentials, corresponding to the reduction of the compact inner oxide layer, were $-1.33 \mathrm{~V}$ and $-1.30 \mathrm{~V}$ in 0.5 and $1.0 \mathrm{M} \mathrm{NaOH}$ respectively.

should be considered is the fact that the more anodic the value of the upper potential limit the more difficult the reduction of the compact anhydrous oxide layer becomes. ${ }^{96}$ This behavior is likely due to increased passivation of the electrode surface at the more anodic potentials. Indeed, it has been established that the oxide reduction reaction involves a nucleation process which occurs less rapidly as the film formation potential is raised. ${ }^{97}$ Consequently, Lyons and coworkers ${ }^{96}$ have observed that a relationship exists between the upper and lower limit such that the optimal lower limit necessary to achieve maximum hydrous oxide growth is determined by the value chosen for the upper limit of the potential sweep.

On the other hand, it is not just the magnitude of the potential applied to the electrode which should be considered, the time spent in the critical regions of the upper and lower limit of the sweep also appears to be important. This is often demonstrated by a sharp maximum in the charge capacity $Q v s$. growth sweep rate curve for many transition metals such as Fe, ${ }^{17,84}$ as shown in Fig. 5a. The decrease in the efficiency of multilayer oxide growth with increasing sweep rate for values greater than the optimum, $340 \mathrm{mV} \mathrm{s}^{-1}$ in the case of Fe in $1.0 \mathrm{M}$ $\mathrm{NaOH}$, may possibly be due to a hysteresis effect. Lyons ${ }^{84}$ has
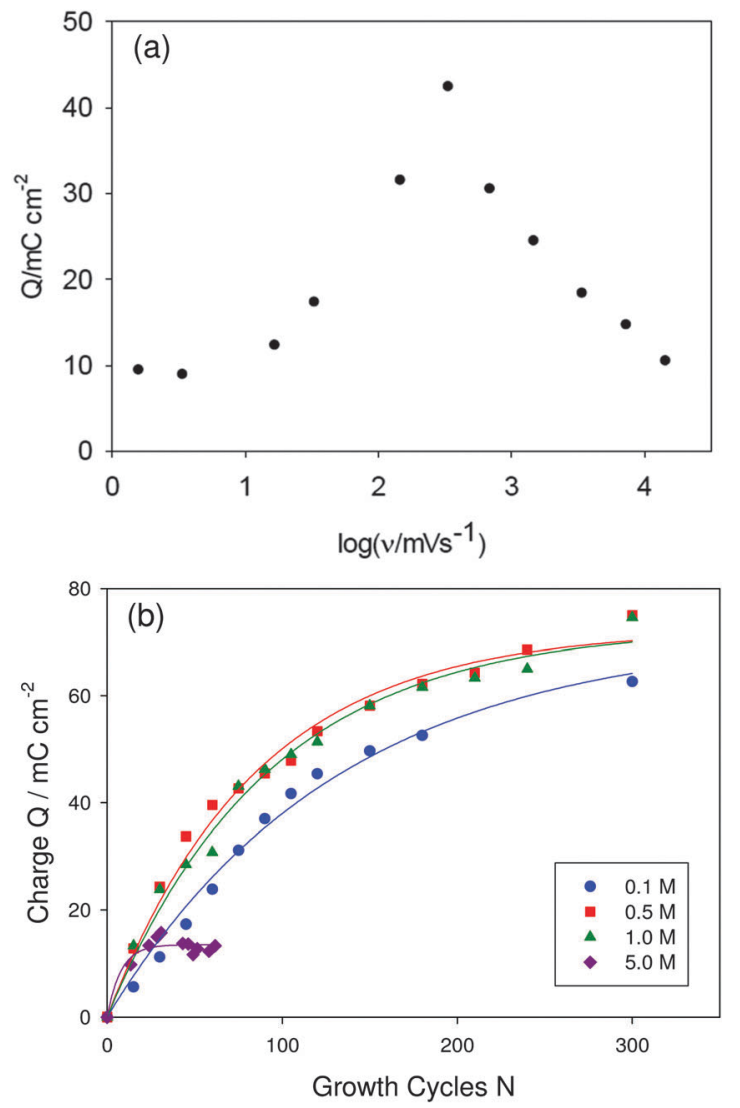

Fig. 5 (a) The effect of oxide growth sweep rate on the voltammetric charge capacity $Q$ determined for a hydrous iron oxide film prepared using 30 growth cycles in $1.0 \mathrm{M} \mathrm{NaOH}$. (b) $Q$ plotted as a function of the number of growth cycles $N$ for hydrous iron oxide films prepared in $0.1,0.5,1.0$ and $5.0 \mathrm{M} \mathrm{NaOH}$. The growth curves simulated using eqn (1) are shown as continuous lines.

shown that the voltammetric peak potentials for the outer hydrous and compact oxide shift with increasing sweep rate. The oxidation peak potential shifts to more anodic values and the reduction peaks shift to more cathodic values. Hence a certain amount of hysteresis exists between formation and reduction of the compact and hydrous films. It is quite probable that the reduction region of the film shifts gradually to more cathodic potentials as the sweep rate increases, which results in insufficient reduction or rupture of the anhydrous layer. Conversely, the decrease in oxide growth rate below $340 \mathrm{mV} \mathrm{s}^{-1}$ may be due to excessive reduction of the compact layer. Thus, the important role of sweep rate in the development of the charge storage capacity of the surface film clearly demonstrates the importance of kinetic factors in the potential cycling route to hydrous oxide film formation.

Furthermore, Lyons and coworkers ${ }^{17,84,96}$ have shown that the rate of hydrous oxide growth is dependent on the electrolyte concentration. The latter authors examined the growth of hydrous Fe oxides in a range of base concentrations and the resultant growth profiles are presented in Fig. 5b. During the initial stages of layer growth (small $N$ ), the growth rate is more rapid in the more concentrated $\left(5.0 \mathrm{~mol} \mathrm{dm}^{-3}\right)$ as opposed to the more dilute $\left(0.1 \mathrm{~mol} \mathrm{dm}^{-3}\right)$ hydroxide solutions. 
However, the growth rate decreases quite rapidly with increasing $N$ in the more concentrated medium, whereas the growth rate is less affected with increasing $N$ in the more dilute solutions. Evidently, increased hydroxide ion activity suppresses hydroxide dissociation and/or favours adsorption of this species. Lyons and coworkers ${ }^{46}$ note that this will result in the inhibition of crystallization of the hydrous oxide layer, and the resulting more amorphous film will be more effective in excluding water from the inner region of the oxide film, thereby inhibiting the growth of the microdispersed hydrous layer. Certainly, as can be seen from Fig. 5b, significantly greater redox capacities may be obtained for hydrous oxide layers prepared in the lower base concentrations whereas only limited development of the hydrous layer can be achieved in very concentrated base, with inhibition of hydrous oxide growth becoming apparent after only 20-30 growth cycles in $5.0 \mathrm{~mol} \mathrm{dm}{ }^{-3} \mathrm{NaOH}$.

In addition to the latter effect, the base concentration used to prepare the hydrous oxide can also influence the electrocatalytic properties of the film. Lyons and coworkers ${ }^{96,98,99}$ found that hydrous Fe oxide layers exhibited distinct Tafel slopes for the OER depending on the concentration of $\mathrm{NaOH}$ used to prepare the film. In particular, it was observed that hydrous layers grown in high base concentrations had an associated Tafel slope of $c a .60 \mathrm{mV} \mathrm{dec}{ }^{-1}$ whereas those grown in low base concentrations $(<1.0 \mathrm{M})$ exhibited a Tafel slope of ca. $40 \mathrm{mV} \mathrm{dec}{ }^{-1}$. This differing Tafel behaviour was attributed to the fact that more dehydrated layers, with differing electrocatalytic properties, would be formed in the more concentrated base solutions. Indeed, the latter authors noted that by purposely dehydrating the hydrous Fe oxide layer, a film with a characteristic

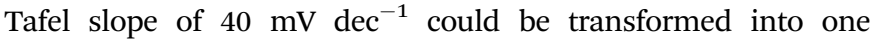
displaying a $60 \mathrm{mV} \mathrm{dec}{ }^{-1}$ slope. $^{96,98}$

\subsection{Bulk oxide/hydroxide electrodes}

Bulk oxide/hydroxide electrodes have been studied extensively in the literature. This work has, in a large part, been prompted by the uses these materials have found in energy storage technology. For instance, transition metal oxides based on nickel, ${ }^{100-108}{\text { cobalt },{ }^{109-111} \text { manganese }}^{112-121}$ and iron ${ }^{122-126}$ have been identified as possible electrode materials for electrochemical supercapacitors, whereas $\mathrm{Ni}(\mathrm{OH})_{2}$ is widely used as a cathode material in $\mathrm{Ni}-\mathrm{Cd}^{127-130}$ and $\mathrm{Ni}-$ Metal hydride ${ }^{131,132}$ batteries. In addition, it has long been recognised that nickel, cobalt and their various mixed oxides exhibit promising electrocatalytic properties for the electrochemical oxidation of water. ${ }^{133-142}$ Indeed, in recent years, the cobalt catalyst film (CoCF) developed by Nocera and co-workers ${ }^{26}$ has attracted much attention because of its efficiency at neutral $\mathrm{pH}$, self assembly from low cost materials and for its self repair mechanism. ${ }^{143}$ Moreover, as noted by Dau et al. ${ }^{10}$ the success of this material may have initiated a revival of bulk metal oxide/hydroxide materials as catalysts for electrochemical water oxidation.

In the present context, the designation bulk oxide/hydroxide electrode refers to a metal oxide or hydroxide film deposited on a conducting substrate. While these oxide materials may be more compact than the microdispersed hydrous oxides discussed previously, they often lack the long-range bonding order of the more crystalline materials employed in DSA type electrodes. ${ }^{10}$ Several physical and chemical methods of preparing these films have been described in the literature. These include sol-gel processes, ${ }^{114,144-146}$ hydrothermal synthesis, ${ }^{147}$ chemical precipitation, ${ }^{117,148,149}$ vacuum evaporation, ${ }^{150}$ mechanical grinding, ${ }^{151}$ pyrogenation, ${ }^{152}$ electrostatic spray deposition $^{153}$ and electron beam deposition, ${ }^{154}$ as well as various sputtering ${ }^{155,156}$ and vapour deposition techniques. ${ }^{157,158}$ However, possibly the simplest and most versatile of the existing synthetic approaches is electrochemical deposition. In this method, the conducting substrate is coated either anodically or cathodically with a metal oxide or hydroxide film from an electrolyte solution containing an appropriate metal salt. Various experimental techniques including potentiostatic, ${ }^{107,108,159}$ galvanostatic, ${ }^{121,126,160-164}$ potentiodynamic, ${ }^{29,165-167}$ and pulse techniques $^{121,168,169}$ have been used and depending on the specific parameters, metal oxides and hydroxides of different nanostructures, such as nanoparticles, ${ }^{170}$ nanoneedles ${ }^{159}$ and nanorods, ${ }^{29,164}$ morphology and composition may be prepared. In this way, electrochemical techniques offer unique control over the physical and chemical properties of the oxide/hydroxide layers being deposited.

Specifically, it is known that the electrochemical method employed for the electrodeposition can have a strong influence on the composition of the oxide/hydroxide film. For example, $\mathrm{Ni}(\mathrm{OH})_{2}$ is most commonly prepared galvanostatically or potentiostatically from an aqueous solution containing nickel nitrate. ${ }^{159-164}$ Under such circumstances the deposition chemistry involves the direct or indirect reduction of the nitrate ion to either nitrous acid, hydroxylamine or the nitrite ion. ${ }^{160,171,172}$ In all cases the hydroxyl ion is generated, resulting in the formation of either $\alpha-\mathrm{Ni}(\mathrm{OH})_{2}$ or $\beta-\mathrm{Ni}(\mathrm{OH})_{2}$ according to eqn (2),

$$
\mathrm{Ni}_{\mathrm{aq}}{ }^{2+}+2 \mathrm{OH}^{-} \rightarrow \mathrm{Ni}(\mathrm{OH})_{2}
$$

Alternatively, $\mathrm{Ni}(\mathrm{OH})_{2}$ may also be formed according to eqn (2) using cyclic voltammetry in an aqueous acetate buffer solution containing $\mathrm{Ni}^{2+}$ and $\mathrm{OH}^{-}$ions. However, under these conditions it has been noted that the following process may occur as the potential is cycled anodically, ${ }^{167,173}$

$$
\begin{gathered}
\mathrm{OH}^{-} \rightarrow \mathrm{OH}_{\mathrm{ads}}+\mathrm{e}^{-} \\
\mathrm{Ni}_{\mathrm{aq}}{ }^{2+}+\mathrm{H}_{2} \mathrm{O}+\mathrm{OH}_{\mathrm{ads}} \rightarrow \mathrm{NiOOH}+2 \mathrm{H}^{+}
\end{gathered}
$$

Additionally, the following solution processes may also occur, ${ }^{167,173}$

$$
\begin{gathered}
\mathrm{Ac}^{-}+\mathrm{H}_{2} \mathrm{O} \rightarrow \mathrm{HAc}+\mathrm{OH}^{-} \\
\mathrm{Ni}_{\mathrm{aq}}{ }^{2+}+\mathrm{OH}^{-} \rightarrow \mathrm{NiOH}_{\mathrm{aq}}{ }^{+} \\
\mathrm{NiOH}_{\mathrm{aq}}^{+}+\mathrm{OH}_{\mathrm{ads}} \rightarrow \mathrm{NiOOH}+2 \mathrm{H}^{+}
\end{gathered}
$$

where $\mathrm{Ac}=\mathrm{CH}_{3} \mathrm{COO}^{-}$. Hence, both $\mathrm{Ni}(\mathrm{OH})_{2}$ and NiOOH may well be formed during the course of the potential sweep perturbation.

A similar dependence of the composition of the oxide/ hydroxide film on the experimental method has also been observed for $\mathrm{Mn}$ and Co based materials. Xiao et al. ${ }^{121}$ anodically deposited 
manganese oxide films using galvanostatic and pulsed current methods. X-ray diffraction (XRD) analysis indicated that the galvanostatic method produced $\mathrm{Mn}_{3} \mathrm{O}_{4}$ films, whereas the films produced using the pulsed current technique contained a mix of $\mathrm{Mn}_{3} \mathrm{O}_{4}$ and $\mathrm{MnOOH}$. On the other hand, Castro et al. ${ }^{28,142}$ compared the anodic deposition of cobalt oxide on nickel substrates using potentiostatic and potentiodynamic techniques. Using cyclic voltammetry and X-ray photoelectron spectroscopy (XPS) the authors observed that under potentiodynamic conditions the cobalt oxide being deposited mixed with the surface nickel oxide, which was formed during the voltammetric scan, to produce a $\mathrm{NiCo}_{2} \mathrm{O}_{4}$ film. Conversely, pure $\mathrm{Co}_{3} \mathrm{O}_{4}$ was deposited under potentiostatic conditions.

Furthermore, variations in the different experimental parameters, such as electrolyte composition, applied potential, electrode substrate and sweep rate for potentiodynamic deposition, have also been shown to affect the properties of the electrodeposited oxide/hydroxide films. Nagarajan et al. ${ }^{126}$ found that the addition of chitosan to the plating solution increases the adhesion of $\gamma-\mathrm{Fe}_{2} \mathrm{O}_{3}$ films to the substrate. Similarly, the use of a water-ethanol mixture has been shown to enhance the adhesion of $\mathrm{Ni}(\mathrm{OH})_{2}$ films to the metal support. ${ }^{171,172}$ On the other hand, Yousefi et $a .^{164}$ examined the effect of dissolved oxygen content on the cathodic galvanostatic deposition of manganese oxide from $\mathrm{Mn}\left(\mathrm{NO}_{3}\right)_{2}$ solutions. They determined from the use of XRD and XPS that $\mathrm{Mn}_{3} \mathrm{O}_{4}$ was preferentially formed in an oxygen rich environment whereas, $\mathrm{Mn}(\mathrm{OH})_{2}$ films were produced when the plating solution was degassed with $\mathrm{N}_{2}$.

The influence of the deposition potential on the thickness of anodically formed $\mathrm{MnO}_{2}$ was reported by Cherchour et al. ${ }^{159}$ The thickness of the deposited film increased with applied potential, reaching a maximum at the $\mathrm{MnO}_{2}$ anodic peak potential. Further increases in potential beyond the $\mathrm{Mn}$ (II)/ Mn(Iv) oxidation peak had no effect on the thickness of the as formed film, although thinner films were formed when the deposition was carried out at potentials where oxygen was evolved. Alternatively, Nocera and co-workers ${ }^{26}$ have noted that CoCF deposited at potentials below the onset of oxygen evolution are microscopically smooth, whereas films deposited at potentials which promote water oxidation have a nodular surface. Moreover, extended X-ray absorption fine structure (XAFS) analysis indicated that the former films exhibit greater long-range bond order than those formed at the higher potentials associated with oxygen evolution.

In addition, the effect of sweep rate on the potentiostatic deposition of $\mathrm{Ni}(\mathrm{OH})_{2}$ films was investigated by Lyons and coworkers. ${ }^{167}$ Cyclic voltammograms recorded for films prepared at different sweep rates, with all other experimental conditions the same, are presented in Fig. 6. Evidently, the timescale adopted for the layer deposition can affect the composition of the deposited oxide film. Both the $\alpha / \gamma-\mathrm{Ni}(\mathrm{II} / \mathrm{III})$ and the $\beta / \beta-\mathrm{Ni}(\mathrm{II} / \mathrm{III})$ redox transitions, as described by the Bode cycle $^{173}$ and outlined below in Section 3.3, were observed for the layers deposited at slow sweep rates/long timescales. This is clear from the two anodic peaks and the cathodic doublet

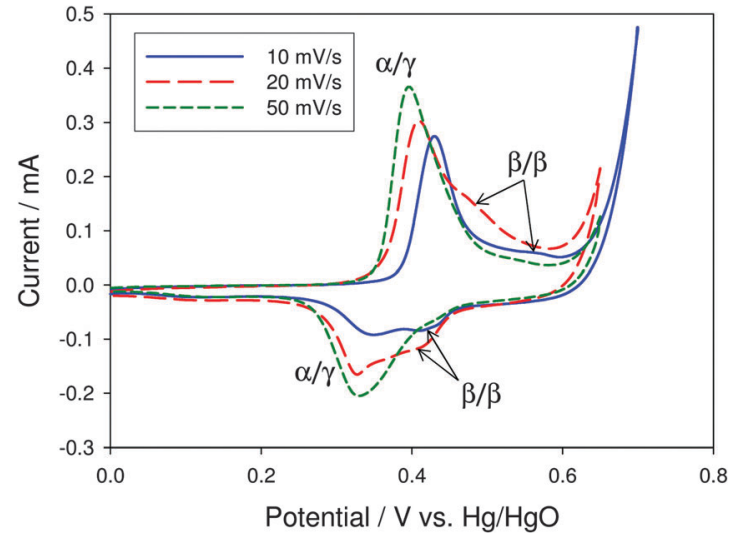

Fig. 6 Cyclic voltammograms recorded in $1.0 \mathrm{M} \mathrm{NaOH}$ at $40 \mathrm{mV} \mathrm{s}^{-1}$ for a $\mathrm{Au}$ electrode modified with nickel hydroxide films deposited using 30 growth cycles performed at a sweep rate of 10,20 and $50 \mathrm{mV} \mathrm{s}^{-1}$.

observed for the layer prepared at $10 \mathrm{mV} \mathrm{s}^{-1}$ and the anodic peak doublet and associated cathodic peak doublet observed for the layer prepared at $20 \mathrm{mV} \mathrm{s}^{-1}$. On the other hand, only a single broad anodic and cathodic peak corresponding to the $\alpha /$ $\gamma-\mathrm{Ni}$ (II/III) transition could be noted for the layers deposited at shorter timescales. The latter authors attributed this effect to dehydration within the layer which would be expected to increase for longer experimental timescales and would be reflected in the appearance of the set of $\beta / \beta$ redox peaks associated with the more anhydrous $\beta-\mathrm{Ni}(\mathrm{OH})_{2}$ phase of the oxide material. Furthermore, Lyons and coworkers ${ }^{167}$ found that the sweep rate can also affect the OER electrocatalytic properties of potentiodynamically deposited $\mathrm{Ni}(\mathrm{OH})_{2}$ films. In Fig. 6 it is clear that a lower OER onset potential and higher currents at a fixed OER potential were observed for the $\mathrm{Ni}(\mathrm{OH})_{2}$ film deposited at $20 \mathrm{mV} \mathrm{s}^{-1}$, indicating a significant improvement in the OER activity of this film in comparison with those prepared at $10 \mathrm{mV} \mathrm{s}^{-1}$ or $50 \mathrm{mV} \mathrm{s}^{-1}$.

However, among the various experimental parameters the electrode substrate seems to have the greatest influence on the electrocatalytic properties of the oxide/hydroxide films. El-Deab et $a l .{ }^{29}$ compared the OER electrocatalytic activity of $\gamma-\mathrm{MnOOH}$ nanorods deposited on $\mathrm{Au}, \mathrm{Pt}$ and glassy carbon (GC) substrates. They found that the lowest OER onset potential was obtained for a manganese oxide modified $\mathrm{Au}$ substrate. In a number of recent publications, Bell and coworkers ${ }^{25,27}$ have also highlighted the effect of the substrate on the electrocatalytic properties of $\mathrm{Ni}$ and Co oxides. In particular, they observed that the OER activity of a submonolayer of cobalt oxide decreases in the order $\mathrm{CoO}_{x} / \mathrm{Au}>\mathrm{CoO}_{x} / \mathrm{Pt}>\mathrm{CoO}_{x} / \mathrm{Pd}>\mathrm{CoO}_{x} /$ $\mathrm{Cu}>\mathrm{CoO}_{x} / \mathrm{Co}^{27}$ This trend was interpreted in terms of the electronegativities of the substrates. That is, the more electronegative metals, such as $\mathrm{Au}$, can facilitate the formation of higher Co oxidation states thereby improving the electrocatalytic properties of the oxide. Similarly, the latter authors found that a monolayer of nickel oxide deposited on $\mathrm{Au}$ exhibited greater electrocatalytic activity when compared with an equivalent layer deposited on a Pd substrate. ${ }^{25}$ 
Interestingly, in the case of very thin layers of nickel oxide deposited on Au substrates, Bell and coworkers ${ }^{25}$ detected the formation of a mixed $\mathrm{Ni}-\mathrm{Au}$ oxide on the substrate surface, suggesting that the substrate can also influence the composition of the oxide. Indeed, Lyons and co-workers ${ }^{167}$ have observed similar substrate effects for the redox characteristics of $\mathrm{Ni}(\mathrm{OH})_{2}$ films. The latter authors noted distinct differences in the voltammetric profiles of $\mathrm{Ni}(\mathrm{OH})_{2}$ films deposited on $\mathrm{Au}$, Pt and GC substrates. Representative cyclic voltammograms recorded for $\mathrm{Ni}(\mathrm{OH})_{2}$ deposits on each substrate are presented in Fig. 7. It is noteworthy that a clear distinction between the $\alpha / \gamma$ and $\beta / \beta$ redox transitions of $\mathrm{Ni}(\mathrm{OH})_{2}$ could be observed using the $\mathrm{Au}$ substrate, whereas this distinction was significantly less
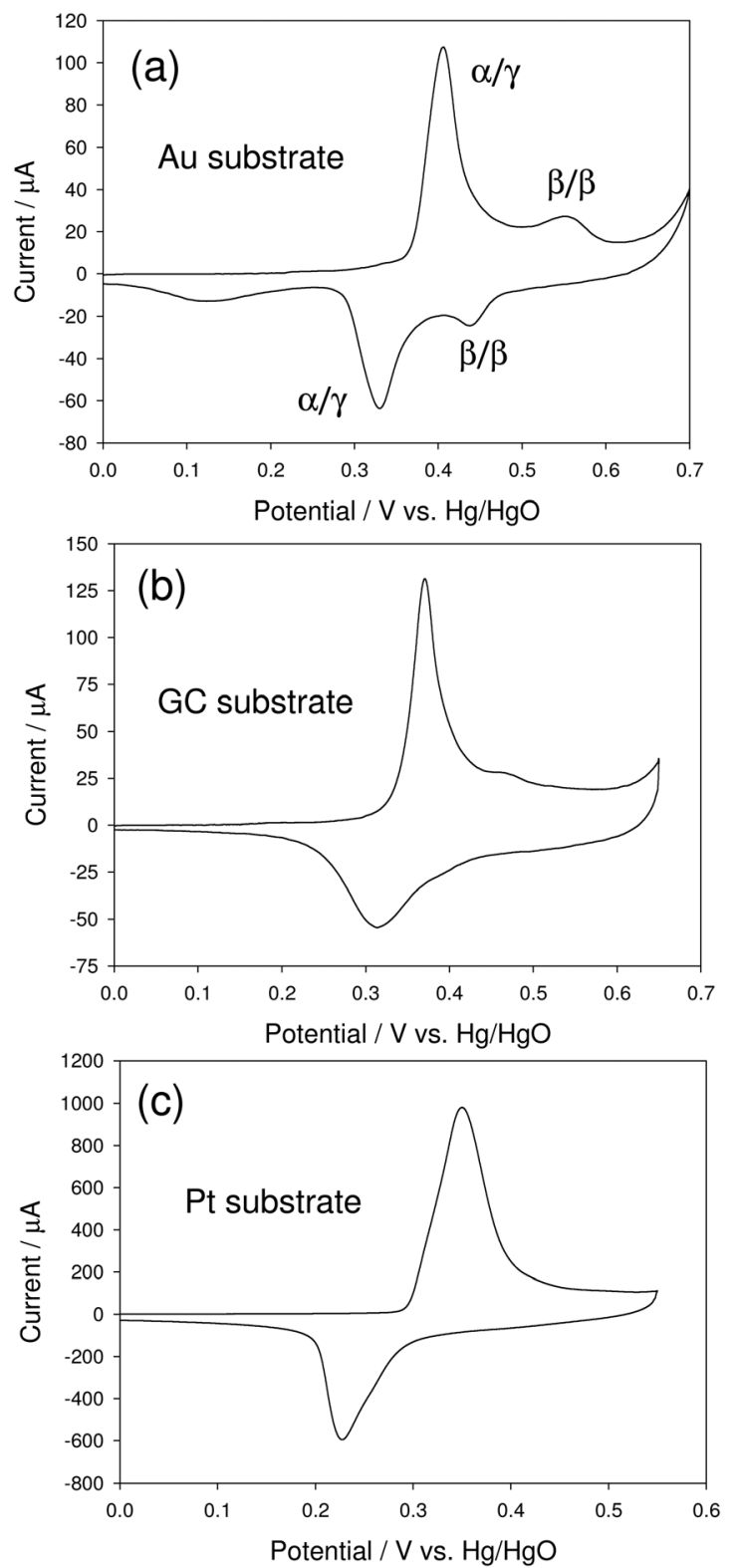

Fig. 7 Cyclic voltammograms recorded in $1.0 \mathrm{M} \mathrm{NaOH}$ at a sweep rate of $40 \mathrm{mV} \mathrm{s}^{-1}$ for (a) Au, (b) GC and (c) Pt electrodes modified with nickel hydroxide films deposited using 30 deposition cycles. well defined for the GC and Pt substrates. Clearly the composition of the oxide layer and/or the kinetics of the redox switching processes were affected by the nature of the substrate.

\section{Redox switching and acid/base properties of transition metal oxides: the Surfaquo group}

In general, the redox switching behaviour exhibited by transition metal oxides in contact with aqueous solutions is complex. Redox switching in this sense refers to the potential induced change in oxidation state of linked oxy-metal groups which constitute the metal oxide film. Consequently, the redox behaviour of transition metal oxides is highly influenced by both the nature of the oxide and the manner of its preparation. Depending on a range of factors including the composition and structure of the oxide, and the available oxidation states of the metal, various redox transitions may occur which give rise to the characteristic charge storage and supercapacitive behaviour associated with such films.

In view of this variety and complexity, it is important to point out a common feature of transition metal oxide redox chemistry. That is, surface oxy groups are known to hydrate or hydroxylate in aqueous solution. ${ }^{174,175}$ Metal oxide surfaces have a significant hydrophilic character when immersed in aqueous alkaline solutions. As the oxide interacts with water, solvent molecules can become bonded to the metal cations, which exhibit Lewis acidity, resulting in the transfer of a proton to a neighbouring oxygen site. In addition, undissociated water molecules may also be present. Hence, the surface oxy groups can become extensively hydrated or hydroxylated. Indeed, it has been noted that the chemical composition of the outer region of thermally prepared DSA type oxides, such as $\mathrm{RuO}_{2}$ and $\mathrm{IrO}_{2}$, in contact with aqueous acid and base solutions may not be dissimilar to the anodically generated hydrous oxide films prepared via potential cycling of the parent metal in the corresponding aqueous media, although the degree of hydration/ hydroxylation exhibited by the thermally prepared films may differ substantially from that of their electrochemically prepared analogs. ${ }^{176,177}$ These hydrated surface species, termed surfaquo groups, may undergo rapid redox transformations involving the simultaneous loss or gain of electrons, protons and hydroxide ions. ${ }^{12,178}$ In the case of the hydrous oxides the redox transition likely involves the entire film, whereas the redox reactions are confined to surface oxymetal groups in the case of the more compact thermal deposits. Thus, these redox reactions are often both time and potential dependent and can be readily monitored in real time using potential sweep voltammetry.

In the present section we focus on the interfacial redox chemistry of representative noble, $\mathrm{Ru}$ and $\mathrm{Ir}$, and non-noble, $\mathrm{Fe}$, Ni and Co, metal oxide materials. A brief overview of the redox characteristics of each material is provided with the intention of gaining a conceptual understanding of the oxide surface that facilitates the OER. Taking into account the surfaquo group concept, the redox behaviour of the various 
oxide materials is interpreted in terms of the redox chemistry of hydrated surface groups. In particular, we highlight how a study of the $\mathrm{pH}$ dependence of the redox potentials can aid in determining the nature of the surfaquo groups associated with each material.

\subsection{Interfacial redox chemistry of ruthenium and iridium based electrodes in alkaline solution}

The general aspects of the redox behaviour of ruthenium and iridium based thermally prepared oxide electrodes are discussed here. Typical voltammetric curves recorded in aqueous base for thermally prepared $\mathrm{RuO}_{2}$ coatings on a Ti substrate are presented in Fig. 8. There is now widespread agreement that the charge recorded during the course of voltammetric experiments for such oxide films is largely pseudocapacitive in nature. That is, the charge is mainly associated with interfacial redox processes. Distinct reversible peaks are noted at $c a .0 .45 \mathrm{~V}$ prior to the onset of active oxygen gas evolution for $\mathrm{RuO}_{2}$ based electrodes with two further sets of broad, less well defined peaks at lower potentials of $c a$. $0.2 \mathrm{~V}$ and $-0.3 \mathrm{~V}$ respectively. As can be observed from Fig. 8, most of the charge associated with these redox processes is distributed rather broadly over an extended potential range which signifies an energetic heterogeneity with regard to the redox active sites within the oxide surface. It is also clear that the integrated voltammetric charge recorded between the potential limits in Fig. 8 is relatively symmetric, indicating that a high degree of reversibility is exhibited by the interfacial redox processes.

Accordingly, it has been suggested that the voltammetric peaks observed for both $\mathrm{RuO}_{2}$ and $\mathrm{IrO}_{2}$ based oxide systems may be assigned to a series of redox transformations involving tightly bound surface oxymetal species. ${ }^{12,57,178}$ The specific nature of these redox active surface groups has been discussed by Lyons, Burke and coworkers. ${ }^{12,57,75,176}$ It was determined that only oxycations at certain sites, presumably sites of low coordination such as kink or ledge sites, are capable of participating in the redox reactions giving rise to the peaks

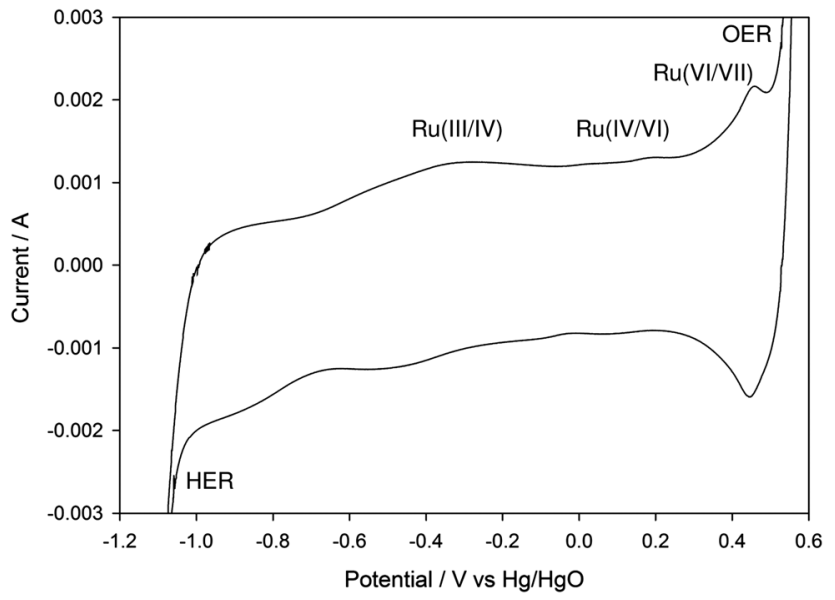

Fig. 8 Typical voltammogram recorded at $40 \mathrm{mV} \mathrm{s}^{-1}$ in $1.0 \mathrm{M} \mathrm{NaOH}$ for a thermally prepared $\mathrm{RuO}_{2}$ coated Ti substrate. observed in the voltammetric response. ${ }^{12,176}$ Bearing in mind our previous discussion on the hydrophilic nature of surface oxy groups, the important factor here seems to be the ability of the latter type of cations to extensively coordinate water molecules. Inactive ruthenium or iridium species would exhibit a higher degree of oxygen bridging type coordination and would most likely exist along terrace sites. These ideas emphasising the important role of hydration in determining the difference between active and inactive surface bonded groups led to the designation of the former as surfaquo groups. Although the structure of such active surfaquo groups is unknown, it is very likely that the linkage to the surface involves one or more oxygen bridges to generate the following type of hydrous species,

$$
\begin{gathered}
(-\mathrm{O}-)_{2} \mathrm{RuO}_{2}\left(\mathrm{OH}_{2}\right)_{2} \leftrightarrow(-\mathrm{O}-)_{2} \mathrm{RuO}(\mathrm{OH})_{2}\left(\mathrm{OH}_{2}\right) \\
(-\mathrm{O}-)_{2} \mathrm{RuO}_{2}\left(\mathrm{OH}_{2}\right)_{2} \leftrightarrow(-\mathrm{O}-)_{2} \mathrm{Ru}(\mathrm{OH})_{4}
\end{gathered}
$$

with the bridging oxygens being represented by -O-. The latter structures represent $\mathrm{Ru}(\mathrm{vI})$ surfaquo species and such equilibria between hydrated oxides, oxyhydroxides and hydroxides are well-known for hydrated oxides.

The redox behaviour of such surface immobilized oxymetal groups is assumed to involve the simultaneous loss or gain of protons and electrons. ${ }^{57,75}$ It is well established that thermally prepared $\mathrm{RuO}_{2}$ films exhibit three distinct redox processes involving $\mathrm{Ru}(\mathrm{III} / \mathrm{Iv}), \mathrm{Ru}(\mathrm{Iv} / \mathrm{VI})$ and $\mathrm{Ru}(\mathrm{VI} / \mathrm{VII})$ surface redox transitions. Thus, the various $\mathrm{RuO}_{2}$ redox transformations may be represented respectively by the following equations, ${ }^{57,75}$

$$
\begin{aligned}
(-\mathrm{O}-)_{2} \mathrm{Ru}(\mathrm{OH})\left(\mathrm{OH}_{2}\right)_{3} & \rightarrow(-\mathrm{O}-)_{2} \mathrm{Ru}(\mathrm{OH})_{2}\left(\mathrm{OH}_{2}\right)_{2}+\mathrm{H}^{+}+\mathrm{e}^{-} \\
(-\mathrm{O}-)_{2} \mathrm{Ru}(\mathrm{OH})_{2}\left(\mathrm{OH}_{2}\right)_{2} & \rightarrow(-\mathrm{O}-)_{2} \mathrm{Ru}(\mathrm{OH})_{4}+2 \mathrm{H}^{+}+2 \mathrm{e}^{-} \\
(-\mathrm{O}-)_{2} \mathrm{Ru}(\mathrm{OH})_{4} & \rightarrow(-\mathrm{O}-)_{2} \mathrm{RuO}(\mathrm{OH})_{3}+\mathrm{H}^{+}+\mathrm{e}^{-}
\end{aligned}
$$

The standard potentials of which, quoted against the reversible hydrogen electrode (RHE), are typically $0.45 \mathrm{~V}, 1.0 \mathrm{~V}$ and $1.4 \mathrm{~V}$ respectively. It has been observed that the latter transition which occurs just prior to the onset of oxygen evolution is most marked in base. On the other hand, the redox chemistry of $\mathrm{IrO}_{2}$ involves a major charge storage Ir(III/Iv) transition which can be represented by the following,

$(-\mathrm{O}-)_{2} \mathrm{Ir}(\mathrm{OH})\left(\mathrm{OH}_{2}\right)_{3} \rightarrow(-\mathrm{O}-)_{2} \operatorname{Ir}(\mathrm{OH})_{2}\left(\mathrm{OH}_{2}\right)_{2}+\mathrm{H}^{+}+\mathrm{e}^{-}$

and an $\operatorname{Ir}(\mathrm{IV} / \mathrm{VI})$ transition at more elevated anodic potentials prior to the onset of active oxygen evolution,

$$
(-\mathrm{O}-)_{2} \operatorname{Ir}(\mathrm{OH})_{2}\left(\mathrm{OH}_{2}\right)_{2} \rightarrow(-\mathrm{O}-)_{2} \mathrm{Ir}(\mathrm{OH})_{4}+2 \mathrm{H}^{+}+2 \mathrm{e}^{-}
$$

In this way the redox behaviour of $\mathrm{RuO}_{2}$ and $\mathrm{IrO}_{2}$ films can be rationalised in terms of the redox chemistry of the surfaquo groups.

The surface redox chemistry of metal oxides may be further understood by examining the manner in which the peak potentials derived from the cyclic voltammograms vary in magnitude with changes in solution $\mathrm{pH}$. While the latter expressions explain the high degree of reversibility exhibited by the $\mathrm{RuO}_{2}$ and $\mathrm{IrO}_{2}$ redox peaks, they do not provide a rationale for the proposed acid/base 
character of the metal oxides. ${ }^{12}$ Burke and Lyons ${ }^{17,45}$ have shown that, for an ideal oxide electrode system in aqueous solution at $25{ }^{\circ} \mathrm{C}$, the potential decreases with increasing $\mathrm{pH}$ by ca. $59 \mathrm{mV}$ per $\mathrm{pH}$ unit with respect to a $\mathrm{pH}$ independent reference electrode such as the normal hydrogen electrode (NHE) or the saturated calomel electrode (SCE). This type of potential-pH shift is referred to as a Nernstian shift, since it is predicted by the Nernst equation, and is typified by the reversible hydrogen electrode (RHE) and the $\mathrm{Hg}-\mathrm{HgO}$ system, which is frequently the reference electrode of choice in alkaline solution. In this respect, it should be noted that no potential $\mathrm{pH}$ shift will be observed if the reference electrode is $\mathrm{pH}$ dependent since the potential of this type of electrode also exhibits a Nernstian shift. On the other hand, so called superNernstian shifts, where the potential-pH dependence differs significantly from the expected $59 \mathrm{mV}$ per $\mathrm{pH}$ unit predicted by a Nernstian analysis, are often observed for the various metal oxide systems. ${ }^{17,45}$ It has been shown that for pure $\mathrm{RuO}_{2}$ electrodes the shifts in peak potential with changes in solution $\mathrm{pH}$ for the lower redox transitions are typically $15 \mathrm{mV}$ per $\mathrm{pH}$ unit $v s$. a pH dependent reference electrode such as the RHE or about $75 \mathrm{mV}$ per $\mathrm{pH}$ unit for a $\mathrm{pH}$ independent reference electrode such as the SCE, ${ }^{178}$ whereas even larger shifts have been observed for the hydrous oxide films (as discussed in Section $3.2 \& 3.3$ ).

This type of super-Nernstian behaviour has been discussed in detail by Burke and Lyons ${ }^{175-177}$ and is usually interpreted in terms of hydrolysis effects. That is, the effect of the loss of protons from water molecules co-ordinated to the metal cation. Transition metal aquocations tend to be acidic in aqueous media and are prone to hydrolyse according to the following equation, ${ }^{179}$

$$
\left[\mathrm{M}\left(\mathrm{OH}_{2}\right)_{x}\right]^{n+} \rightarrow\left[\mathrm{M}\left(\mathrm{OH}_{2}\right)_{x-1}(\mathrm{OH})\right]^{(n-1)+}+\mathrm{H}_{\mathrm{aq}}{ }^{+}
$$

In particular, Burgess ${ }^{180}$ has noted that there is an increase in the acidity of the latter metal complexes with increasing charge on the metal cation. Therefore, by analogy with the solution phase, the hydrolysis effect for metal oxide surfaces is assumed to be greater for the oxidised state of the redox couple where the greater charge density on the cation facilitates proton loss from coordinated water molecules. The extent of this effect is apparently lower in the case of thermally prepared films when compared with the hydrous oxides, as evidenced by their lower super-Nernstian shifts. The bonding of the oxycations to the rigid, anhydrous, highly conducting $\mathrm{RuO}_{2}$ lattice evidently affects the hydrolysis process, although the precise manner by which this occurs has yet to be established unambiguously. Given the importance of the charge on the metal cation, it is possible that the resulting electric field around the hydrolysis site is slightly reduced by interactions with the highly mobile electrons in the compact solid. Alternative factors which may be of importance are: (i) a more limited access, and therefore influence, of $\mathrm{OH}^{-}$ions to the surface bonded species, (ii) the two bridging oxygens in the surface groups are not protonated at any stage making the remaining hydrogen atoms less likely to be removed and (iii) some of the remaining hydrogen atoms may be partly stabilized by interactions either with the surface or other nearby surface groups.

The mathematical treatment of such super-Nernstian potential-pH behaviour, where the redox switching of the oxide layer is complicated by additional hydrolysis effects, has been described by Lyons and coworkers. ${ }^{17,175-177}$ Using a simple thermodynamic argument, the variation in voltammetric peak potential with $\mathrm{pH}$ may be predicted to be,

$$
\frac{\mathrm{d} E}{\mathrm{dpH}}=-\frac{2.303 R T}{F}(r-q)=-0.059(r-q) V / \mathrm{pH}
$$

where $r$ and $q$ denote the number of hydroxide ions complexed to the metal cation in the oxidised and reduced form respectively. Thus, a zero potential shift with respect to a $\mathrm{pH}$ dependent reference electrode implies that both the reactant and the product possess the same net charge and typical Nernstian behaviour is observed. Alternatively, a positive potential shift with $\mathrm{pH}$ is indicative of an oxidised state that is more positive than the reduced state, whereas the converse is true in the case of an observed negative potential/pH shift. For most surface groups $r$ and $q$ cannot be determined explicitly, instead the quantity $(r-q)$ can be interpreted as the ratio of protons gained or hydroxide ions lost per electron gained in the reduction of the surfaquo group. Consequently, the experimentally observed $5 / 4(2.303 R T / F) \mathrm{V}$ or $0.074 \mathrm{~V}$ potential-pH shift observed for the $\mathrm{Ru}(\mathrm{III} / \mathrm{IV})$ and $\mathrm{Ru}(\mathrm{Iv} / \mathrm{vI})$ transitions implies that $r-q=1.25$ which can be rationalised by the following reaction schemes,

$$
\begin{aligned}
(-\mathrm{O}-)_{2} \mathrm{Ru}(\mathrm{OH})\left(\mathrm{OH}_{2}\right)_{3} \rightarrow & (-\mathrm{O}-)_{2} \mathrm{Ru}^{0.25-}(\mathrm{OH})_{2.25}\left(\mathrm{OH}_{2}\right)_{1.75} \\
+1.25 \mathrm{H}^{+} & +\mathrm{e}^{-} \\
(-\mathrm{O}-)_{2} \mathrm{Ru}^{0.25-}(\mathrm{OH})_{2.25}\left(\mathrm{OH}_{2}\right)_{1.75} \rightarrow & (-\mathrm{O}-)_{2} \mathrm{RuO}_{0.75}(\mathrm{OH})_{3.25} \\
+ & 2.5 \mathrm{H}^{+}+2 \mathrm{e}^{-}
\end{aligned}
$$

The non-integral charge value could possibly arise as a mean value in a polymeric or interlinked system of surfaquo groups. Indeed, the importance of such hydroxy complexes as possible intermediates in the formation of polynuclear species held together by hydroxyl or oxy bridges has been outlined by Lyons and co-workers ${ }^{17,18,45}$ in relation to the hydrous oxide materials. The latter structural links act as the glue by which the extended microdispersed hydrous oxide structures are held together.

Another important feature relating to the redox switching behaviour of metal oxide films is the integrated voltammetric charge $Q^{*}$. As noted previously, the voltammetric charge arises from the surface redox transitions outlined above and thus, is a very useful parameter to facilitate characterization of the interfacial properties of metal oxide films in aqueous solutions. In particular, the value of $Q^{*}$ determined between pre-defined limits has been shown to be a relative measure of the active surface area of the oxide film. ${ }^{48,181}$ In this sense, Lyons and Burke $^{57}$ have noted that cyclic voltammetry provides a useful technique for obtaining an estimate of the active surface-group 
coverage $\Theta$. The surface-group coverage $\Theta$ may be regarded as the ratio of the surface area occupied by a surface group $S_{\mathrm{G}}$ to the total (or real) surface area $S_{\mathrm{T}}$,

$$
\Theta=S_{\mathrm{G}} / S_{\mathrm{T}}
$$

It has been shown for various $\mathrm{RuO}_{2}$ based oxide coatings that the voltammetric charge recorded under potential cycling conditions is a linear function of surface area. ${ }^{182}$ Accordingly, substitution for the quantity $S_{\mathrm{T}}$ is possible using the relationship,

$$
Q^{*}=Q_{\mathrm{T}}=k S_{\mathrm{T}} X_{\mathrm{RuO}_{2}}
$$

where $k$ is a constant of proportionality and $X_{\mathrm{RuO}_{2}}$ denotes the mole fraction of $\mathrm{RuO}_{2}$ in the surface layer. In order to obtain a similar relation for $S_{\mathrm{G}}$ it may be assumed, to a first approximation, that the octahedrally coordinated surface group is of roughly circular geometry. Now, if there are $N_{\mathrm{G}}$ such groups on the surface and it is assumed that all active groups react at the redox potential then,

$$
S_{\mathrm{G}}=N_{\mathrm{G}} \pi R^{2}=\frac{Q_{\mathrm{p}}}{e} \pi R^{2}
$$

where $R$ denotes the mean radius of the surface species, $Q_{\mathrm{p}}$ is the peak charge density and $e$ is the electronic charge. Hence, substitution of eqn (20) and (21) into eqn (19) yields the following expression for the surface coverage $\Theta$,

$$
\Theta=\frac{k Q_{\mathrm{p}} \pi R^{2}}{e Q_{\mathrm{T}}} X_{\mathrm{RuO}_{2}}
$$

It should be noted that the quantities $Q_{\mathrm{p}}$ and $Q_{\mathrm{T}}$ may be obtained from cyclic voltammograms run between set limits and under given experimental conditions. For a pure $\mathrm{RuO}_{2}$ film in $6.0 \mathrm{M} \mathrm{NaOH}$ at $80{ }^{\circ} \mathrm{C}$, Lyons and Burke ${ }^{57}$ report typical values of $Q_{\mathrm{p}}=0.022 \mathrm{C}$ for the $\mathrm{Ru}(\mathrm{vI} / \mathrm{vII})$ redox peak and $Q_{\mathrm{T}}=0.23 \mathrm{C}$. In addition, it has been shown that $k=1.6 \times 10^{-2} \mathrm{C} \mathrm{cm}^{-2}$ $\left(\mathrm{mol} \% \mathrm{RuO}_{2}\right)^{-1} \cdot{ }^{182}$ Using values of 0.67 and $1.32 \AA$ for the ionic radii of the $\mathrm{Ru}^{4+}$ and $\mathrm{O}^{2-}$ ions respectively, ${ }^{183} R$ can be approximated as $3.3 \times 10^{-10} \mathrm{~m}$. Hence, a value of $c a .0 .34 \mathrm{~nm}^{2}$ is obtained for the area occupied by a surface group, which compares well with the commonly accepted value of $c a .0 .16 \mathrm{~nm}^{2}$ for a nitrogen molecule. Finally, if it is assumed that $e=1.6 \times 10^{-19} \mathrm{C}$ then substitution of these values into eqn (22) yields a value of 0.3 for the active surface group coverage. Interestingly, as noted by Lyons and Burke, ${ }^{57}$ this value is in good agreement with coverage values reported by Bockris et al. ${ }^{184,185}$ for perovskite based oxide systems, thereby highlighting the potential usefulness of this rather simple analysis.

\subsection{Interfacial redox chemistry of iron based electrodes in alkaline solution}

Typical voltammetric profiles recorded for a polycrystalline Fe electrode in $1.0 \mathrm{M} \mathrm{NaOH}$ are presented in Fig. 9. In the initial stages of oxidation, the voltammetric profiles exhibit quite an amount of fine structure. As shown in Fig. 9a, four well defined anodic peaks (A1-A4) and two cathodic peaks (C1, C2) are usually observed. In addition, it has been noted that the general
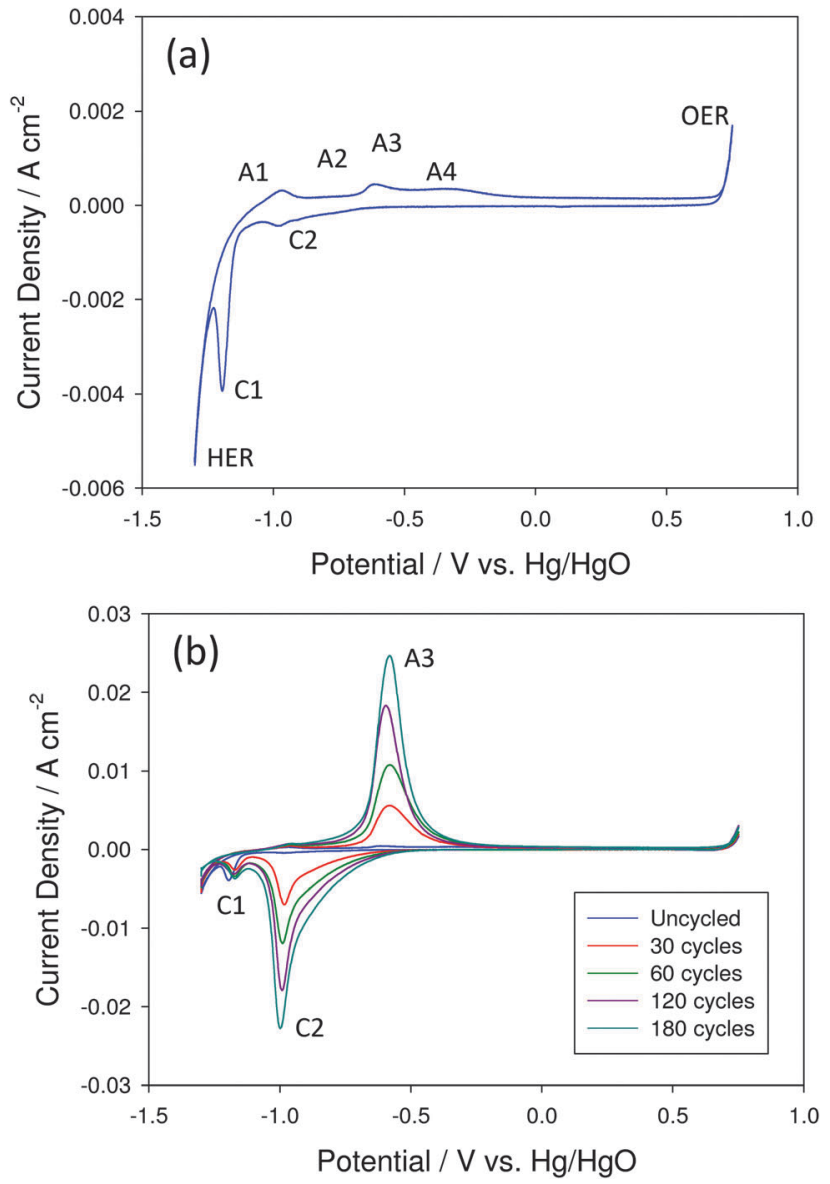

Fig. 9 Cyclic voltammograms recorded in $1.0 \mathrm{M} \mathrm{NaOH}$ at $40 \mathrm{mV} \mathrm{s}^{-1}$ for (a) a polycrystalline Fe electrode and (b) a series of hydrous oxide coated Fe electrodes prepared using various numbers of growth cycles between $-1.3 \mathrm{~V}$ and $0.75 \mathrm{~V}$ at a sweep rate of $400 \mathrm{mV} \mathrm{s}^{-1}$.

features of the voltammetric response remain unchanged, even if the concentration of base is increased. ${ }^{84}$ These various features, reflecting surface redox processes involving surface bound oxy iron species, have been assigned by a number of authors in the literature. ${ }^{186-195}$ Our current viewpoint is primarily informed by the work of Burke and Lyons. ${ }^{84}$

Peak A1 is most probably due to the formation of a layer of adsorbed hydroxy species,

$$
\mathrm{Fe}+\mathrm{OH}^{-} \rightarrow \mathrm{FeOH}_{\mathrm{ads}}+\mathrm{e}^{-}
$$

combined with the electrochemical displacement of adsorbed hydrogen,

$$
\mathrm{FeH}_{\mathrm{ads}} \rightarrow \mathrm{Fe}+\mathrm{H}^{+}+\mathrm{e}^{-}
$$

Peak A2 may then represent the conversion of both Fe and $\mathrm{FeOH}_{\text {ads }}$ to a thin film of $\mathrm{Fe}(\mathrm{II})$ hydroxide or oxide according to,

$$
\begin{gathered}
\mathrm{FeOH}_{\mathrm{ads}}+\mathrm{OH}^{-} \rightarrow \mathrm{Fe}(\mathrm{OH})_{2}+\mathrm{e}^{-} \\
\mathrm{FeOH}_{\mathrm{ads}}+\mathrm{OH}^{-} \rightarrow \mathrm{FeO}_{\mathrm{ads}}+\mathrm{H}_{2} \mathrm{O}+\mathrm{e}^{-}
\end{gathered}
$$

However, due to a variety of other possible reactions, the overall interfacial reaction resulting in $\mathrm{Fe}(\mathrm{II})$ film formation 
may be more complex than that outlined above. For instance hydroxylation reactions such as,

$$
\mathrm{FeO}+\mathrm{H}_{2} \mathrm{O} \rightarrow \mathrm{Fe}(\mathrm{OH})_{2}
$$

will result in the conversion of oxy to hydroxy species in the outer region of the surface layer. Also, place exchange processes can result in an increase in the thickness of the surface layer. ${ }^{196-199}$ The latter type of growth process has been proposed to involve a rapid place exchange step followed by a rate determining Temkin discharge of $\mathrm{OH}^{-}$ions onto sites in which a surface iron atom is already attached to a hydroxyl group displaced into the first layer beneath the surface,

$$
\begin{gathered}
\mathrm{FeOH}_{\mathrm{ads}} \rightarrow \mathrm{HOFe} . . \text { (Fast) } \\
\mathrm{HOFe}+\mathrm{OH}^{-} \rightarrow \mathrm{HOFeOH}_{\text {ads }} \ldots \text { (Slow) } \\
\mathrm{HOFeOH} \text { ads } \rightarrow \mathrm{Fe}(\mathrm{OH})_{2} \rightarrow \mathrm{FeO}+\mathrm{H}_{2} \mathrm{O}
\end{gathered}
$$

For a non-noble metal such as iron, the aforementioned surface processes are likely to be accompanied by film thickening even at quite low potentials. Furthermore, it has been noted that active dissolution to form soluble oxy iron species may occur according to, ${ }^{200,201}$

$$
\begin{gathered}
\mathrm{FeO}+\mathrm{OH}^{-} \rightarrow \mathrm{HFeO}_{2}{ }^{-} \\
\mathrm{HFeO}_{2}{ }^{-}+\mathrm{OH}^{-} \rightarrow \mathrm{FeO}_{2}{ }^{2-}+\mathrm{H}_{2} \mathrm{O}
\end{gathered}
$$

As a result, a deposit of gelatinous ferrous oxide $\mathrm{Fe}(\mathrm{OH})_{2}$ which is weakly bound to the metal can be formed by the subsequent hydrolysis of the $\mathrm{HFeO}_{2}{ }^{-}$ion,

$$
\mathrm{HFeO}_{2}{ }^{-}+\mathrm{H}_{2} \mathrm{O} \rightarrow \mathrm{Fe}(\mathrm{OH})_{2}+\mathrm{OH}^{-}
$$

In simple terms, peak A3 and its cathodic counterpart $\mathrm{C} 2$ are attributed to the following $\mathrm{Fe}(\mathrm{II}) / \mathrm{Fe}$ (III) redox transformation,

$$
\mathrm{Fe}(\mathrm{OH})_{2}+\mathrm{OH}^{-} \rightarrow \mathrm{FeOOH}+\mathrm{H}_{2} \mathrm{O}+\mathrm{e}^{-}
$$

which involves an electrochromic colour change from transparent to yellow/green as the oxidation state changes from $\mathrm{Fe}(\mathrm{II})$ to $\mathrm{Fe}(\mathrm{III})$. However, it has been noted previously that the A3/C2 peaks exhibit the usual characteristics of a hydrated or hyperextended oxide. Lyons and coworkers ${ }^{84,96,98}$ have reported super-Nernstian potential-pH shifts for peaks $\mathrm{A} 3 / \mathrm{C} 2$ of the order of $\mathrm{d} E / \mathrm{dpH}=-2.303(3 R T / 2 F)=-0.088 \mathrm{~V}$ per $\mathrm{pH}$ unit at $T=298 \mathrm{~K}$, indicating that the oxide acquires a net negative charge in the oxidised state relative to the reduced state. Since traces of this peak appear even during the first sweep of a fresh, previously unused electrode, it is possible that at the outer regions of the oxide film the hydroxylation process outlined in eqn (27) is succeeded by a further stage which involves coordination of additional $\mathrm{H}_{2} \mathrm{O}$ and $\mathrm{OH}^{-}$species. Indeed, this concept of a hydrated outer oxide layer is well established for Fe electrodes. Early ellipsometric and spectroscopic studies performed by Bockris and coworkers ${ }^{202,203}$ and O'Grady ${ }^{204}$ pointed to the presence of bound water in the passive layer formed on polycrystalline iron. In particular, these authors suggested a hydrated polymeric oxide model for the passive oxide layer. Furthermore, it can be seen from Fig. 9b that the
A3/C2 peaks are the only peaks to display a significant enhancement upon potential cycling. Hence the latter peak combination is attributed to an $\mathrm{Fe}(\mathrm{II}) / \mathrm{Fe}(\mathrm{III})$ redox transition in a polymer microdispersed hydrous oxide layer, formed initially by hydration of the outer regions of the $\mathrm{Fe}(\mathrm{OH})_{2}$ or $\mathrm{FeO}$ film.

In recent years, the redox switching and interfacial behaviour of such hydrous oxide modified electrodes have been examined using Probe Beam Deflection (PBD) and Electrochemical Quartz Crystal Microbalance (EQCM). These techniques have proved very useful in the real time study of coupled electron and ion transport processes in these films and have been described by Lyons ${ }^{205}$ and by Oyama and Ohsaka. ${ }^{206}$ In relation to the hydrous oxide layers prepared on iridium electrodes, Kotz and co-workers ${ }^{207}$ determined that protons are ejected from the film during the course of oxidation in acid solution, whereas hydroxide ions and charge compensating counterions are consumed for the same process in base. In light of this, it is reasonable to suppose that similar processes are also involved in the redox switching reaction of iron oxyhydroxide layers. Accordingly, by analogy with a scheme produced by Burke and Whelan ${ }^{176}$ for redox switching of iridium oxide films, it has been proposed that the main charge storage reaction represented by peaks A3/C2 may be better described by, ${ }^{84}$

$$
\begin{aligned}
{\left[\mathrm{Fe}_{2}(\mathrm{OH})_{6}\left(\mathrm{OH}_{2}\right)_{3}\right]_{n}{ }^{2-} } & +3 n \mathrm{OH}^{-} \rightarrow\left[\mathrm{Fe}_{2} \mathrm{O}_{3}(\mathrm{OH})_{3}\left(\mathrm{OH}_{2}\right)_{3}\right]_{n}{ }^{3-} \\
& +3 n \mathrm{H}_{2} \mathrm{O}+2 n \mathrm{e}^{-}
\end{aligned}
$$

with charge neutrality being maintained by the movement of charge compensating cations such as $\mathrm{Na}^{+}$in the case of Fig. 9. Importantly, it can be shown, following a similar treatment to that outlined in Section 3.1, that the ratio of hydroxide ions to electrons in the latter expression will yield the experimentally observed $\mathrm{pH}$ dependence. Hence, one has a rapid topotactic reaction involving hydroxide ion and counterion ingress and solvent egress at the oxide/solution interface, and electron injection at the metal/oxide interface.

In this way, the hydrous oxide film may be regarded as surface bound polynuclear species held together by hydroxyl or oxy bridges. It is noteworthy that the film has been described in terms of linked surfaquo groups which are arranged in a dispersed three dimensional structure with each group being electrocatalytically active. ${ }^{17}$ This is an important consideration when a model for the OER is being developed. Indeed, the surfaquo groups are specifically implicated as the catalytic centres in the hydrous oxide layers. ${ }^{96,98}$ The latter assertion being supported by the $\mathrm{pH}$ dependence of the OER at hydrous iron oxide covered electrodes. In Fig. 10, E-pH plots generated for peak A3 and the OER onset potential are presented for comparison. As stated previously, the redox peaks associated with the hydrous layer exhibit a super-Nernstian $E-\mathrm{pH}$ dependence, here A3 shifts negatively by $c a$. $0.1 \mathrm{~V}$ per $\mathrm{pH}$ unit. Significantly, the OER onset potential exhibits an $E-\mathrm{pH}$ shift of similar magnitude to peak A3, ca. $-0.093 \mathrm{~V}$ per $\mathrm{pH}$ unit, suggesting that the anionic surfaquo groups of the hydrous layer actively partake in oxygen evolution at hydrous oxide covered Fe electrodes. 


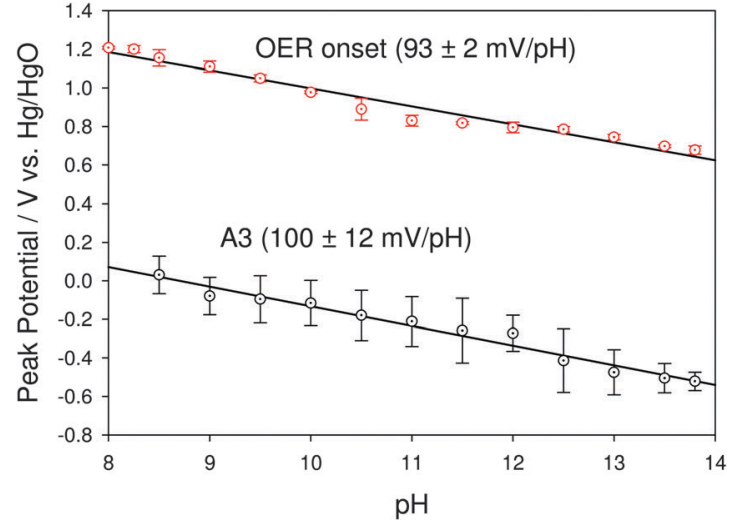

Fig. 10 The A3 peak potential and the OER onset potential for a typical hydrous iron oxide covered electrode plotted as a function of the solution $\mathrm{pH}$. Error bars representing $2 \sigma$ are also shown.

That said, it is important to emphasise that the formulae presented above should only be considered as a general guide. Other forms of $\mathrm{Fe}(\mathrm{II})$ and $\mathrm{Fe}(\mathrm{III})$ hydrated oxides are also possible and in fact mixtures such as hydrated $\mathrm{Fe}_{3} \mathrm{O}_{4}$ are quite likely. Indeed, the slightly irreversible nature of the main charge storage peaks, as evidenced by the difference in potential between $\mathrm{A} 3$ and $\mathrm{C} 2$, is more akin to the behavior exhibited by manganese rather than that of iridium and rhodium. In this sense, mixed oxide or hydroxide intermediate formation analogous to $\mathrm{Mn}_{3} \mathrm{O}_{4}$, or $\mathrm{Mn}(\mathrm{OH})_{3}$ may also be involved. ${ }^{208}$ Peak A4 is also related to an $\mathrm{Fe}$ (II)/Fe(III) redox transition. However, at this higher potential it is the inner more amorphous region of the Fe(II) species that is oxidised. Thus, the upper anodic peak A4 is probably due to an $\mathrm{Fe}(\mathrm{II} / \mathrm{Fe}$ (III) redox transition in the anhydrous compact oxide layer of $\mathrm{FeO}$ or $\mathrm{Fe}(\mathrm{OH})_{2}$ at the metal surface, leading to the formation of such species as $\gamma-\mathrm{Fe}_{2} \mathrm{O}_{3}$, $\mathrm{Fe}_{3} \mathrm{O}_{4}$ or $\mathrm{FeOOH}$.

The cathodic peak $\mathrm{C} 1$ corresponds to the reduction of the compact, anhydrous inner layer according to the following reaction,

$\mathrm{FeO} \cdot \mathrm{FeOOH}+\mathrm{H}_{2} \mathrm{O}+3 \mathrm{e}^{-} \rightarrow \mathrm{Fe}+\mathrm{FeO}_{2}{ }^{2-}+\mathrm{H}_{2} \mathrm{O}+\mathrm{OH}^{-}$

A useful aspect of this reaction is that the charge capacity of peak $\mathrm{C} 1$ can be used to estimate the extent of the compact layer growth. In this respect, it is to be noted from Fig. $9 \mathrm{~b}$ that the compact layer does not continue to grow to any great extent with increasing number of potential cycles.

\subsection{Interfacial redox chemistry of nickel based electrodes in alkaline solution}

The redox behaviour of nickel hydroxide and oxyhydroxide based electrodes is one of the most extensively studied systems in electrochemistry. This is due primarily to the practical utilisation of these electrodes in several technologically important areas including alkaline battery and electrolysis cells. However, progress in understanding the nature of the redox reactions at these materials in aqueous solution has been slow.
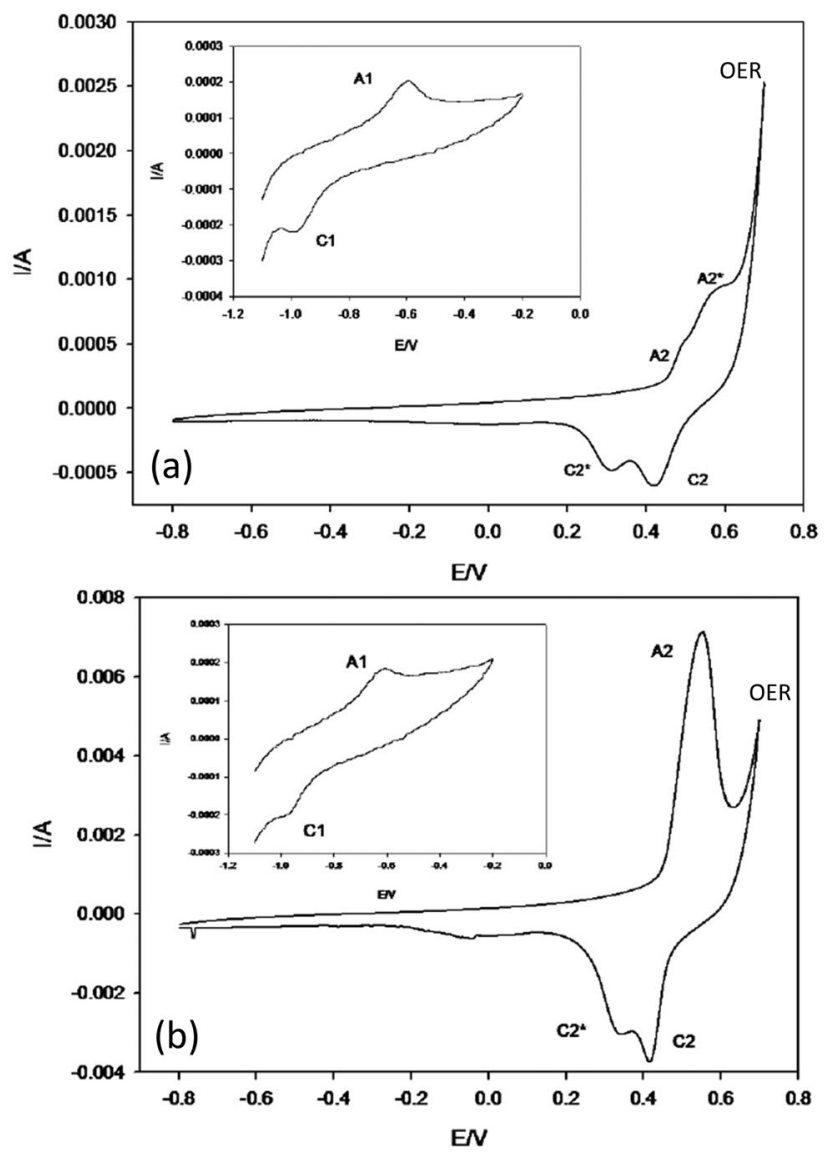

Fig. 11 Cyclic voltammograms recorded in $1.0 \mathrm{M} \mathrm{NaOH}$ at $40 \mathrm{mV} \mathrm{s}^{-1}$ for (a) a polycrystalline $\mathrm{Ni}$ electrode and (b) a hydrous oxide coated $\mathrm{Ni}$ electrode prepared using 300 growth cycles at $150 \mathrm{mV} \mathrm{s}^{-1}$ between $-1.45 \mathrm{~V}$ and $0.65 \mathrm{~V} v \mathrm{vs} . \mathrm{Hg} / \mathrm{HgO}$. The insets show the effect of reversing the potential sweep at $-0.2 \mathrm{~V}$, i.e. below the main charge storage peaks.

As noted by $\mathrm{McBreen}^{209}$ in his review on the electrochemistry of nickel hydroxides, the complexity of these reactions along with their variability with respect to factors such as electrode preparation and history has made normally routine analysis significantly more complicated. In the present discussion we briefly outline the current understanding of the redox chemistry of nickel and its oxides as it relates to electrochemically generated hydrous and bulk nickel oxide electrodes.

The voltammetric behaviour of polycrystalline Ni electrodes has been studied by a number of authors including Seghiouer and coworkers, ${ }^{210}$ Burke et al. ${ }^{177,211}$ Visscher and Barendrecht, ${ }^{212}$ de Souza et al. ${ }^{213}$ and Simpraga and Conway ${ }^{214}$ to name but a few. Typical voltammetric profiles in $1.0 \mathrm{M} \mathrm{NaOH}$ for a polycrystalline Ni electrode and a polycrystalline Ni electrode which has been subjected to a repetitive potential cycling regime are presented in Fig. 11. It is almost universally agreed that the lower anodic peak observed in the potential region between $-0.6 \mathrm{~V}$ and $-0.8 \mathrm{~V}$ is principally associated with the oxidation of metallic Ni to Ni(II) species, although there has been some disagreement about the nature of the $\mathrm{Ni}(\mathrm{II})$ oxide species formed at this potential. For example, Makrides ${ }^{215}$ proposed that $\mathrm{NiO}$ and $\mathrm{Ni}(\mathrm{OH})_{2}$ were produced, while Okuyama et al. ${ }^{216}$ 
suggested that the oxide film in this potential region was nonstoichiometric, consisting of $\mathrm{NiO}$ and $\mathrm{Ni}_{3} \mathrm{O}_{4}$. By analogy with the situation outlined for $\mathrm{Fe}$ in base the following sequence of reactions can be visualised at low potentials, ${ }^{91,217}$

$$
\begin{gathered}
\mathrm{Ni}+\mathrm{OH}^{-} \rightarrow \mathrm{NiOH}_{\mathrm{ads}}+\mathrm{e}^{-} \\
\mathrm{NiOH}_{\mathrm{ads}}+\mathrm{OH}^{-} \rightarrow \mathrm{Ni}(\mathrm{OH})_{2}+\mathrm{e}^{-} \\
\mathrm{NiOH}_{\mathrm{ads}}+\mathrm{OH}^{-} \rightarrow \mathrm{NiO}_{\mathrm{ads}}+\mathrm{H}_{2} \mathrm{O}+\mathrm{e}^{-}
\end{gathered}
$$

However, it should be noted that the overall interfacial reaction resulting in $\mathrm{Ni}(\mathrm{II})$ film formation will likely be complicated by surface hydroxylation reactions eqn (27) and place exchange film thickening reactions eqn (28-30) similar to those discussed for Fe above. Indeed, the complex nature of the film formed at these low potentials has been emphasised by Burke and Twomey. ${ }^{177,211}$ Based on the variation of the voltammetric peak potential with changes in $\mathrm{pH}$ in alkaline solution, $c a .13 \mathrm{mV}$ per $\mathrm{pH}$ unit $v s$. a $\mathrm{pH}$ independent reference electrode, they proposed that both oxidation and hydrolysis processes were operative in this region leading to the formation of a species with anionic character. For the purposes of rationalization this species was tentatively assigned the formula $\left[\mathrm{Ni}(\mathrm{OH})_{2.4}\right]^{0.4-}$. Hence, in very simple terms the net anodic reaction corresponding to peak $\mathrm{A} 1$ can be represented as,

$$
\mathrm{Ni}+2.4 \mathrm{OH}^{-} \rightarrow \mathrm{Ni}(\mathrm{OH})_{2.4}^{0.4-}
$$

Furthermore, when the anodic limit of the potential sweep was maintained well below the upper redox charge storage peaks, Burke and Twomey ${ }^{177,211}$ observed a $\mathrm{pH}$ invariant cathodic peak at potentials some $0.3 \mathrm{~V}$ lower than the anodic peak. This peak has also been observed by Lyons and coworkers ${ }^{18,218}$ and is shown clearly in the insets of Fig. 11. The cathodic peak $\mathrm{C} 1$ is reproducibly observed, irregardless of whether the nickel electrode has been subjected to a potential multicycling perturbation or not, provided the upper limit of the potential sweep is reversed at a potential far from the onset of the development of the main charge storage peaks. In relation to this peak, it has been suggested that the reversal in sweep direction at low potentials causes the initially produced hydrated oxide species to transform to a more anhydrous form such as to NiO. ${ }^{177,218}$ In this light the $\mathrm{C} 1$ reduction peak could be due to,

$$
\mathrm{NiO}+\mathrm{H}_{2} \mathrm{O}+2 \mathrm{e}^{-} \rightarrow \mathrm{Ni}+2 \mathrm{OH}^{-}
$$

In essence the proposal put forward by Burke and Twomey ${ }^{177,211}$ is that, due to post electrochemical place exchange reactions, the anionic oxide becomes neutral before being reduced back to Ni metal. In an ellipsometric study de Souza et $a .^{213}$ found that the first layer of oxide formed at potentials close to the lower anodic peak A1 consists of NiO. However this becomes covered with a thick film of $\mathrm{Ni}(\mathrm{OH})_{2}$ upon further increase of potential. Another significant observation of Burke and Twomey $^{177,211}$ was that the magnitude and position of the lower anodic peak did not alter much with repetitive potential cycling. This is supported by the work of Lyons and coworkers ${ }^{18,218}$ presented in Fig. 11. On this basis it has been suggested that it is the inner, compact oxide layer, assuming a duplex layer model, that is reduced at significant cathodic potentials, and thus, even on cycling, the A1 peak is associated with oxidation of $\mathrm{Ni}$ metal to $\mathrm{Ni}(\mathrm{II})$ at the metal/porous hydrous oxide interface.

The complexity of $\mathrm{Ni}(\mathrm{OH})_{2}$ redox chemistry is further highlighted by the presence of two distinct sets of anodic peaks, labelled $\mathrm{A} 2$ and $\mathrm{A} 2{ }^{*}$, and cathodic peaks, labelled $\mathrm{C} 2$ and $\mathrm{C} 2{ }^{*}$, at more elevated anodic potentials. These redox peaks, shown clearly in Fig. 11a for a polycrystalline Ni electrode, are associated with a $\mathrm{Ni}(\mathrm{II}) / \mathrm{Ni}(\mathrm{III})$ transition and represent the main charge storage reaction in Ni hydroxide films. It is noteworthy that a similar voltammetric fine structure has been observed for the electrodeposited $\mathrm{Ni}(\mathrm{OH})_{2}$ material shown previously in Fig. 7. In general, the magnitude of the area under the anodic and cathodic waves increases with repetitive potential cycling corresponding to the formation of a hydrous oxide deposit on the nickel surface. ${ }^{177,212,218}$ It is to be noted that peak A2* disappears with potential multicycling, whereas the cathodic peak doublet $\mathrm{C} 2$ and $\mathrm{C} 2{ }^{*}$ remain even after considerable multicycling, as shown in Fig. 11b. In this way, raising the upper limit of the voltammetric sweep from $-0.20 \mathrm{~V}$ to greater than ca. $0.60 \mathrm{~V}$ ensures the occurrence of further restructuring of the surface layer involving more complete protonation and further uptake of oxygen, resulting not only in increased film thickness but also generation of $\mathrm{Ni}$ (III) or possibly even $\mathrm{Ni}$ (IV) sites in the oxide film. As a consequence of this process, the lower cathodic peak $\mathrm{C} 1$ is no longer observed which suggests the formation of a more stable oxide coating. ${ }^{18}$ The lower limit of the potential sweep has to be extended well into the active hydrogen evolution region before significant reduction of the hydrous oxide occurs. In fact, it has been observed that the optimum lower limit for efficient hydrous oxide formation on $\mathrm{Ni}$ is $c a .-1.45 \mathrm{~V} .^{177}$

A significant point here is that the state of the electrode surface immediately prior to the onset of oxygen evolution is largely dictated by the redox process occurring at the latter anodic peaks. Thus, the specific processes involved in the Ni(II)/ $\mathrm{Ni}(\mathrm{III})$ transition can provide insight into the nature of the OER catalytic sites. A major advance in the understanding of the $\mathrm{Ni}\left(\mathrm{II} / \mathrm{Ni}\right.$ (III) transition was made by Bode et al. ${ }^{173}$ who described the redox behaviour in terms of four phases as illustrated in Fig. 12. The discharged or reduced $\mathrm{Ni}(\mathrm{OH})_{2}$ material can exist either as a largely anhydrous phase designated as $\beta-\mathrm{Ni}(\mathrm{OH})_{2}$ or as a hydrated phase denoted as $\alpha-\mathrm{Ni}(\mathrm{OH})_{2}$. Oxidation of the $\beta-\mathrm{Ni}(\mathrm{OH})_{2}$ material is envisaged to produce a phase referred to as $\beta-\mathrm{NiOOH}$, whereas oxidation of the $\alpha-\mathrm{Ni}(\mathrm{OH})_{2}$ material produces $\gamma$-NiOOH. Hence one expects two distinct redox transitions: $\beta / \beta$ and $\alpha / \gamma$. Here, the corresponding redox peaks are designated $\mathrm{A} 2{ }^{*} / \mathrm{C} 2 *$ and $\mathrm{A} 2 / \mathrm{C} 2$ respectively. It can also be noted from Fig. 12 that upon ageing, especially in more concentrated alkali solution, the $\alpha-\mathrm{Ni}(\mathrm{OH})_{2}$ can dehydrate and re-crystallize as $\beta$-Ni $(\mathrm{OH})_{2}$. Furthermore, upon overcharging at more elevated potentials $\beta$-NiOOH can convert to $\gamma$-NiOOH. The non-stoichiometric nature of both the discharged and charged material is indicated by the average oxidation state of $\mathrm{Ni}$ in each phase as shown in Fig. 12. It is important to note that while 


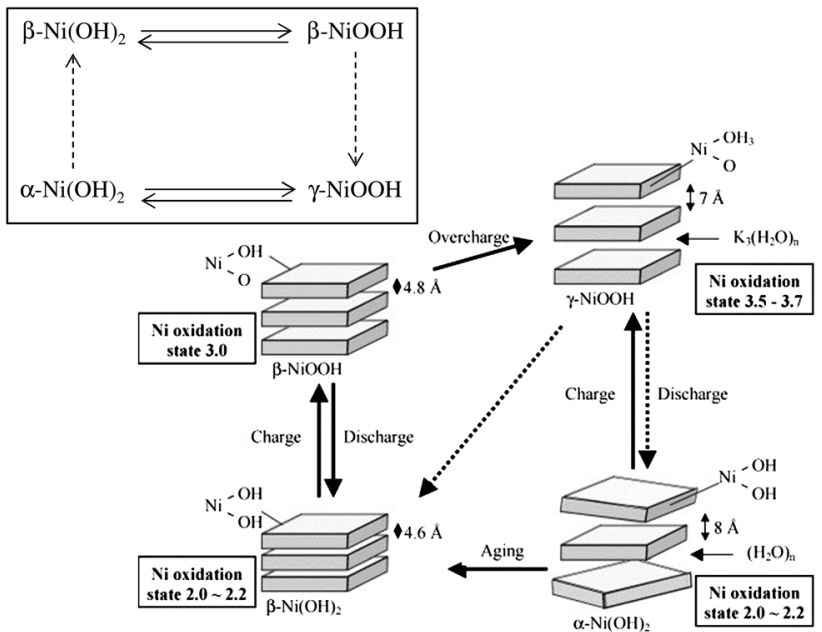

Fig. 12 Schematic representation of the Bode cycle for the $\mathrm{Ni}\left({ }_{1}\right) / \mathrm{Ni}($ III) redox transition in Ni hydroxide layers.

there is a general acceptance for the features of the Bode scheme, it is inappropriate to think about the formation of a compound or a phase with definite stoichiometry during the chemically complex $\mathrm{Ni}(\mathrm{OH})_{2} / \mathrm{NiOOH}$ transformation. Instead the four phases mentioned in the Bode scheme should be considered as the limiting forms of the divalent and trivalent materials, a concept which has recently been reviewed by Lyons and coworkers, ${ }^{46}$ with the actual composition of the oxide at a given potential depending on a range of factors including its history, method of preparation, degree of hydration, defect concentration, etc. An additional point which should also be kept in mind is that the Bode cycle relates specifically to electrodeposited nickel hydroxides and therefore, one must attach a degree of caution in generalising this model to nickel hydroxides prepared by other techniques.

Taking this into consideration, peaks $\mathrm{A} 2^{*} / \mathrm{C} 2^{*}$ can, in simple terms, be attributed to the following $\mathrm{Ni}(\mathrm{II}) / \mathrm{Ni}(\mathrm{III})$ redox transformation,

$$
\mathrm{Ni}(\mathrm{OH})_{2}+\mathrm{OH}^{-} \rightarrow \mathrm{NiOOH}+\mathrm{H}_{2} \mathrm{O}+\mathrm{e}^{-}
$$

Lyons and coworkers ${ }^{167}$ have shown that regular Nernstian potential-pH shifts are typically observed for these peaks hence their association with the anhydrous $\beta / \beta$ transition. On the other hand, super-Nernstian potential $\mathrm{pH}$ shifts of the order of $\mathrm{d} E / \mathrm{dpH}=-2.303(3 R T / 2 F)=-0.088 \mathrm{~V}$ per pH unit at $T=298 \mathrm{~K}$ have been observed for the A2/C2 peaks attributed to an $\alpha / \gamma$ transition, indicating a hydrous or hyper-extended oxide. Therefore, following the reasoning outlined above for $\mathrm{Fe}$, it has been proposed that the main charge storage reaction may be described by,

$$
\begin{aligned}
& {\left[\mathrm{Ni}_{2}(\mathrm{OH})_{6}\left(\mathrm{OH}_{2}\right)_{3}\right]_{n}^{2-}+3 n \mathrm{OH}^{-}} \\
& \quad \rightarrow\left[\mathrm{Ni}_{2} \mathrm{O}_{3}(\mathrm{OH})_{3}\left(\mathrm{OH}_{2}\right)_{3}\right]_{n}{ }^{3-}+3 n \mathrm{H}_{2} \mathrm{O}+2 n \mathrm{e}^{-}
\end{aligned}
$$

corresponding to a $\mathrm{Ni}(\mathrm{II}) / \mathrm{Ni}(\mathrm{III})$ redox transition in a polymeric microdispersed hydrous oxide layer. Interestingly, super-Nernstian shifts of a similar magnitude have also been reported for electrodeposited $\mathrm{Ni}$ hydroxide films. Lyons and coworkers ${ }^{167}$ observed a shift of $c a$. $-0.090 \mathrm{mV}$ per $\mathrm{pH}$ unit for the $\alpha / \gamma$ redox transition in a Ni hydroxide film electrodeposited on a $\mathrm{Au}$ substrate. In light of this, the latter authors postulated that a redox reaction involving anionic, connected surfaquo groups similar to that presented in eqn (43) for the hydrous Ni oxide layer may also operate for the electrodeposited film.

Certainly the redox transition in these films is more complex than that outlined in eqn (42). In a review of EQCM work performed on the $\mathrm{Ni}(\mathrm{OH})_{2} / \mathrm{NiOOH}$ redox transition, WehrensDijksma and Notten ${ }^{219}$ state that during the oxidation of $\mathrm{Ni}(\mathrm{OH})_{2}$ more mass is transferred than can be accounted for by a simple $\mathrm{H}^{+}$extraction as suggested by eqn (42). It is envisaged that this will be accompanied by the incorporation or release of either positive or negative charge compensating ions from the solution and will likely also involve $\mathrm{H}_{2} \mathrm{O}$. A variety of redox models have been proposed to describe the mass changes that take place during the oxidation of $\mathrm{Ni}(\mathrm{OH})_{2}$ and the latter authors have divided these models into two categories: $\mathrm{H}^{+}$transport models or $\mathrm{OH}^{-}$transport models. In the various $\mathrm{H}^{+}$ transport models that have been proposed, the oxidation of $\mathrm{Ni}(\mathrm{OH})_{2}$ takes place in two steps: insertion of cations and deprotonation. Bernard et $a l^{220}$ have proposed a model where, upon oxidation, initially non-hydrated $\mathrm{Ni}(\mathrm{OH})_{2}$ incorporates alkali cations and different amounts of water molecules, depending on the alkali cation present in solution. In the case of initially hydrated $\mathrm{Ni}(\mathrm{OH})_{2}$, Cheek and $\mathrm{O}^{\prime} \mathrm{Grady}^{221}$ have proposed a model where, as oxidation proceeds with cation incorporation, different amounts of water are expelled from the lattice again depending on which alkali cation is present in solution. On the other hand, the application of $\mathrm{OH}^{-}$transport models follow a proposal by Feuilade and Jacoud, ${ }^{222}$ on the basis of radio isotope measurements, that the transport of hydroxide rather than that of protons is predominant in $\mathrm{Ni}(\mathrm{OH})_{2}$ films. In any case, regardless of which model is followed there seems to be general agreement that as oxidation proceeds, alkali metal cations are incorporated into the oxide structure.

\subsection{Interfacial redox chemistry of cobalt based electrodes in alkaline solution}

Cobalt hydroxides were also studied by Bode $e t$ al. ${ }^{173}$ as part of the seminal work that led to the development of the Bode cycle outlined above for the $\mathrm{Ni}$ hydroxide electrodes. In an analogous manner to the situation for divalent Ni hydroxides, there exists both hydrous $\alpha-\mathrm{Co}(\mathrm{OH})_{2}$ and largely anhydrous $\beta-\mathrm{Co}(\mathrm{OH})_{2}$. The structures of these materials are closely related to the corresponding phases in the Ni system. The $\alpha$-phase contains excess $\mathrm{H}_{2} \mathrm{O}$ and has an approximate composition range of $\mathrm{Co}(\mathrm{OH})_{2} \cdot 0.9-1.4 \mathrm{H}_{2} \mathrm{O}$. Benson et al. ${ }^{223}$ found that anodic polarisation of this material produced a phase with an average Co oxidation state of 3.3 which has been recognised as the Co analogue of the $\gamma-\mathrm{NiOOH}$ phase. On the other hand, chemical oxidation in air yielded the $\beta-\mathrm{CoOOH}$ phase. However, as was the case for the Ni hydroxide system discussed previously, the redox behaviour of Co based electrodes is more complicated that this representation suggests. 


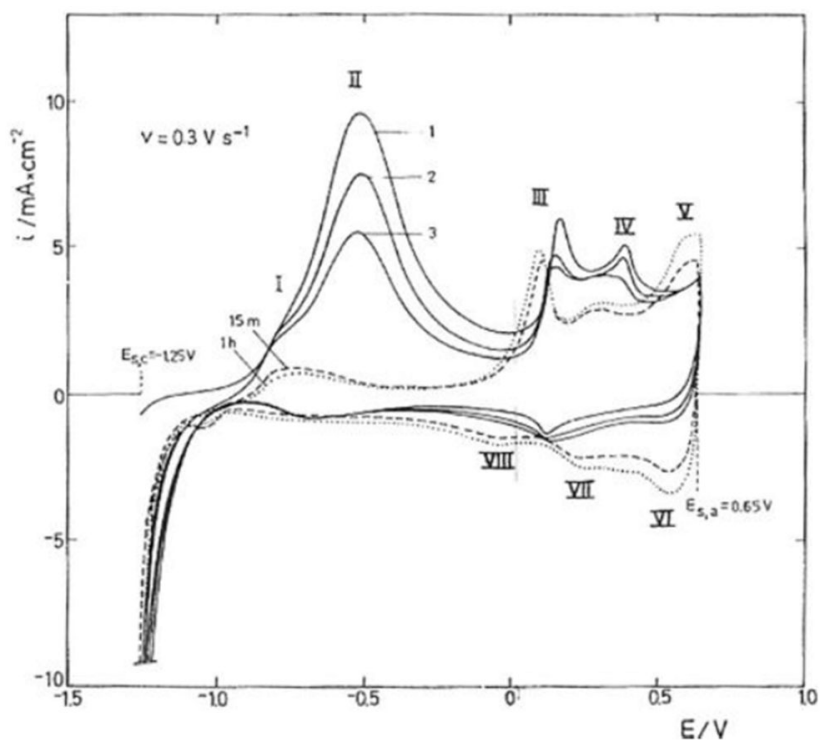

Fig. 13 Cyclic voltammograms recorded in $1.0 \mathrm{M} \mathrm{KOH}$ at $300 \mathrm{mV} \mathrm{s}^{-1}$ for a polycrystalline Co electrode. The first three sweeps and those recorded after $15 \mathrm{~min}$ and $1 \mathrm{~h}$ of cycling are shown. All potentials are referenced to a $\mathrm{Hg} / \mathrm{HgO}$ electrode in $1.0 \mathrm{M} \mathrm{KOH}$

A series of voltammograms recorded for a polycrystalline Co electrode in 1.0 M KOH are presented in Fig. 13. These rather complex voltammetric profiles are typical of those generally observed in the literature, ${ }^{217,224-227}$ and there seems to be fairly good agreement regarding the interpretation of the various peaks. The approach most often adopted is based on that of Behl and Toni $^{224}$ who compared the observed peak potentials with thermodynamic data. In this respect, peak I is attributed to the formation of adsorbed $\mathrm{OH}$ species on the electrode surface,

$$
\mathrm{Co}+\mathrm{OH}^{-} \rightarrow \mathrm{CoOH}_{\mathrm{ads}}+\mathrm{e}^{-}
$$

The initially quite prominent peak II is attributed to a combination of the formation of a passivating film of $\mathrm{Co}(\mathrm{OH})_{2}$ and/or $\mathrm{CoO}$ according to,

$$
\begin{gathered}
\mathrm{Co}+2 \mathrm{OH}^{-} \rightarrow \mathrm{Co}(\mathrm{OH})_{2}+2 \mathrm{e}^{-} \\
\mathrm{Co}+2 \mathrm{OH}^{-} \rightarrow \mathrm{CoO}+\mathrm{H}_{2} \mathrm{O}+2 \mathrm{e}^{-}
\end{gathered}
$$

and a dissolution process forming a slightly soluble $\mathrm{Co}$ (II) species,

$$
\mathrm{Co}+2 \mathrm{OH}^{-} \rightarrow \mathrm{CoO} \cdot \mathrm{OH}_{\mathrm{aq}}{ }^{-}+\mathrm{H}^{+}+2 \mathrm{e}^{-}
$$

This latter assignment is based on the results of a series of rotating ring disk electrode (RRDE) and scanning electrochemical microscopy (SECM) studies performed by Behl and Toni $^{224}$ and Erts et $a .^{228}$ In their RRDE studies, Behl and Toni $^{224}$ observed small but significant amounts of dissolved $\mathrm{Co}^{2+}$ species, the concentration of which reached a maximum at potentials just below those corresponding to peak II in Fig. 13. They noted that the amount of dissolved $\mathrm{Co}^{2+}$ increased with increasing $\mathrm{KOH}$ concentration, whereas no soluble $\mathrm{Co}^{3+}$ or $\mathrm{Co}^{4+}$ species were detected. This data was corroborated by
RRDE work carried out by Erts et $a .^{228}$ They observed that ca. $3 \%$ soluble species were collected in $1.0 \mathrm{M} \mathrm{NaOH}$ in the same potential region as reported by Behl and Toni. ${ }^{224}$ According to Erts et al., ${ }^{228}$ SECM offers a more accurate method of quantification of the concentrations of soluble species, noting that the tip electrode can be located close to the surface and more short lived species can be detected. In $1.0 \mathrm{M} \mathrm{NaOH}$ in the potential region of peak II, they observed that a 'much larger fraction of the reaction proceeds through the dissolution/precipitation path than revealed from RRDE'. The disparity between the SECM results and the RRDE data was explained in terms of a rapid precipitation of the soluble $\mathrm{Co}^{2+}$ species, with the result that much of the soluble species could not escape to the bulk solution and register a ring current. In $0.1 \mathrm{M} \mathrm{NaOH}$ no soluble species were detected by $\mathrm{SECM}^{228}$ indicating a solid-state oxidation mechanism in the less concentrated solution.

In any case, with repetitive potential cycling, it is obvious from Fig. 13 that the magnitude of peak II decreases and the peak potential shifts to more negative values. Interestingly, the magnitude of the corresponding broad cathodic peak at ca. -0.6 to $-0.85 \mathrm{~V}$ is observed to remain approximately invariant. Šimpraga ${ }^{225}$ noted that the ratio of the charges associated with this set of corresponding anodic and cathodic peaks in $0.5 \mathrm{M} \mathrm{NaOH}$ at $298 \mathrm{~K}$ became unity after approximately 15 cycles in the potential range of -1.125 to $-0.325 \mathrm{~V}$. Similar observations have also been made by other workers. ${ }^{217,226,227}$ This behavior has been attributed to the stabilization of the developing anodic film with respect to the dissolution process of eqn (47), so that after a number of cycles the anodic peak in the potential region of -0.6 to $-0.8 \mathrm{~V}$ should be almost entirely due to a solid state process.

The nature of the passive layer formed on Co in alkaline solution has been studied using ellipsometry. ${ }^{229,230}$ The most general conclusion of these studies was that cobalt forms two types of passive layers. The first is formed at lower potentials, has a $\mathrm{pH}$ dependent thickness, and consists of $\mathrm{Co}(\mathrm{OH})_{2}$ and $\mathrm{CoO}$. A second passive layer is formed outside the Co(II) layer at potentials above $c a .0 \mathrm{~V}(v s . \mathrm{Hg} / \mathrm{HgO})$ and is envisaged to consist of $\mathrm{Co}(\mathrm{III})$ and possibly $\mathrm{Co}(\mathrm{IV})$ species. XPS measurements by Foelske et al. ${ }^{231}$ on polycrystalline Co in $0.1 \mathrm{M} \mathrm{NaOH}$ further emphasize the concept of a twin layer type passive film. Below the potential region corresponding to peak III in Fig. 13 the passive layer is described as being a $\mathrm{Co}(\mathrm{OH})_{2}$ film, which can grow to $40 \mathrm{~nm}$ thickness, depending on time and potential. Significantly, the authors claim that no CoO was detected, at least in the early stages of passivation. At potentials anodic to peak III the outer layers of the film are said to be dominated by $\mathrm{Co}$ (III) species, with the XPS measurements identifying $\mathrm{Co}_{3} \mathrm{O}_{4}$ and $\mathrm{CoOOH}$. Furthermore, in an XPS investigation of a thick hydrous oxy-hydroxide layer formed by multi-cycling a polycrystalline Co electrode in $0.5 \mathrm{M} \mathrm{NaOH}$ over a potential range of $650 \mathrm{mV}$ up to just below significant oxygen evolution, the dominant species identified was $\mathrm{Co}_{3} \mathrm{O}_{4}{ }^{225}$

In view of this, Peaks III and IV are attributed to the oxidation of Co(II) species to Co(III) species. It is obvious that the voltammetric profile in this region changes significantly 
with cycling tending eventually to a reversible type behaviour. In particular, Liu et al. ${ }^{226}$ have shown that the formation and reduction of the oxide film on $\mathrm{Co}$ in $0.5 \mathrm{M} \mathrm{NaOH}$ progresses from a completely irreversible behaviour during early potential cycles to a largely reversible behaviour upon the formation of a thick hydrous oxide with extensive cycling. Two "mirror image" reversible redox peaks, with peak potentials of $c a .525 \mathrm{mV}$ and $225 \mathrm{mV}$ (vs. $\mathrm{Hg} / \mathrm{HgO}$ ) were observed to become increasingly well resolved from about the 600th voltammetric cycle at $20 \mathrm{mV} \mathrm{s}^{-1}$ through to the 2885th cycle. It was found that the amount of anodic charge reversibly accessible in the 2885th cycle was only $3 \%$ of the total accumulated charge over the course of the film growth. This led the authors to conclude that, for this thick hydrous oxide film, it is only a surface region layer that displays accessible redox activity.

On the first voltammetric sweep, thermodynamic data indicates that the oxidation of $\mathrm{Co}$ (II) species should begin in the region of $-0.4 \mathrm{~V}$ (vs. $\mathrm{Hg} / \mathrm{HgO})$ with $\mathrm{Co}_{3} \mathrm{O}_{4}$ being the initial oxidation product. Gomez Meier et al. ${ }^{227}$ envisage this process in terms of the oxidation of an inner $\mathrm{CoO}$ layer to $\mathrm{Co}_{3} \mathrm{O}_{4}$ according to,

$$
3 \mathrm{CoO}_{\text {inner }}+\mathrm{H}_{2} \mathrm{O} \rightarrow \mathrm{Co}_{3} \mathrm{O}_{4}+2 \mathrm{H}^{+}+2 \mathrm{e}^{-}
$$

while Burke et $a .^{217}$ commented that an illustrative rationalisation of the process could be provided by the reaction,

$$
3 \mathrm{Co}(\mathrm{OH})_{2}+2 \mathrm{OH}^{-} \rightarrow \mathrm{Co}_{3} \mathrm{O}_{4}+4 \mathrm{H}_{2} \mathrm{O}+2 \mathrm{e}^{-}
$$

Relating this to peaks III and IV in Fig. 13 Burke et al. ${ }^{217}$ suggested, on the basis of the large background currents in the anodic sweep in the region above about $-0.3 \mathrm{~V}$, that a hydrous $\mathrm{Co(II)} \mathrm{film} \mathrm{was} \mathrm{gradually} \mathrm{converted} \mathrm{to} \mathrm{such} \mathrm{Co}$ (III) containing species as $\mathrm{Co}_{3} \mathrm{O}_{4}, \mathrm{Co}_{2} \mathrm{O}_{3}$ and $\mathrm{CoOOH}$. They point out that, the situation is further complicated by a number of other factors, such as the existence of three forms of $\mathrm{CoOOH}$; $\mathrm{CoOOH}(\mathrm{I})$, $\mathrm{CoOOH}(\mathrm{II})$ and $\mathrm{CoHO}_{2}$. These authors add that hydration effects will tend to render inaccurate the assignment, based on thermodynamic data, of closely spaced peaks to specific $\mathrm{Co}(\mathrm{II}) / \mathrm{Co}(\mathrm{III})$ transitions.

Gomez Meier et al. $^{227}$ also allude to the complex hydrous nature of the anodic oxide formed on Co in alkaline solution. These authors view the oxide growth process in terms of the formation of 'sandwich-type' structures, with a $\mathrm{Co} / \mathrm{CoO} /$ $\mathrm{Co}(\mathrm{OH})_{2}$ 'sandwich' formed at lower potentials. They envisage peak III to represent the oxidation of a hydrous form of this structure to a new hydrated 'sandwich' structure according to the process,

$$
\begin{aligned}
{\left[\mathrm{Co}(\mathrm{CoO})\left(\mathrm{Co}(\mathrm{OH})_{2}\right) \cdot x \mathrm{H}_{2} \mathrm{O}\right] \rightarrow } & {\left[\mathrm{Co}(\mathrm{CoO})(\mathrm{CoOOH}) \cdot x^{\prime} \mathrm{H}_{2} \mathrm{O}\right] } \\
& +\mathrm{H}^{+}+x^{\prime \prime} \mathrm{H}_{2} \mathrm{O}+\mathrm{e}^{-}
\end{aligned}
$$

where $x^{\prime \prime}=x^{\prime}+x$. The nature of this 'sandwich' structure will further change with increasing potential, the $\mathrm{CoO}$ component being oxidised to $\mathrm{Co}_{3} \mathrm{O}_{4}$. According to this viewpoint, peak IV will then correspond to a process along the lines of,

$$
\left[\mathrm{Co}\left(\mathrm{Co}_{3} \mathrm{O}_{4} \cdot \mathrm{CoOOH}\right)\right]+2 \mathrm{H}_{2} \mathrm{O} \rightarrow\left[\mathrm{Co}(\mathrm{CoOOH})_{4}\right]+\mathrm{H}^{+}+\mathrm{e}^{-}
$$

Furthermore, on the basis of a perceived correlation between their voltammetric peak data and the predictions of thermodynamics, Gomez Meier et $a .^{227}$ conclude that, in the formation of the anodic film on Co, the electrochemical reactions involve fast proton transfers and that the advance in the degree of oxidation of the metal is accompanied by a deprotonation and change in the water content at different parts of the complex electrochemical interface.

It is generally agreed that Peak V is due to a surface $\mathrm{Co}$ (III) to Co(Iv) oxidation. Burke et al. ${ }^{217}$ attribute an electrochromic effect observed in this potential region to a $\mathrm{Co}(\mathrm{III})_{\text {orange/ }}$ $\mathrm{Co}(\mathrm{Iv})_{\text {brown }}$ transition according to,

$$
\mathrm{CoOOH}+\mathrm{OH} \rightarrow \mathrm{CoO}_{2}+\mathrm{H}_{2} \mathrm{O}+\mathrm{e}^{-}
$$

The latter authors note that the charge associated with peak $\mathrm{V}$ increases on cycling. In fact, the cathodic peak VI corresponding to the reduction of Co(Iv) species is virtually absent on the first cycle; peaks V and VI only become well defined at appreciable film thickness. This observation in tandem with the conclusions of Liu et al., ${ }^{226}$ that the accessible reversible charge in a thick Co oxyhydroxide film resides adjacent to the surface, indicates very strongly that the oxidation of $\mathrm{Co}$ (III) to $\mathrm{Co}$ (Iv) species is predominantly a surface process, occurring at the interface between a hydrous oxide layer and the solution phase. In the context of the present work, this formation of surface Co(rv) species is likely to be quite significant, occurring as it does at potentials immediately below those associated with a significant oxygen evolution current.

The relatively small magnitude of the charge associated with the cathodic peaks in Fig. 13 during the early potential cycles indicates that the initially formed passivating film is not easily reduced. Peak VIII and the broad ill-defined cathodic peak at potentials between approximately -0.4 and $-0.8 \mathrm{~V}$ are generally attributed to the reduction of $\mathrm{Co}$ (III) species. ${ }^{217,226,227,232}$ Although it is not particularly apparent in Fig. 13, probably owing to the relatively fast scan rate at which these CVs were recorded, most workers have observed a reasonably well-defined peak at $c a$. $-1.0 \mathrm{~V}$ associated with the reduction of $\mathrm{Co}$ (II) species. $^{217,226,227,232}$ As previously described, Liu et al. ${ }^{226}$ have attributed the increasing reversibility achieved with increasing film thickness to redox processes in the vicinity of the hydrous oxide surface. The contrast between this behaviour and the irreversibility noted during the earlier stages of film formation reinforces the concept of the initially formed oxide or inner regions of a thick film being difficult to reduce.

\section{Charge transport in transition metal oxides}

The charge transport properties of transition metal oxides are of fundamental importance for the potential applications of these systems. Metal oxide electrode materials have been implicated in a range of energy production and energy storage devices, from the electrolytic splitting of water ${ }^{13}$ to various fuel cells, $^{233}$ batteries $^{209}$ and supercapacitors. ${ }^{234,235}$ Although the 


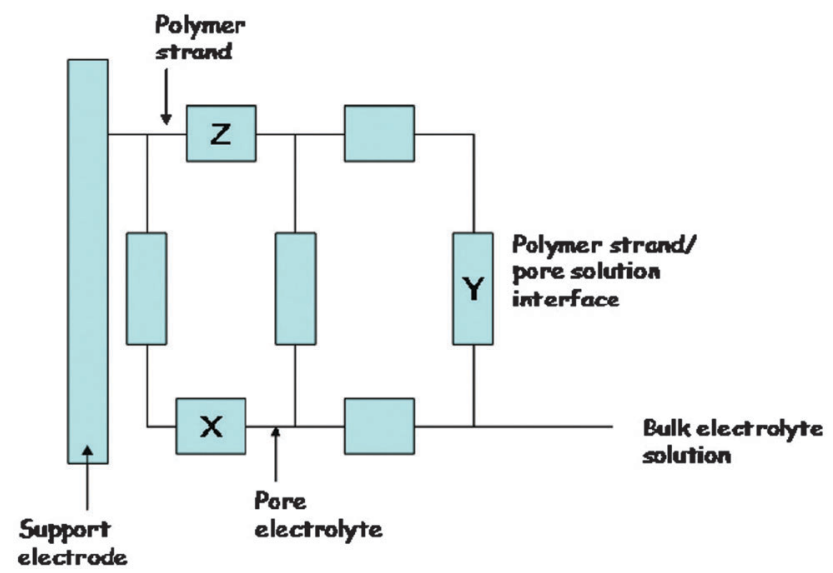

Fig. 14 Dual rail transmission line model for mixed ionic/electronic conduction in metal oxide materials.

specifics of each technology may differ, three key properties can be identified for an optimal electrode material: a high electrochemically active surface area, fast electron transfer kinetics, and facile and reversible ion diffusion. For metal oxide based electrodes the latter properties are intrinsically linked to the redox switching behaviour of the film. Therefore, an understanding of the kinetics and mechanism of the redox switching reactions is essential for the development and design more effective electrocatalytic and charge storage materials.

Redox switching in metal oxide based materials reflects the potential induced change in oxidation state of the film and involves the transport of both electrons and counterions. In this way, the metal oxides may be viewed as mixed ionic/electronic conductors similar to electroactive redox and conducting polymers. The physical transport processes within the material may be described in terms of a dual rail electrical transmission line, ${ }^{236-240}$ a general schematic of which is presented in Fig. 14. This model consists of a series of distributed impedances corresponding to charge transport along the polymer strand $\mathrm{Z}$, ion transport within the pore electrolyte $\mathrm{X}$, and an impedance $\mathrm{Y}$ representing the interface between the strand and the pore. At the simplest level $\mathrm{Z}$ corresponds to the electronic resistance $R_{\mathrm{E}}, \mathrm{X}$ to the ionic resistance $R_{\mathrm{I}}$, and $\mathrm{Y}$ to a distributed capacitance $C_{\Sigma}$. The application of this type of circuit has been discussed in detail by Bisquert and coworkers ${ }^{236-238}$ in relation to electronically conducting polymers and transition metal oxide materials.

Charge transport information may also be obtained from the voltammetric characteristics of a system. On a qualitative level, the voltammetric charge arising from the surface redox transitions gives an indication of the relative kinetics of the redox reaction and the extent to which it percolates throughout the film. It has been experimentally established that the measured value of the voltammetric charge depends on the potential sweep rate with $Q$ or $Q^{*}$ increasing significantly in magnitude as the sweep rate decreases. ${ }^{181}$ This trend can be observed clearly in Fig. 15 where the value of $Q$ determined for a series of hydrous $\mathrm{Fe}$ oxide films is plotted as a function of sweep rate. These results may be rationalized in terms of a simple

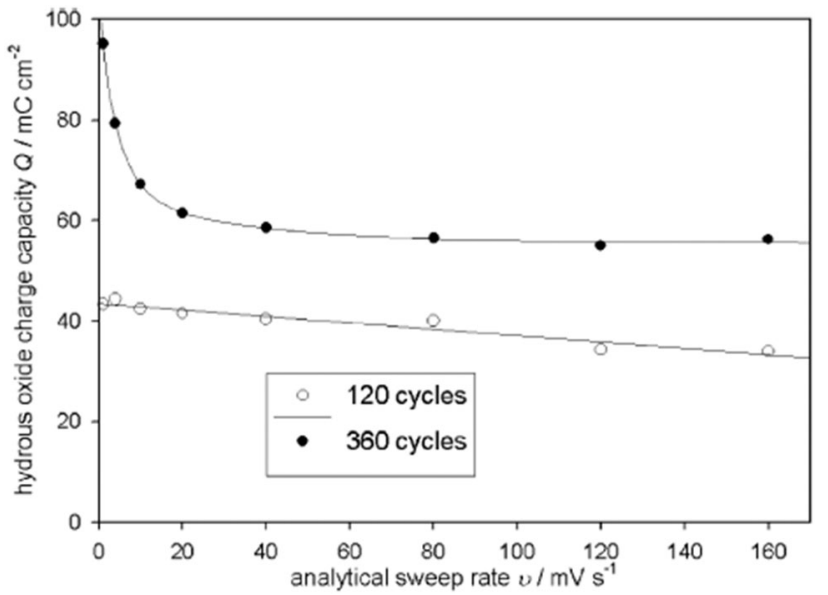

Fig. 15 Variation of oxide charge capacity $Q$ with analytical sweep rate for hydrous Fe oxide coated electrodes in $1.0 \mathrm{M} \mathrm{NaOH}$. The hydrous oxide layers were prepared using 120 and 360 growth cycles in $1.0 \mathrm{M} \mathrm{NaOH}$ at $350 \mathrm{mV} \mathrm{s}^{-1}$ between the switching potentials of -1.45 and $0.35 \mathrm{~V}$ vs. $\mathrm{Hg} / \mathrm{HgO}$.

diffusional model. In relation to the electrocatalytic and redox properties exhibited by thermally prepared $\mathrm{RuO}_{2}$ and $\mathrm{IrO}_{2}$ electrodes in aqueous acid and base solutions, Trasatti and co-workers ${ }^{181}$ have differentiated between an 'inner' or less accessible active surface and an outer or more accessible active surface. In their deliberations the charge capacity derived from the voltammetric profile was presumed to contain contributions which reflected the differing degrees of access of protons to the outer and inner regions of the oxide lattice. In this sense, these results suggest that increasing fractions of the inner surface of the oxide film become electroactive as the experimental timescale increases. As noted by Lyons and Floquet, ${ }^{75}$ this likely occurs due to the diffusion of water molecules through cracks, pores and grain boundaries with subsequent acid/base reactions between bound metal oxyhydroxide groups and protons according to,

$$
\mathrm{MO}_{x}(\mathrm{OH})_{y}+\delta \mathrm{H}^{+}+\delta \mathrm{e}^{-} \rightarrow \mathrm{MO}_{x-\delta}(\mathrm{OH})_{y+\delta}
$$

Indeed, Trasatti and co-workers ${ }^{241}$ have described the proton diffusion process in terms of a surface Grotthus mechanism along the pore and crack walls, with this process being facilitated by the presence of readily dissociatable surface $\mathrm{OH}$ groups. Hence, by analogy with the situation pertaining in solution, it is to be expected that the mobility of $\mathrm{H}^{+}$is considerably higher than that of $\mathrm{OH}^{-}$and so access to inner surface sites is easier for free protons than for other proton donating species. Actually, Lyons and Floquet ${ }^{75}$ have recently observed for a series of $\mathrm{RuO}_{2}$ and $\mathrm{IrO}_{2}$ based electrodes that larger $Q^{*}$ values are obtained in acidic solutions than in alkaline solutions, indicating a more efficient charge transport process in acidic media.

In the hydrous and bulk oxide layers the diffusional process may be described in terms of an electron 'hopping' mechanism. ${ }^{17}$ Redox centres immediately adjacent to the support electrode are directly affected by the electrode potential, 
whereas charge is further propagated along the oxy-metal strands via a sequence of electron self exchange reactions between neighbouring oxy-metal sites. So, for the thinner films represented in Fig. 15 there is sufficient time at normal sweep rates for the redox reaction to extend to virtually all regions of the dispersed hydrous layer. That is, a situation of Nernstian equilibrium prevails and the voltammetric charge is relatively unaffected by the sweep rate. However, for the thicker films there is not enough time, apart from at very low scan rates, for the redox reaction to propagate throughout the entire extent of the dispersed hydrated region. Thus, the charge capacity values drop dramatically as the analytical sweep rate is increased. Similar quasi-diffusive effects have been discussed for a number of electroactive polymer systems. ${ }^{205,242-246}$

In light of the above discussion, the rate of charge percolation in the oxide layers may be quantified in terms of a charge transport diffusion coefficient $D_{\mathrm{CT}}$, reflecting either the rate of electron 'hopping' or the diffusion of protons/hydroxide ions via a rapid Grotthus type mechanism. ${ }^{17}$ In this sense, Laviron ${ }^{247-252}$ and Aoki ${ }^{253,254}$ have developed useful models allowing the voltammetric response to be analysed quantitatively as a function of sweep rate. In particular, Laviron and coworkers ${ }^{24-252}$ derived general expressions for the cathodic or anodic voltammetric peaks for any degree of reversibility,

$$
\begin{aligned}
& \Psi_{\text {cath }}=m \eta^{-\alpha}(1+\eta) \exp [f(\eta)] \int_{\infty}^{\eta} u^{-(1+\alpha)} \exp [-f(u)] \mathrm{d} u \\
& \Psi_{\text {an }}=-m \eta^{-\alpha}[\eta-m(1-\eta)] \exp [f(\eta)] \int_{\infty}^{\eta} u^{-\alpha} \exp [-f(u)] \mathrm{d} u
\end{aligned}
$$

Here, $\Psi$ denotes an adimensional current given by,

$$
\Psi=\frac{i}{\left(\frac{F^{2}}{R T}\right) n^{2} v A \Gamma_{\mathrm{T}}}
$$

where $\Gamma_{\mathrm{T}}$ denotes the total surface concentration of reactant and product and $m$ is related to the sweep rate $v$ according to,

$$
m=\frac{R T}{F\left(\frac{k}{n v}\right)}
$$

where $k$ denotes the rate constant for the surface process. In the latter expressions, the quantity $\eta$ is related to the potential according to,

$$
\eta=\exp \left[n F\left(E-E_{\Sigma}^{0}\right) / R T\right]
$$

and $f(\eta)$ is given by,

$$
f(\eta)=[m / \alpha(1-\alpha)] \eta^{-\alpha}[1-\alpha(1+\eta)]
$$

In addition we note that $u$ denotes a dummy integration variable and the other symbols have their usual meaning.

Specifically, Lyons and Burke ${ }^{57}$ have shown that for the $\mathrm{Ru}(\mathrm{VI}) / \mathrm{Ru}(\mathrm{VII})$ redox transition in a thermally prepared $\mathrm{RuO}_{2}$ layer the general expressions outlined in eqn (54) and (55) reduce to the simple reversible form,

$$
\Psi=\frac{i}{\left(\frac{n^{2} F^{2}}{R T}\right) A \Gamma_{\mathrm{T} v}}=\frac{\eta}{(1+\eta)^{2}}
$$

for the limiting situation as $m \rightarrow \infty$. It may be shown that this function exhibits a maximum for $\eta=1$. Hence, examination of eqn (58) implies that the peak potential $E_{\mathrm{p}}=E_{\Sigma}^{0}$, where $E_{\Sigma}^{0}$ is the standard potential for the surface redox process. Now, setting $\eta=1$ in eqn (60) yields the following expression for the peak current $i_{\mathrm{p}}$,

$$
i_{\mathrm{p}}=\frac{n^{2} F^{2} A}{4 R T} \Gamma_{\mathrm{T}} v
$$

Thus, the peak current for the surface redox process is expected to vary linearly with the sweep rate; a prediction that has been verified experimentally for a number of thermally prepared $\mathrm{RuO}_{2}$ and $\mathrm{IrO}_{2}$ based oxide layers. ${ }^{190-193}$

This analysis has been further developed by Aoki and co-workers ${ }^{253,254}$ who have shown that the peak current varies with sweep rate according to,

$$
i_{\mathrm{p}}=0.446 n F A\left\{\frac{\Gamma_{\Sigma} D_{\mathrm{CT}}}{L^{2}}\right\} W^{1 / 2} \tanh Y
$$

where,

$$
W=\frac{n F L^{2} v}{D_{\mathrm{CT}} R T}
$$

$$
Y=0.56 W^{1 / 2}+0.05 W
$$

and $n, L, A$ and $\Gamma_{\Sigma}$ represent the number of electrons transferred in the redox process, the thickness of the oxide layer, the geometric area of the electrode and the surface coverage of the active oxy-metal groups in the surface layer respectively. The latter quantity is related to the voltammetric charge $Q$ and the redox site concentration $c_{\Sigma} v i a$,

$$
\Gamma_{\Sigma}=c_{\Sigma} L=Q / n F A
$$

Two limiting forms of the general expression outlined in eqn (62) may be identified. For low values of sweep rate corresponding to small $W$, we can set $\tanh Y \approx Y$ in eqn (62) to obtain,

$$
i_{\mathrm{p}}=\frac{n^{2} F^{2} A}{4 R T} \Gamma_{\Sigma} v
$$

which is equivalent to the Laviron analysis in eqn (61). On the other hand, for fast sweep rates corresponding to large $W$ values we set $\tanh Y \approx 1$ and obtain,

$$
i_{\mathrm{p}}=0.446\left\{\frac{(n F)^{3}}{R T}\right\}^{1 / 2} A D_{\mathrm{CT}}^{1 / 2} c_{\Sigma} v^{1 / 2}
$$

The important point to note here is that diffusion coefficients may only be evaluated at high sweep rates, where concentration 
polarization effects due to incomplete titration of redox sites are manifested.

Lyons and coworkers ${ }^{17,92}$ have applied the Aoki model to the voltammetric data obtained for hydrous Fe oxide coated electrodes. In particular, they note that the $i_{\mathrm{p}} / v^{1 / 2}$ proportionality can be readily obtained at reasonable values of sweep rate for thick layers whereas the $i_{\mathrm{p}} / v$ proportionality, suggesting the operation of Nernstian equilibrium throughout the dispersed layer during redox switching, is observed over an extended range of sweep rates for thin layers. These general conclusions are illustrated in Fig. 16 and 17 where the redox peak current data recorded for hydrous Fe oxide layers prepared using 30 and 240 growth cycles respectively are presented. It can be seen from the thin film data in Fig. 16a that excellent linearity is observed between the peak current and scan rate for the hydrous oxide peaks A3 and $\mathrm{C} 2$, and the compact oxide reduction peak $\mathrm{C} 1$ over an extended scan rate window. This observation is confirmed in the double logarithmic analysis of the same data in Fig. 16b, where slopes of greater than 0.8 are noted for the $\mathrm{A} 3$ and $\mathrm{C} 2$ peaks. In contrast, the data obtained for the thicker layers varies linearly with $v^{1 / 2}$ except at relatively low sweep rates, as shown in Fig. 17a. In this case, the double
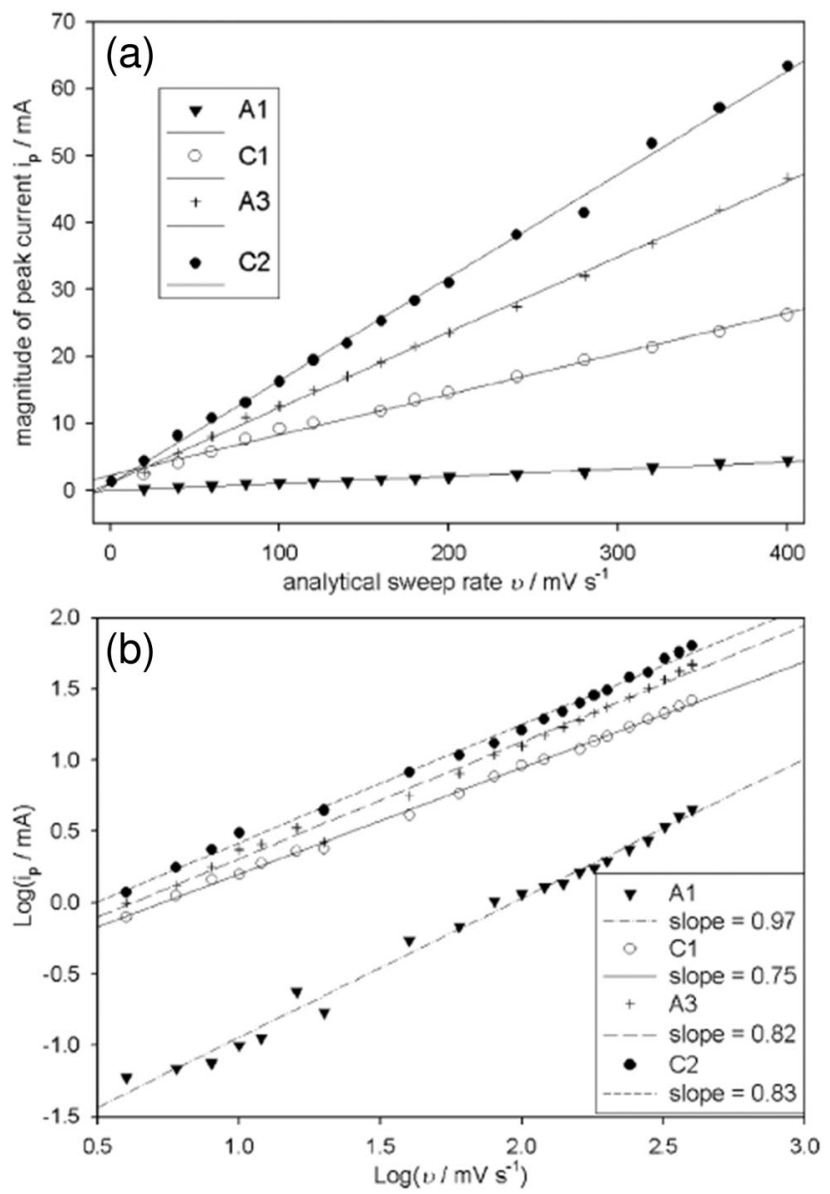

Fig. 16 (a) Variation of the voltammetric peak current with sweep rate for a hydrous Fe oxide layer prepared as in Fig. 15 using 30 growth cycles. (b) $\log i_{p} /$ $\log v$ plot of the data outlined in (a) above.
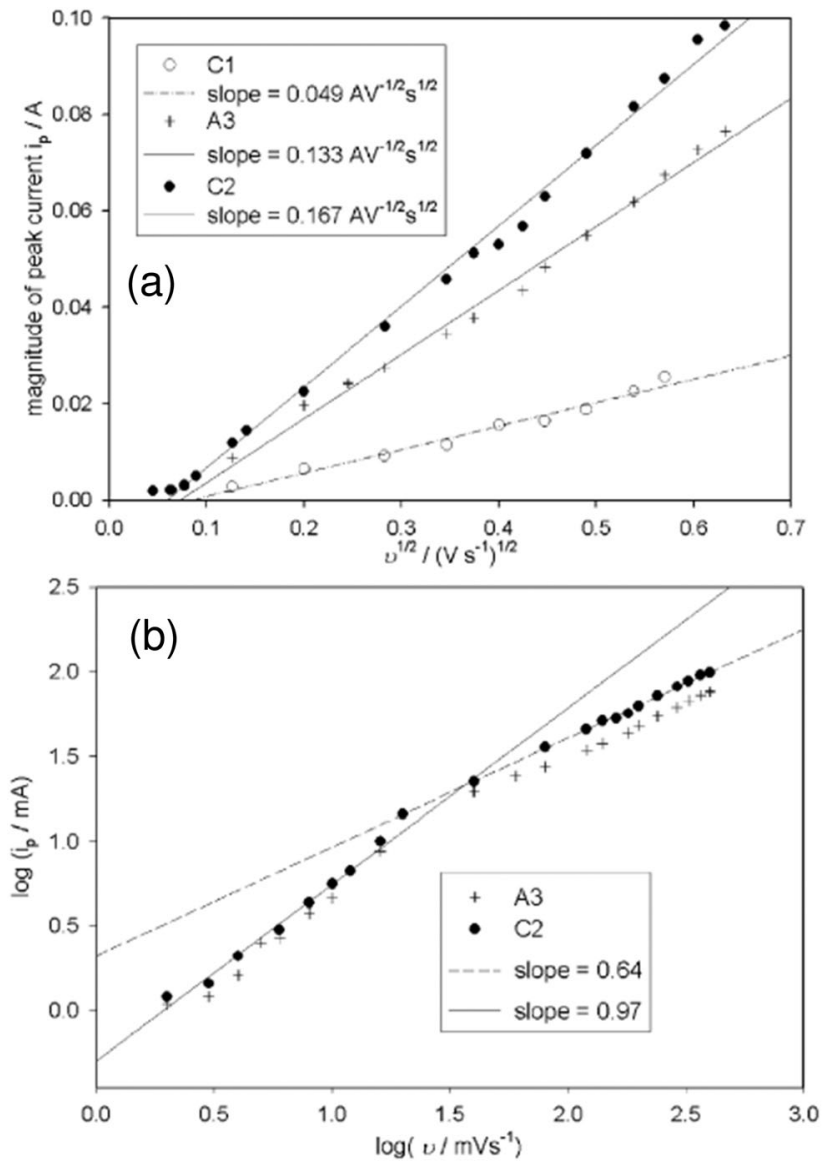

Fig. 17 Variation of the voltammetric peak potential with $v^{1 / 2}$ for a hydrous Fe oxide layer prepared as before using 240 growth cycles. (b) $\log i_{p} / \log v$ plot of the data outlined in (a) above.

logarithmic analysis shown in Fig. 17b is particularly interesting in that a dual slope behaviour is observed; at low sweep rates a slope of 0.97 is obtained whereas at higher sweep rates the slope is 0.64 . Thus, the sweep rate at which concentration polarization effects become important may be readily discerned from the break point in this "dog's leg" curve and transport information in the form of a $D_{\mathrm{CT}}$ value may be extracted from the data in the region above this point. Based on the slopes for the $\mathrm{A} 3$ and $\mathrm{C} 2$ peaks in the Randles-Sevcik plots in Fig. 17a, Lyons and coworkers ${ }^{17,97}$ calculated a $D_{\mathrm{CT}}$ value of ca. $2.8 \pm 0.9 \times 10^{-10} \mathrm{~cm}^{2} \mathrm{~s}^{-1}$ for the hydrous Fe oxide layer.

In the latter calculation, a number of assumptions were admitted and so, the value reported for $D_{\mathrm{CT}}$ can only be considered an approximation. The concentration of redox sites in the oxide layer was effectively given by,

$$
c_{\Sigma}=\rho / M
$$

where $\rho$ denotes the density of the hydrous material and $M$ represents the molar mass of the fundamental repeat unit of the hydrated oxy-iron polymer. Here, the densities of the oxidized and reduced forms of the hydrous layer were assumed to be given by known values for $\mathrm{Fe}(\mathrm{OH})_{2}$ and $\mathrm{Fe}_{2} \mathrm{O}_{3} \cdot n \mathrm{H}_{2} \mathrm{O}$ respectively. ${ }^{180,205,255}$ Furthermore, the molar masses for the 
oxidized and reduced states were calculated from the surfaquo group species presented in eqn (35). Although this representation is reasonable, it is important to remember that these species were proposed in order to rationalize the observed super-Nernstian potential-pH response and should not be considered absolute. However, it is important note that the $D_{\mathrm{CT}}$ value reported by Lyons and coworkers is not unreasonable and compares well with those reported for electroactive polymers such as polyaniline $\left(3 \times 10^{-9} \mathrm{~cm}^{2} \mathrm{~s}^{-1}\right)^{247-252}$ and polypyrrole $\left(1 \times 10^{-8} \mathrm{~cm}^{2} \mathrm{~s}^{-1}\right),{ }^{256-261}$ and other hydrated oxides such as hydrous iridium oxide $\left(1.5 \times 10^{-9} \mathrm{~cm}^{2} \mathrm{~s}^{-1}\right.$, acidic solution). ${ }^{176,262}$ Therefore, we suggest that cyclic voltammetry offers a facile and yet versatile approach to the study of charge transport kinetics in metal oxide based materials.

\section{Electrocatalytic properties of transition metal oxides: the oxygen evolution reaction}

In our discussion of the electrocatalytic properties of transition metal oxide electrodes we will focus on the technologically important oxygen evolution reaction (OER). The generation of molecular oxygen via water electrolysis is a complex and energetically demanding reaction. In alkaline solution, the overall halfcell reaction for the OER may be represented as,

$$
4 \mathrm{OH}^{-} \rightarrow \mathrm{O}_{2}+2 \mathrm{H}_{2} \mathrm{O}+4 \mathrm{e}^{-}
$$

with a reversible potential in alkaline solution of $0.303 \mathrm{~V} v s . \mathrm{Hg}$ / HgO. ${ }^{13,233}$ However, in order to generate oxygen at an appreciable rate an overpotential of several hundred millivolts may be required. Indeed it has been shown that, at a given operational current density, this relatively large anodic oxygen evolution overpotential is the principal factor limiting the efficiency of industrially important cathodic processes such as metal electrowinning and hydrogen production via alkaline water electrolysis. 4,6,7,9,263,264 Consequently, there has been considerable research effort devoted to decreasing oxygen evolution overpotentials, through the development of novel anode materials such as those outlined above, and to the elucidation of possible mechanistic pathways at these electrode surfaces. ${ }^{10,11,46}$

In this context, we have adopted a classical electrochemical approach in our examination of the kinetics of the OER at metal oxide and oxidized metal electrodes. This approach typically involves the use of an ensemble of steady state and transient electrochemical techniques such as Steady-State Polarisation curves, Open Circuit Potential Decay (OCPD) and Electrochemical Impedance Spectroscopy (EIS). Our aim here is not to play down the importance of modern non-electrochemical spectroscopic $^{265-269}$ and computational methods, ${ }^{270-273}$ rather we wish to highlight the potential usefulness of a relatively simple and unsophisticated electrochemical approach. Such electrochemical methods have been shown to be extremely effective analytical tools, yielding key parameters such as the Tafel slope $b$ and the reaction order $m$ of the mechanistically significant reactant. In the present discussion we focus on the important practical considerations relating to the application of these techniques in a kinetic study of the OER. Building on this, we examine how the latter kinetic parameters can be used to propose a chemically meaningful mechanism for the multistep OER. Finally, the evaluation of turnover frequencies is outlined as a useful tool in the electrocatalytic comparison of metal oxide based OER anodes.

\subsection{Kinetic studies of the OER}

(a) Steady-state polarisation. Steady-state Tafel plot analysis is the most widely applied electrochemical technique in the electrocatalytic study of reactions such as the OER. In general terms the Tafel equation may be written as,

$$
\eta=a+b \log i
$$

implying that a plot of overpotential $\eta$ as a function of the current $i$ in logarithm form will be linear with a Tafel slope $b$ and intercept $a \cdot{ }^{274}$ Under Nernstian conditions, the exchange current density $i_{0}$ and thus the standard heterogeneous rate constant $k^{0}$ may theoretically be calculated from the intercept $a$. However, owing to the extreme irreversibility of the oxygen evolution process, it has long been appreciated that the principle of microscopic reversibility is unlikely to apply to the oxygen electrode. ${ }^{275}$ As a consequence, steady state kinetic data on the OER can only yield mechanistic information up to and including the rate-determining step (RDS).

In the case of multistep reactions such as the OER the experimentally observed Tafel slope can be shown to be, ${ }^{276-280}$

$$
b=2.303\left(\frac{R T}{\vec{\alpha} F}\right)
$$

where $R, T$ and $F$ have their usual meanings and the transfer coefficient $\vec{\alpha}$ is given by,

$$
\vec{\alpha}=\frac{\vec{n}}{\nu}+n_{\mathrm{r}} \beta
$$

where $\vec{n}$ is the number of electrons transferred before the RDS, $\nu$ is the stoichiometric number defined as the number of times the RDS occurs for one repetition of the overall reaction, $n_{\mathrm{r}}$ is the number of electrons transferred in each occurrence of the RDS and $\beta$ is the symmetry factor, generally taken to be $1 / 2$, which arises from the activation of vibrational states and the energy required to break bonds. In this way, it can be seen that the Tafel slope is a composite parameter, giving information on the stoichiometry and the succession of steps in the overall reaction. For example, if the first electron transfer step in a sequential reaction is rate-determining then $n_{\mathrm{r}}=1, \vec{n}=0$ and $\vec{\alpha}=$ $\beta=1 / 2$ implying a Tafel slope of $c a .120 \mathrm{mV} \mathrm{dec}^{-1}$ at $25{ }^{\circ} \mathrm{C}$. Similarly, if the second electron transfer step is rate-determining $n_{\mathrm{r}}=1$ and $\vec{n}=1$ and, assuming $\nu=1$, we get $\vec{\alpha}=3 / 2$ giving a Tafel slope of $c a .40 \mathrm{mV} \mathrm{dec}{ }^{-1}$ at $25{ }^{\circ} \mathrm{C}$. On the other hand, if the ratedetermining step involves a chemical step subsequent to the first electron transfer step we can set $n_{\mathrm{r}}=0$ and $\vec{n}=1$. In this case, the $\beta$ term is removed and, again assuming that $\nu=1$, we can show that $\vec{\alpha}=1$ predicting a Tafel slope of $c a .60 \mathrm{mV} \mathrm{dec}{ }^{-1}$ at $25{ }^{\circ} \mathrm{C}$. Thus, the elucidation of Tafel slopes can be useful in differentiating between possible reaction mechanisms. 


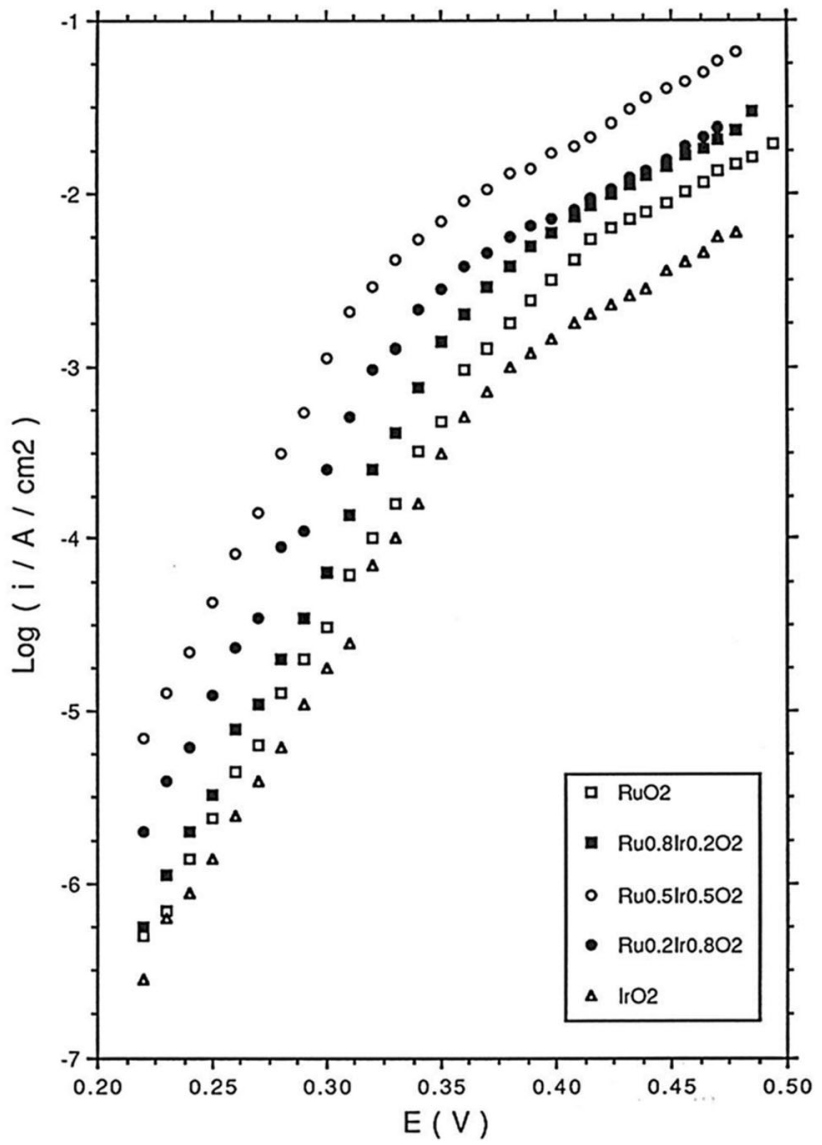

Fig. 18 Steady-state Tafel plots recorded in $1.0 \mathrm{M} \mathrm{NaOH}$ at $293 \mathrm{~K}$ for thermally prepared $\mathrm{RuO}_{2}, \mathrm{IrO}_{2}$ and mixed $\mathrm{Ru}_{x} \mid \mathrm{r}_{1-x} \mathrm{O}_{2}$ oxide coated Ti electrodes.

Experimentally, steady-state Tafel plots may be generated using a point by point method, performed using a series of small amplitude potential steps, e.g. $10 \mathrm{mV}$, and recording the steady state current after each step. Alternatively, linear sweep voltammetry performed at a slow sweep rate $\left(<10 \mathrm{mV} \mathrm{s}^{-1}\right)$ may be used to generate the current-potential profile. Typical Tafel plots recorded in $1.0 \mathrm{M} \mathrm{NaOH}$ for thermally prepared $\mathrm{IrO}_{2}, \mathrm{RuO}_{2}$ and mixed $\mathrm{Ru}_{x} \mathrm{Ir}_{1-x} \mathrm{O}_{2}$ oxide electrode materials are presented in Fig. 18. Similarly, sample plots recorded for electrochemically prepared hydrous Fe oxide and electroprecipitated $\mathrm{Ni}(\mathrm{OH})_{2}$ covered electrodes are presented in Fig. 19 and 20 respectively.

While a comprehensive review of the Tafel characteristics of the various metal oxide electrodes is beyond the scope of the current discussion, we wish to highlight here a number of general features relating to the Tafel analysis of these oxide systems. Firstly, it should be pointed out that the Tafel slopes recorded for the various oxides are often quite sensitive to factors such as the experimental conditions, the manner in which the oxides are prepared and even the experimental history of the electrode. For example, Lyons and Brandon ${ }^{14-16,281}$ have reported steady increases in Tafel slope from $c a .40 \mathrm{mV} \mathrm{dec}^{-1}$ to ca. $60 \mathrm{mV} \mathrm{dec}{ }^{-1}$ with extended use for passive oxide covered Fe and Co electrodes. These authors noted that the former Tafel slope is characteristic of a fresh electrode, whereas the latter value can be observed for an electrode that has undergone a
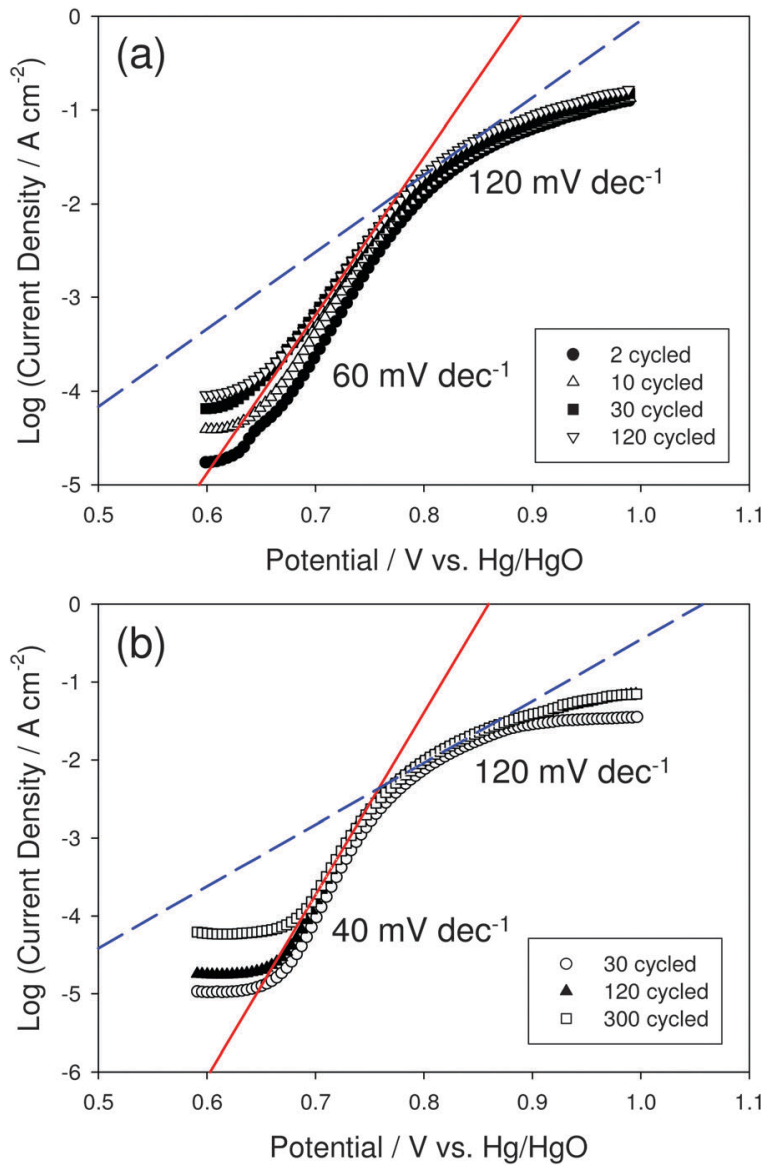

Fig. 19 Steady-state Tafel plots recorded in $1.0 \mathrm{M} \mathrm{NaOH}$ at $293 \mathrm{~K}$ for hydrous iron oxide films prepared using a range of growth cycles. The films were grown (a) in $1.0 \mathrm{M} \mathrm{NaOH}$ between switching potentials of $-1.45 \mathrm{~V}$ and $0.35 \mathrm{~V}$ at a sweep rate of $350 \mathrm{mV} \mathrm{s}^{-1}$ and (b) in $0.5 \mathrm{M} \mathrm{NaOH}$ between switching potentials of $-1.30 \mathrm{~V}$ and $0.75 \mathrm{~V}$ at a sweep rate of $400 \mathrm{mV} \mathrm{s}^{-1}$.

significant number of polarisation experiments. More recently, Doyle and Lyons ${ }^{96,98,99}$ have shown that the concentration of $\mathrm{NaOH}$ used in the preparation of hydrous Fe oxide layers has a significant influence on the observed Tafel slope for these electrode systems. It was observed that hydrous layers grown in high base concentrations had an associated Tafel slope of $c a$. $60 \mathrm{mV} \mathrm{dec}{ }^{-1}$ whereas those grown in low base concentrations $(<1.0 \mathrm{M})$ exhibited a Tafel slope of $c a .40 \mathrm{mV} \mathrm{dec}{ }^{-1}$. This differing Tafel behaviour, which has been labelled Type A and Type B respectively, ${ }^{96,98,99}$ has been attributed to dehydration of the hydrous layers prepared in the more concentrated base solutions. Similarly, as shown in Fig. 20, Lyons and co-workers ${ }^{167}$ have observed a steady decrease in Tafel slope from values in excess of $60 \mathrm{mV} \mathrm{dec}{ }^{-1}$ to $c a .45 \mathrm{mV} \mathrm{dec}{ }^{-1}$ with increasing solution concentration of $\mathrm{NaOH}$ for electroprecipitated $\mathrm{Ni}(\mathrm{OH})_{2}$ coated gold electrodes. This result was also interpreted in terms of a dehydration effect. It has been shown that the anhydrous $\beta$-form of $\mathrm{Ni}(\mathrm{OH})_{2}$ is more electrocatalytically active than the more hydrated $\alpha$-form. ${ }^{226}$ Indeed, it has been noted that $\beta$-NiOOH is the 'right type of oxide' for the OER. ${ }^{137}$ Thus, the increasing electrocatalytic behaviour of the $\mathrm{Ni}(\mathrm{OH})_{2}$ layer, as evidenced by the 

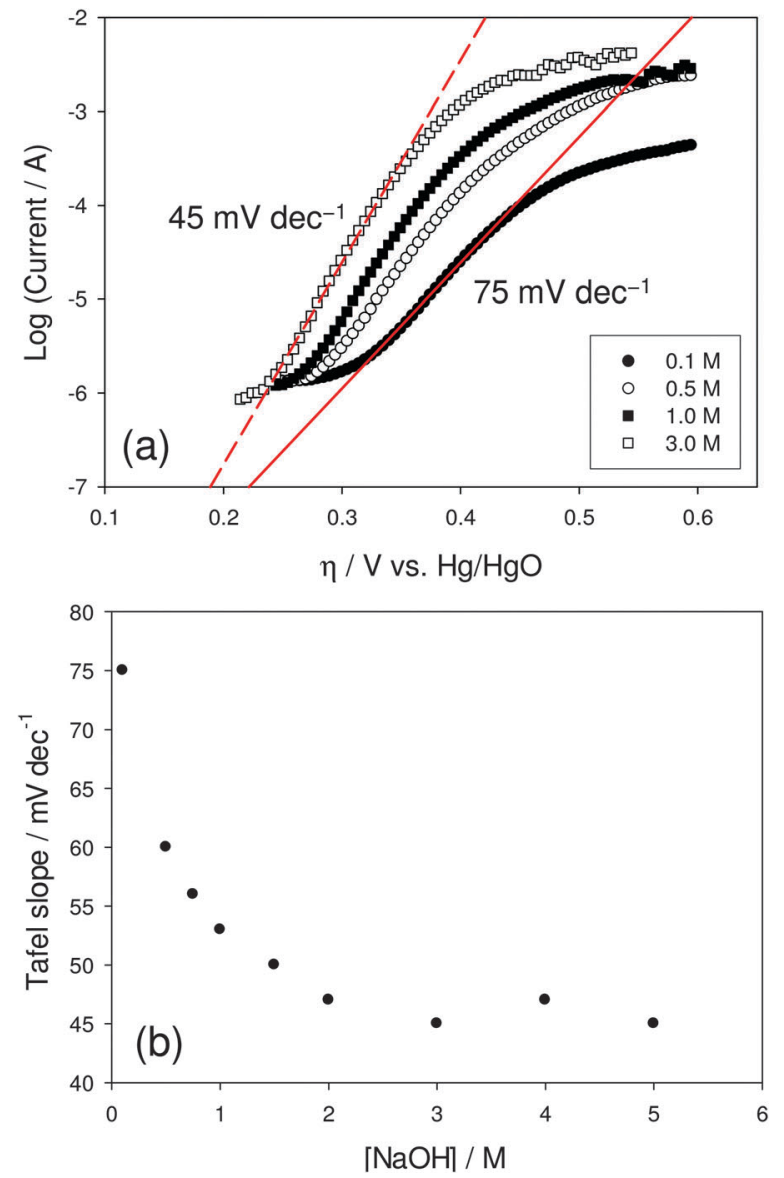

Fig. 20 (a) Steady-state Tafel plots recorded in a range of base concentrations for electroprecipitated $\mathrm{Ni}(\mathrm{OH})_{2}$ coated gold electrodes. (b) The experimental Tafel slope $b$ as a function of the solution $\mathrm{NaOH}$ concentration.

decrease in the Tafel slope with increasing base concentration, was attributed to higher levels of the $\beta$-form of the layer being present in the more concentrated base solutions. Conversely, in the case of thermally prepared $\mathrm{RuO}_{2}$ and $\mathrm{IrO}_{2}$ based electrodes

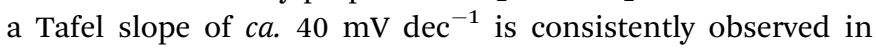
the literature. ${ }^{57,75,181}$ That said, some authors have observed variations in the slope depending on the surface roughness of the oxide layer. ${ }^{64,74}$

Secondly, an important kinetic feature of the OER Tafel plots presented here is the presence of two distinct linear regions. Such changes in Tafel slope with increasing overpotential are often observed in polarisation measurements that characterise the kinetics of the OER, and are particularly evident for the $\mathrm{RuO}_{2}$ and $\mathrm{IrO}_{2}$ plots in Fig. 18. Four possible explanations for the change in Tafel slope are often considered: ${ }^{276,280}$ (a) a change in reaction pathway, (b) a change in the electrode substrate, (c) a change in the RDS within a given pathway, and (d) the influence of changing potential on the adsorption of the reaction intermediates. However, it is the latter two reasons that are generally thought to be the most likely. Moreover, Damjanovic et al. ${ }^{276,280}$ suggest that it might be possible to discriminate between changes in Tafel behaviour due to either (c) or (d) by carefully examining the steady state polarisation curves. For example, they provide a rough calculation which shows that, if the heat of adsorption of an intermediate species changes by a typical value of about $10 \mathrm{Kcal} \mathrm{mol}^{-1}$ as its fractional coverage $\theta$ increases from 0.1 to 0.9 , then the range of potentials over which Temkin conditions might be expected to prevail is limited to $c a .150 \mathrm{mV}$. Thus, the limitation of one of the straight line Tafel regions to such a small potential range would point towards (d) as opposed to (c) being the reason behind the dual slope behaviour. That said, it is important to note that increases in the Tafel slope may not necessarily be mechanistically significant. A continuous increase in the Tafel slope with applied potential, as is apparent at very high overpotentials in Fig. 19 for the hydrous Fe oxide electrodes, may simply be the result of a reduction in the effective electrode surface area with increasing gas evolution at the higher applied potentials. Therefore, caution should be taken when interpreting multiple Tafel regions in terms of a possible mechanistic pathway.

In this respect, Trasatti et al. ${ }^{181,282}$ have outlined a useful method of Tafel analysis which can be used to separate ohmic drop effects from second Tafel slope effects, thereby allowing the identification of true mechanistically significant dual Tafel behaviour. In this method, the Tafel equation is modified to include the uncompensated ohmic drop as follows,

$$
E=a+b \log i+i R
$$

Differentiation of eqn (73) with respect to $i$ gives,

$$
\frac{\mathrm{d} E}{\mathrm{~d} i}=\frac{b}{i}+R
$$

Now it can be noted from eqn (74) that a plot of $\Delta E / \Delta i$ as a function of $1 / i$, where $\Delta E$ and $\Delta i$ are the change in potential and current for two consecutive data points respectively, and $i$ is the mean current for the same two data points, will be linear with slope $b$ equivalent to the Tafel slope and intercept $R$, which the latter authors have interpreted as the average uncompensated resistance between the electrode surface and the tip of the Luggin capillary or reference electrode over the explored potential range. The effectiveness of this type of data treatment is highlighted in Fig. 21 where a comparison of both types of Tafel analysis is shown. Steady-state polarisation data obtained for a hydrous oxide coated Fe electrode analysed in the usual manner is presented in Fig. 21a, while the same data analysed using eqn (74) is presented in Fig. 21b. Both methods gave virtually identical lower Tafel slopes. Furthermore, while there was slight disagreement regarding the upper Tafel slope with values of 118 and $100 \mathrm{mV} \mathrm{dec}{ }^{-1}$ obtained respectively, the important point here is that a clear and distinct second Tafel region was observed in both cases. Thus, in this situation, it could be concluded that the dual Tafel behaviour was in fact mechanistically significant and not simply due to ohmic effects.

An interesting feature of the latter type of data treatment is that it purports to offer an estimation of the uncompensated solution resistance $R_{\mathrm{u}}$. That is, the resistance between the reference probe electrode and the working electrode which gives rise to ohmic drop effects according to,

$$
E_{\text {eff }}=E_{\text {app }}-i R_{\mathrm{u}}
$$



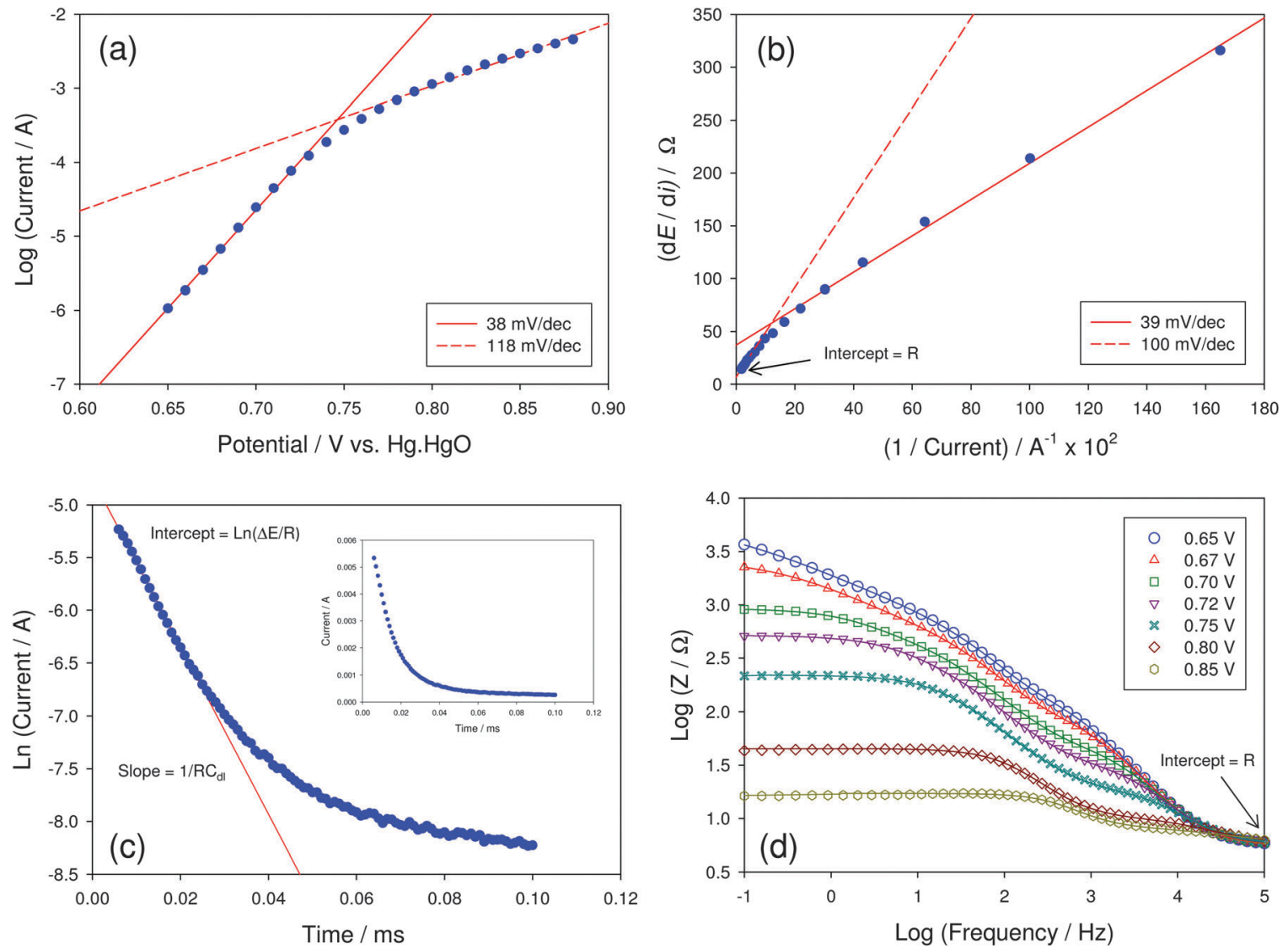

Fig. 21 (a) Steady-state Tafel plot recorded in $1.0 \mathrm{M} \mathrm{NaOH}$ at $298 \mathrm{~K}$ for a hydrous Fe oxide electrode prepared using 120 growth cycles. (b) The polarization data from (a) presented in the form of eqn (74). (c) A plot of In I vs. $t$ generated from the current-time transient (inset) resulting from a potential step from $0.50 \mathrm{~V}$ to $0.55 \mathrm{~V}$ vs. Hg/ $\mathrm{HgO}$ for a hydrous Fe oxide electrode. (d) A series of impedance spectra performed at various potentials associated with active oxygen evolution presented in the Bode-Log|Z| format for a hydrous Fe oxide electrode.

where $E_{\text {eff }}$ is the actual effective potential at the working electrode for an anodic reaction and $E_{\text {app }}$ is the potential applied by the potentiostat. Depending on the experimental conditions and electrochemical setup the effect of this resistance can be quite significant, particularly under high currents, and may need to be compensated for. Various methods of $i R$ compensation or elimination have been reported in the literature. Indeed this concept can be quite complex and has been the subject of a number of informative discussions, ${ }^{283,284}$ hence it will not be dealt with in detail here. However, it is worth noting that one of the simplest methods of $i R$ compensation involves the post-experimental correction of the data according to eqn (75) using an experimentally determined value of $R_{\mathrm{u}}$. In this manner, accurate $i R$ compensation relies on the accurate determination of $R_{\mathrm{u}}$.

Taking this into consideration, we have compared the value of $R_{\mathrm{u}}$ obtained using the method outlined by Trasatti et al. ${ }^{181,282}$ with those obtained using more established techniques in Table 1. Probably the simplest method involves the application of a small amplitude potential step in a potential region where no Faradaic processes are occurring, as shown in Fig. 21c. Under these conditions the only current flow should
Table 1 Comparison of the uncompensated solution resistance $R_{\mathrm{u}}$ values determined using different experimental and computational methods

\begin{tabular}{ll}
\hline Experimental method & Calculated $R_{\mathrm{u}} / \Omega$ \\
\hline Trasatti-Tafel & 6.4 \\
Potential step & 5.9 \\
EIS & 5.4 \\
CH iR test & 5.7
\end{tabular}

be due to double layer charging and the current response can be represented by, ${ }^{274}$

$$
i=\frac{\Delta E}{R_{\mathrm{u}}} \exp \left(\frac{-t}{R_{\mathrm{u}} C_{\mathrm{dl}}}\right)
$$

where $\Delta E$ is the magnitude of the potential step, typically $50 \mathrm{mV}$, and $C_{\mathrm{dl}}$ is the double layer capacitance. Furthermore, logarithmic analysis of eqn (76) gives,

$$
\ln i=\ln \left[\frac{\Delta E}{R_{\mathrm{u}}}\right]-\frac{-t}{R_{\mathrm{u}} C_{\mathrm{dl}}}
$$

implying that $R_{\mathrm{u}}$ can be calculated from the intercept of a plot of $\ln i$ as a function of the time $t$ (Fig. 21c). The obvious 
advantage of this method apart from its simplicity is the fact that it also allows for an estimation of the double layer capacitance from the slope of the $\ln i v s$. $t$ plot. Alternatively, the resistance $R_{\mathrm{u}}$ may be determined using EIS performed at a fixed potential, typically the open circuit potential. Although the impedance response of the electrochemical cell, when taken over a range of frequencies, will likely contain contributions from various diffusive, Faradaic and charging processes, at high frequencies these contributions are negligible compared with $R_{\mathrm{u}} \cdot{ }^{274}$ Consequently, $R_{\mathrm{u}}$ can be obtained from the high frequency resistive plateau in the Bode-Log $|Z|$ representation of the impedance data, as shown in Fig. 21d, or equivalently from the high frequency intercept in a Nyquist plot. However, if too high a frequency is used there is a danger that the measured impedance may no longer apply to the electrochemical cell but will be more representative of the electrical setup such as the wires, connections, etc. Therefore, it is recommended that an initial scan be performed over an extended frequency range to determine the most appropriate frequencies for $R_{\mathrm{u}}$ determination. Subsequent measurements may then be performed over an abbreviated frequency range or even at a single frequency. The final method employed was the built in $i R$ test function used on all $\mathrm{CH}$ potentiostat models. This method utilizes the same theory outlined in eqn (76) except that the current is sampled at set points after the potential step is applied and the initial current $I_{0}$ is calculated by extrapolating exponentially back to zero time. Now, since $\Delta E=I_{0} R_{\mathrm{u}}, R_{\mathrm{u}}$ can easily be calculated from this measurement and to reduce any error the software performs this procedure multiple times, outputting the average result. It can be seen from Table 1 that the values obtained for $R_{\mathrm{u}}$ using all four methods are in good agreement with each other with a mean value of $5.9 \pm 0.4 \Omega$. In particular, the value of $R_{\mathrm{u}}$ calculated from the Trasatti-Tafel analysis does not deviate significantly from the values obtained using the more established methods. In view of this, we believe Trasatti et al. ${ }^{181,282}$ have outlined a relatively simple yet powerful analytical tool for the interpretation of steady-state polarisation data, enabling a clear distinction between differing Tafel regions and yielding accurate estimates for the Tafel slopes and the uncompensated solution resistance $R_{\mathrm{u}}$.

Finally, further useful kinetic information can be obtained by performing steady-state Tafel analysis over a range of concentrations of a mechanistically significant reactant. In this way, apparent reaction orders $m$ may be determined from the slopes of $\log i v s$. $\log c$ plots at constant potentials, where $c$ is a concentration term. The evaluation of such reaction orders, when combined with corresponding Tafel slopes, helps to provide a comprehensive kinetic analysis of the reaction in question and is particularly useful in distinguishing between different possible mechanistic pathways. These experimentally determined reaction order values can often be potential dependent and so it is necessary to generate reaction order plots at a range of potentials. This is especially important for systems exhibiting dual Tafel behaviour, as distinct reaction orders can often be associated with each Tafel region.

In the case of alkaline water electrolysis the pertinent concentration term is the activity of the hydroxide ion $a_{\mathrm{OH}^{-}}$.
Typical reaction order plots for hydrous Fe oxide films prepared in a range of base concentrations are presented in Fig. 22, along with the corresponding series of steady-state Tafel plots. The $\mathrm{OH}^{-}$ion activities were calculated from the mean ionic activity coefficients $\gamma \pm$ reported in the literature. ${ }^{285}$ Reaction orders $m_{\mathrm{OH}^{-}}$of ca. $0.98 \pm 0.15,1.33 \pm 0.12,1.01 \pm 0.12$ and $0.92 \pm$ 0.04 were obtained at potentials located in the low Tafel region for hydrous Fe oxide films prepared in 0.1, 0.5, 1.0 and $5.0 \mathrm{M}$ $\mathrm{NaOH}$ respectively. In the high Tafel region the corresponding reaction orders were $0.62 \pm 0.05,0.95 \pm 0.18,0.94 \pm 0.06$ and $0.77 \pm 0.10$ respectively. Similar numerical values for the reaction orders were obtained for hydrous oxide layers prepared using a range of growth cycles indicating that the reaction order is not dependent on the hydrous oxide charge capacity.

The results outlined here highlight an important point which must be considered when interpreting reaction order data. That is, reaction orders should theoretically be integer values. While many of the latter reaction orders are statistically close to unity, some are of a more fractional nature. In a conventional kinetic analysis, under assumed Langmuir adsorption conditions with low surface coverage of intermediates, a reaction order $m_{\mathrm{OH}^{-}}=1$ suggests that only a single hydroxide ion reacts at each active site for all steps up to and including the RDS. Similarly, if $m_{\mathrm{OH}^{-}}=2$ then a total of $2 \mathrm{OH}^{-}$ equivalents can be assumed to be involved in the overall reaction prior to and including the RDS. However, it can be more difficult to rationalise fractional reaction orders. A number of authors have considered the effect of the diffuse layer potential $\phi^{*}$ on the observed kinetic parameters. ${ }^{75,286-288}$ Here, the diffuse layer corrected electrode potential is represented by $E-\phi^{*}$ where,

$$
\phi^{*}=\frac{R T}{F} \ln a_{\mathrm{s}}
$$

and $a_{\mathrm{s}}=a_{\mathrm{OH}^{-}}$or $a_{\mathrm{H}^{+}}$depending on the medium. Thus, if the experiments are not performed at a constant ionic strength then the observed reaction order might not correspond to a constant $E-\phi^{*}$. For example, Lyons and Floquet ${ }^{75}$ suggest that correcting for the diffuse layer potential can account for the fractional reaction order $m_{\mathrm{H}^{+}}=-1.5$ observed for $\mathrm{RuO}_{2}$ layers in acidic media. On the other hand, Carugati et $a l^{74}$ have proposed that fractional reaction orders may arise due to competing or parallel reaction pathways. In such cases, the experimentally observed kinetic parameters represent a combination of the parameters expected for the competing pathways, the exact value of which depends on the fraction of catalytic sites following each individual pathway. Then again, Lyons and Brandon ${ }^{92}$ have shown that the fractional reaction order $m_{\mathrm{OH}^{-}}=1.5$ observed for an 'aged' passive oxide coated iron electrode can be rationalised by admitting Temkin rather than Langmuir adsorption conditions.

(b) Open circuit potential decay. Steady-state measurements of electrode processes are often complemented by studies of the decay of the open circuit potential (OCP), which can also characterize in many cases the kinetics and mechanism of the basic process involved in the electrode reaction. This approach is frequently adopted in cases where elucidation of the reaction mechanism by steady state polarization techniques is 

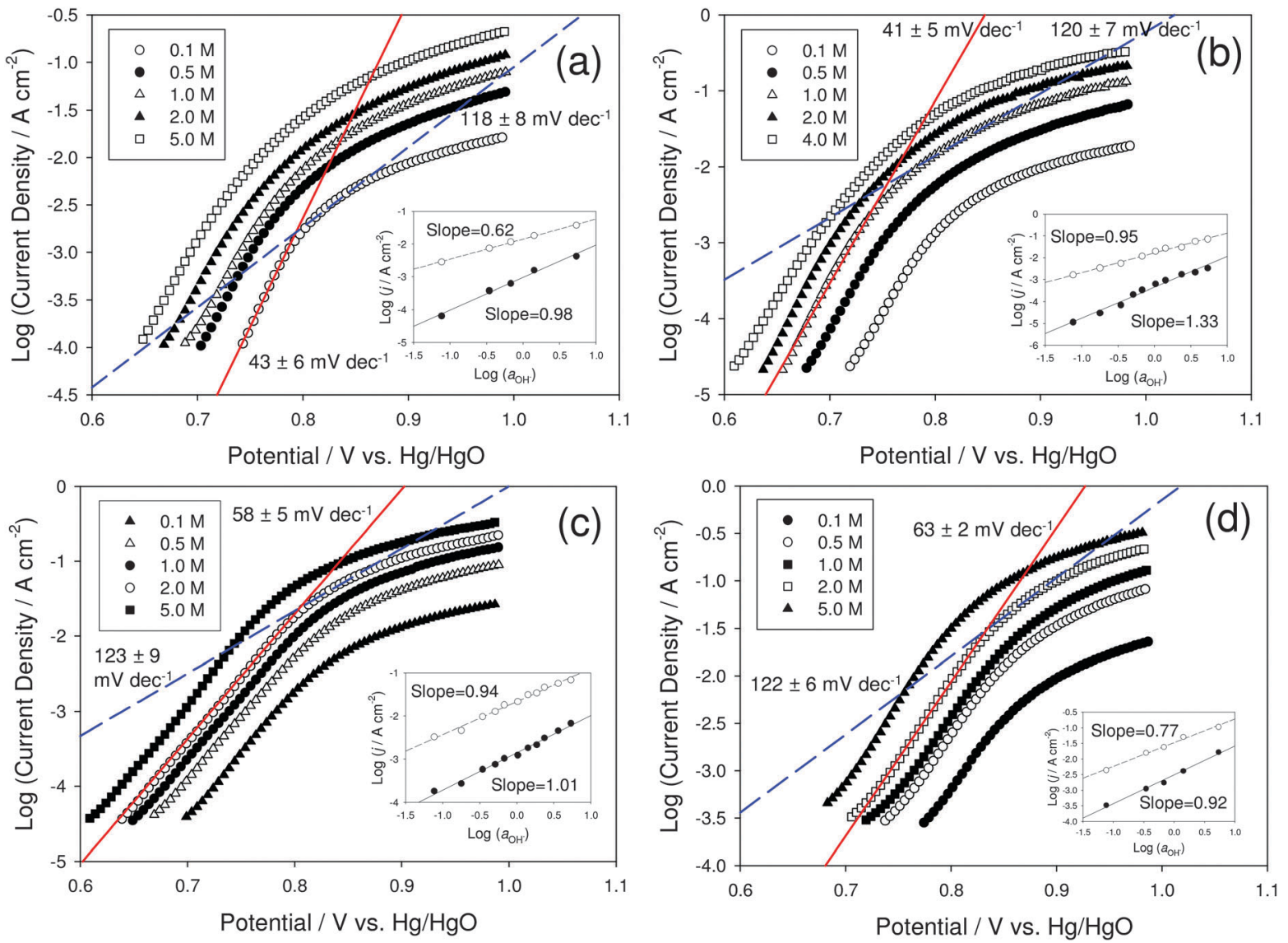

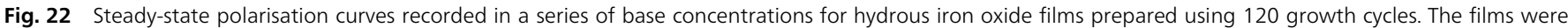

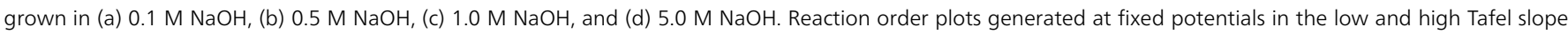
regions are shown in the insets of each graph.

complicated by continuous alteration of the electrode surface with time. Open circuit potential decay measurements are usually carried out by initially polarizing the electrode at a potential in the region where OER Tafel behaviour is observed. The duration of pre-OCP decay polarisation varies in the literature by both research group and the nature of the electrode but, generally the polarisation time should be sufficient to achieve a steady-state current. Most modern potentiostats now possess a pre-programmed OCP decay measurement function, allowing easy application of this technique. However, OCP decay measurements may also be obtained manually. In this method, the potentiostat is switched off after a suitable polarisation period and the electrode potential is measured at various time intervals using a high impedance voltmeter, which is disconnected between readings in order to avoid any forced discharge.

The basis of the study of open circuit potential decay curves is that the slope $-b_{\mathrm{OCP}}$ of the $E v s . \log t$ decay curve is equal to the slope $b$ of the Tafel plot for steady state polarization. ${ }^{289}$ Under open circuit conditions, the loss of charge capacity displayed by oxide films is thought to be a consequence of the fact that most of the charge storage region is located above the thermodynamic potential for the decomposition of water. Self-discharge then is assumed to proceed by an electrochemical mechanism analogous to that of corrosion. That is, the simultaneous occurrence of anodic and cathodic reactions as a mixed potential via a local cell mechanism.
In such systems either the anodic or cathodic process tends to be rate limiting and a potential corresponding to one or the other of the processes is set up. In the present case, self-discharge consists of a cathodic oxide reduction process and an anodic oxygen evolution process. It has been noted that the oxide reduction may be regarded as a combined electron and proton switching process in regions of the film where access by the electrolyte is low. ${ }^{57,75}$ Therefore, given the fast proton and electron switching transport properties of these films, as discussed in Section 4, the rate of self-discharge is assumed to be governed by the anodic oxygen evolution process.

In general, the current density at an electrode can be written as the sum of the charging current $i_{\mathrm{C}}$ and the Faradaic current $i_{\mathrm{F}}{ }^{289}$

$$
i=i_{\mathrm{C}}+i_{\mathrm{F}}
$$

At time $t_{0}$ when the current is switched off, $i=0$ and $\eta=\eta_{0}$. Hence,

$$
i_{\mathrm{C}}=-i_{\mathrm{F}}
$$

Noting that,

$$
i_{\mathrm{C}} \mathrm{d} t=\mathrm{d} q ; \quad C=\frac{\mathrm{d} q}{\mathrm{~d} V}=\frac{\mathrm{d} q}{\mathrm{~d} \eta_{\mathrm{t}}}
$$


where $q$ is charge and $C$ is capacitance, we can show that,

$$
-i_{\mathrm{F}} \mathrm{d} t=\mathrm{d} q=C \mathrm{~d} \eta_{\mathrm{t}}
$$

Under anodic conditions with $\eta_{\mathrm{t}}>R T / F$,

$$
-i_{0} \mathrm{e}^{\beta \eta_{\mathrm{t}} F / R T} \mathrm{~d} t=C \mathrm{~d} \eta_{\mathrm{t}}
$$

which after rearranging and integrating gives,

$$
\int_{t_{0}}^{t}\left(-i_{0}\right) \mathrm{d} t=\int_{\eta_{0}}^{\eta} C \mathrm{e}^{-\beta \eta_{\mathrm{t}} F / R T} \mathrm{~d} \eta_{\mathrm{t}}
$$

Now, for $t \gg t_{0}$ and $\eta_{0} \gg \eta$,

$$
-i_{0} t=\frac{R T}{\beta F} \mathrm{e}^{-\beta \eta_{\mathrm{t}} F / R T}
$$

and taking the logarithim gives,

$$
\eta_{\mathrm{t}}=\frac{2.303 R T}{\beta F} \log \left(\frac{R T}{\beta F i_{0}}\right)-\frac{2.303 R T}{\beta F} \log (t)
$$

Thus, an equivalent Tafel slope can be determined from the slope of a plot of the measured potential or overpotential during the decay as a function of $\log t$. Typical results for $\mathrm{RuO}_{2}$ and $\mathrm{IrO}_{2}$ are presented in Fig. 23, where well defined
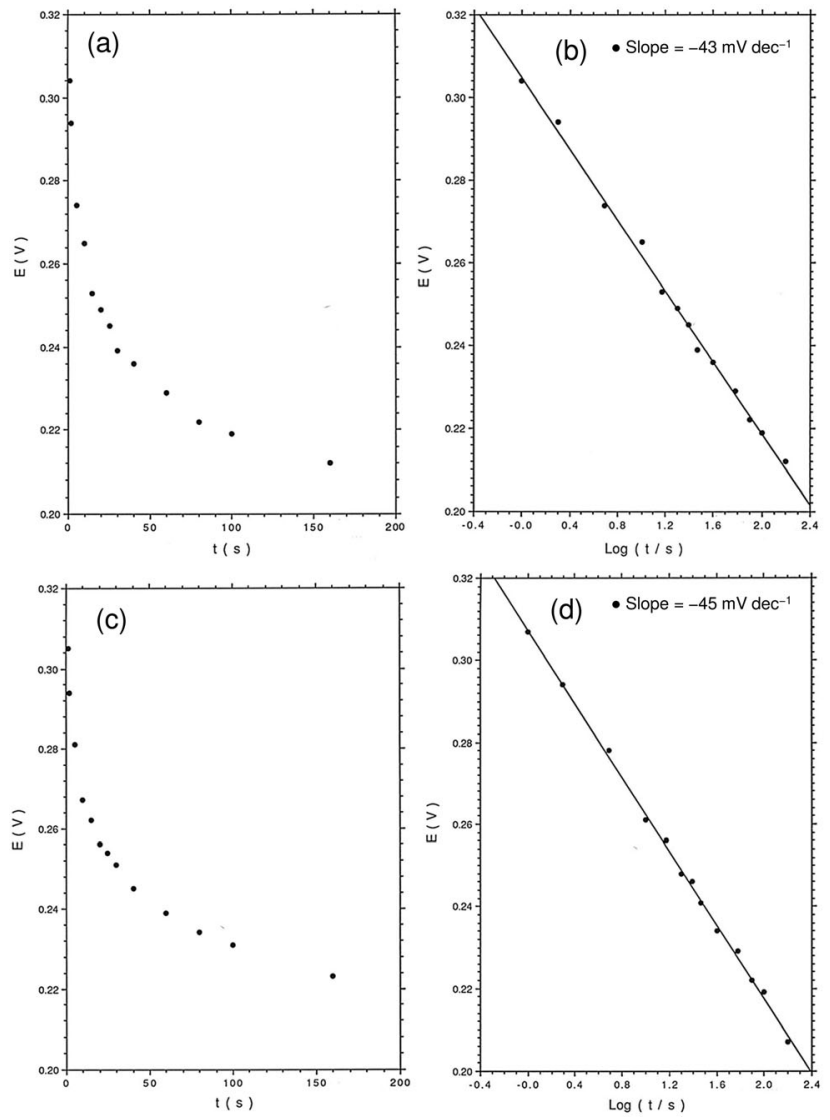

Fig. 23 Open circuit potential (OCP) decay curves recorded in $1.0 \mathrm{M} \mathrm{NaOH}$ for thermally prepared $\mathrm{RuO}_{2}(\mathrm{a}, \mathrm{b})$ and $\mathrm{IrO}_{2}(\mathrm{c}, \mathrm{d})$ films deposited on a Ti support. The variation of the OCP after a 5 min polarisation period at a potential in the oxygen evolution region is shown as a function of $(a, c)$ time and $(b, d) \log$ (time). exponential decays and excellent linear plots of $E v s$. $\log t$ can be observed. In this case, the slopes obtained from the $E v s . \log t$ curves were in good agreement with those derived from steady state polarization measurements ${ }^{75}$ highlighting the potential usefulness of this technique for the elucidation of Tafel slopes.

However, such simple relationships between the OCP decay slope and the steady-state Tafel slope are not always observed. This is evident in Fig. 24 where the OCP decay curves and corresponding $E v s$. $\log t$ plots for a hydrous oxide coated Fe electrode are shown. It is clear that more complicated OCP decay behaviour was recorded for the hydrous films. At short

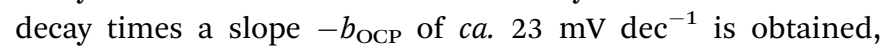
whereas at longer times the slope $-b_{\mathrm{OCP}}$ varies between $c a .160$ and $180 \mathrm{mV} \mathrm{dec}^{-1}$ depending on the initial polarisation potential. Importantly, these slopes differ considerably from the expected slope of $c a .60 \mathrm{mV} \mathrm{dec}{ }^{-1}$ which was obtained using steady-state polarisation and EIS techniques. ${ }^{96,98}$ Indeed, the presumption of numerical identity between the decay slope and the Tafel slope is not always justifiable. There is an assumption evident in the mathematical treatment outlined in eqn (79-86) that the capacitance $C$ is constant. $^{289}$ In fact, Conway and Bourgault ${ }^{290-292}$ have shown that interpretations of OCP decay slopes in terms of rate-determining mechanisms are only valid under conditions where the electrode surface capacity is independent of potential. In situations where the surface capacity varies during self-discharge the observed decay slope can differ quite significantly from the steady-state Tafel slope. In such cases, the latter authors have identified mathematical relationships between the observed decay slope and the true Tafel slope. In particular, two primary limiting cases have been discussed. ${ }^{290}$ If the surface coverage of intermediates during self-discharge is assumed to be low it can be shown that the surface capacity decreases with decreasing potential. Under these conditions the relationship between the decay slope $b_{\mathrm{OCP}}$ and the true Tafel slope $b$ is given by,

$$
b_{\mathrm{OCP}}=\frac{b^{\prime} b}{b-b^{\prime}}
$$

where $b^{\prime}$ is some positive multiple or fraction of $R T / F$. Hence the observed decay slope is predicted to be greater than the Tafel slope. Conversely, if there is high but not full coverage of intermediates the surface capacity increases with decreasing potential and the following expression for the decay slope is obtained,

$$
b_{\mathrm{OCP}}=-\frac{b^{\prime} b}{b^{\prime}-b}
$$

indicating that the observed decay slope will be less than the Tafel slope. In this way, Conway and Bourgault ${ }^{290}$ proposed that the relationship between the decay slope and the steady state Tafel slope could be used to determine the potential dependence of the surface capacity and the fractional surface coverage of adsorbed intermediates.

Taking this into consideration, the lack of agreement between the decay slopes and the Tafel slopes for the hydrous oxide layers suggests that the surface capacity does not remain 

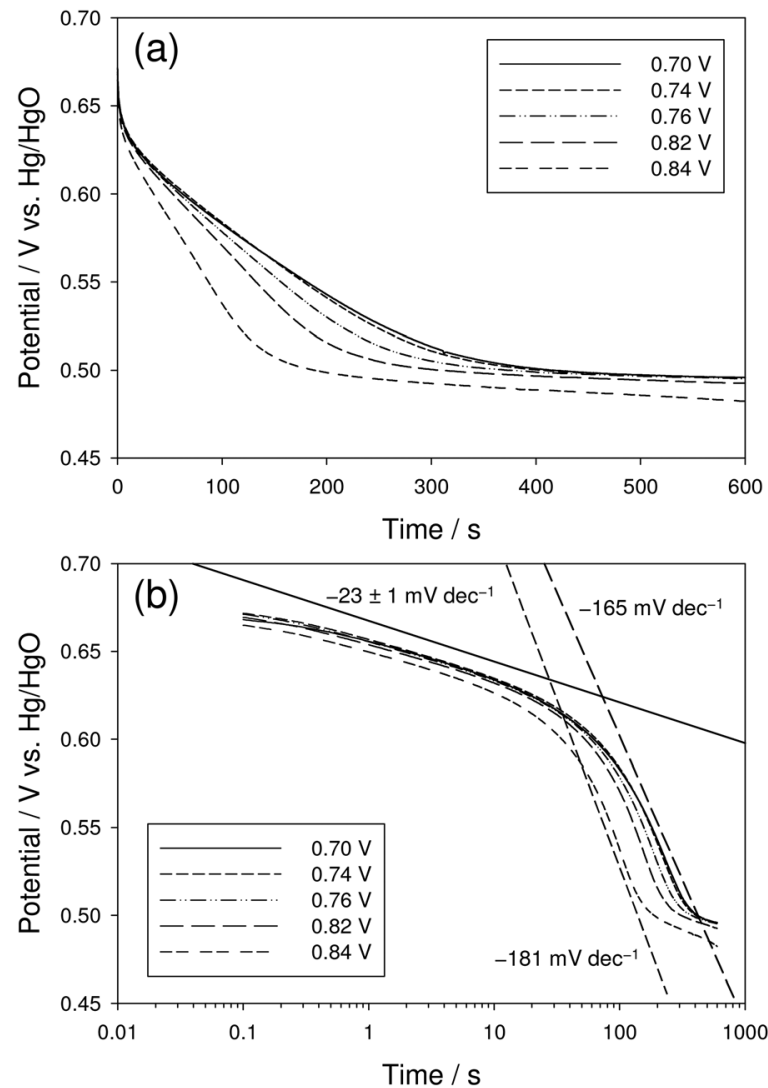

Fig. 24 Variation of OCP as a function of (a) time and (b) log (time) for a hydrous oxide coated Fe electrode in $1.0 \mathrm{M} \mathrm{NaOH}$ after a 5 min polarisation period at a series of potentials in the oxygen evolution region.

constant during self-discharge. Unlike the situation outlined by Conway and Bourgault, ${ }^{290}$ where the surface capacity was related to the surface coverage of discrete adsorbed intermediates, here the surface capacity is related to the concentration of charged surface or surfaquo groups. It is envisaged that these species are the active sites for oxygen evolution, becoming reduced as part of the accompanying cathodic process in self-discharge. In this respect, it is to be expected that the surface capacity varies during self-discharge or even during steady-state polarisation for that matter. In actual fact, Doyle and Lyons ${ }^{96,98}$ have shown previously that the double layer capacitance determined from EIS measurements is not constant during oxygen evolution, but increases with increasing potential in accordance with the formation of charged surface intermediates. Thus, it is possible that the variation in the decay slopes shown in Fig. 24 can be interpreted in terms of a decrease in the surface concentration of charged surfaquo groups. Following the reasoning of Conway and coworkers, at high potentials or short decay times a large proportion of the surfaquo groups are in the higher charged state implying significant surface coverage of intermediates and the observed decay slope is lower than the Tafel slope. On the other hand, for longer times the surface coverage becomes considerably reduced due to increased discharge of the surfaquo groups and the decay slope is greater than the Tafel slope. Of course this interpretation begs the question: why the seemingly constant surface capacity for the thermally prepared $\mathrm{RuO}_{2}$ and $\mathrm{IrO}_{2}$ films, as evidenced by the similarity of the slopes of the $E v s . \log t$ and Tafel plots. In this sense, it is instructive to note the extent of oxide reduction during the self-discharge process. In the case of the hydrous oxide films outlined above, the surfaquo group reduction encompasses the entire layer. However, for the thermally prepared films the reduction reaction does not proceed to any extent into the bulk of the film but remains essentially localized in the outer hydrated surface region. Hence, the reduction process which accompanies oxygen evolution during self-discharge may not significantly affect the surface capacity of the $\mathrm{RuO}_{2}$ and $\mathrm{IrO}_{2}$ films, thereby accounting for the similarity of the Tafel and OCP decay slopes.

(c) Electrochemical impedance spectroscopy. Electrochemical impedance spectroscopy (EIS) is a useful experimental tool in probing the kinetics of electrocatalytic reactions and in characterising the properties of the electrode/electrolyte interfaces at which such reactions occur. To measure the impedance of an electrochemical cell, a sinusoidal excitation voltage is applied to the system, over a range of frequencies, and the current response of the system is measured. In this way, electrochemical processes such as charge transfer, ion diffusion and capacitance are separated on the basis of their frequency dependent response and can be evaluated individually if their time constants differ enough, with fast processes being observed at high frequencies while slower processes are observed at lower frequencies. As was the case for the OCP decay measurements outlined above, an initial polarisation period at the applied potential is usually employed prior to recording the EIS response in order to allow the system to reach a steady-state. The analysis of EIS data recorded in this way often involves fitting to an electrical equivalent circuit which for our purposes can be developed from a mathematical analysis of the proposed OER mechanistic sequence. The components of the equivalent circuit, which may take the form of resistors, capacitors, inductors etc., can in many cases be assigned to physically meaningful parameters and processes such as rate constants via the Faradaic impedance, film resistances, and ionic and electronic transport rates. Thus, when the appropriate model is applied it can help to understand both the physicochemical properties of the oxide layer and the kinetics of the OER.

Lyons and coworkers have recently shown that the circuit in Fig. 25 is a good general model for the OER at passive oxide and hydrous oxide covered electrodes. ${ }^{96,98,293}$ Typical EIS responses for hydrous $\mathrm{Fe}$ oxide covered electrodes recorded over a range of potentials associated with active oxygen evolution are presented in Fig. 26. In general, the EIS responses of these types of electrode are characterised by three relaxation processes. This is clear from the three distinct capacitance peaks in the BodePhase plots of Fig. 26(3) and the three pseudo-semicircular regions at lower potentials in the Nyquist plots of Fig. 26(1). The $C_{\text {film }} R_{\text {film }}$ loop of the equivalent circuit model is observed at high frequencies and is attributed to the dielectric properties and the resistivity of the underlying compact oxide film, respectively. In some systems, particularly for the more conducting 


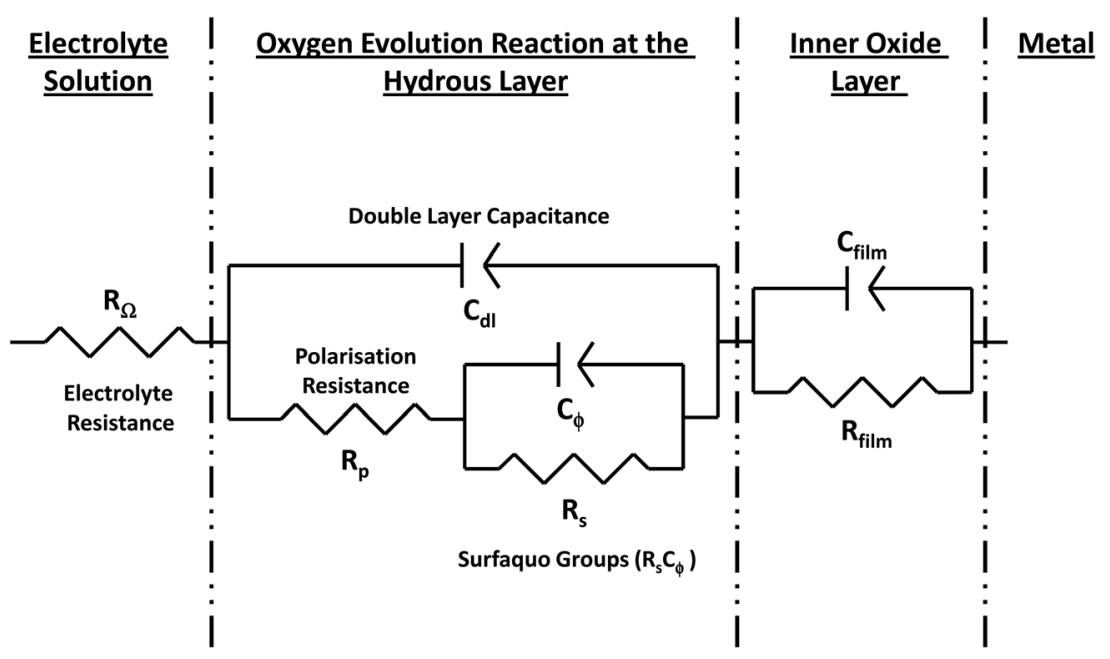

Fig. 25 Equivalent circuit used to model the OER at passive oxide and hydrous oxide coated transition metal electrodes.
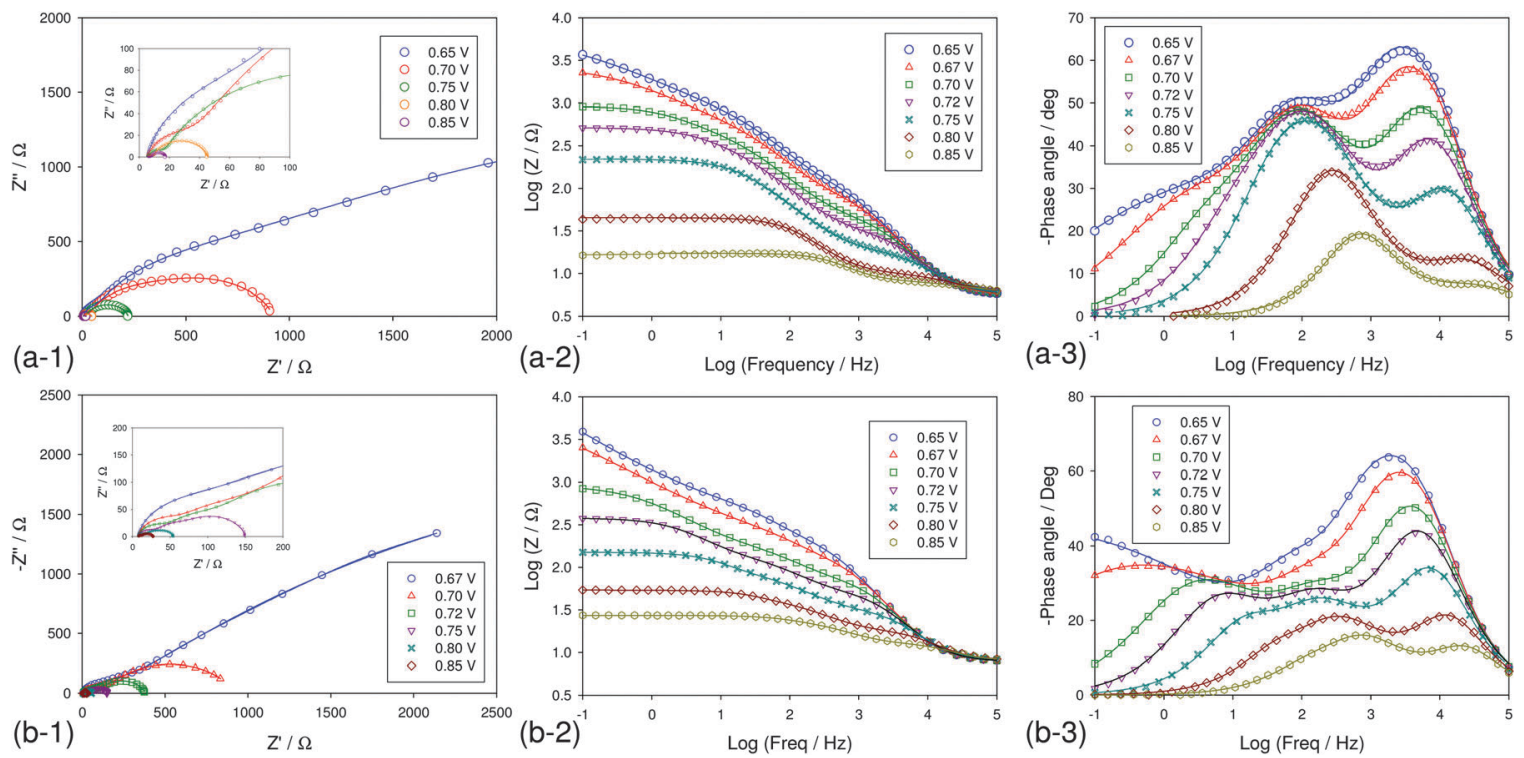

Fig. 26 Electrochemical impedance spectra recorded in $1.0 \mathrm{M} \mathrm{NaOH}$ at a series of potentials associated with active oxygen evolution for (a) Type A and (b) Type B 120 cycled hydrous iron oxide films in the Nyquist (1), Bode-Log $|Z|(2)$ and Bode-Phase format. The experimental data is represented by discrete points and the simulated impedance response generated using the equivalent circuit model in Fig. 25 is represented by a continuous line.

oxides such as $\mathrm{Ni}$ based systems, ${ }^{14,293}$ this feature can be difficult to observe due to a low film resistance/high electronic conductivity. The $C_{\mathrm{dl}}$ element models the double-layer capacitance, while $R_{\Omega}$ represents the electrolyte resistance. In terms of the electrocatalytic properties of the oxide layer, the resistive elements $R_{\mathrm{S}}$ and $R_{\mathrm{p}}$ are related to the kinetics of the interfacial charge transfer reaction. In particular, the polarisation resistance $R_{\mathrm{p}}$ can be viewed as a total charge transfer resistance for the combined steps, up to and including the RDS, of the OER. In the theoretical treatment of Harrington and Conway, ${ }^{294}$ the reciprocal resistance $1 / R_{\mathrm{p}}$ was shown to be equal to the sum of similar reciprocal resistances for each of the individual charge transfer steps. Consequently, $R_{\mathrm{p}}$ is related to the overall rate of the OER. On the other hand, the later authors have shown that
$R_{\mathrm{S}}$ is related, albeit in a rather complicated fashion, to the rate of production of one or more surface intermediates. ${ }^{294}$ In a general way, $R_{\mathrm{s}}$ gives an indication of the ease with which these species can be formed. Following the assertions of the same authors, $C_{\phi}$ is given the value of a capacitor which in parallel with $R_{\mathrm{S}}$ correctly models the relaxation of the charge associated with a surface intermediate.

Of particular interest here is the fact that both $C_{\mathrm{dl}}$ and $C_{\phi}$ have been shown to vary with potential in the oxygen evolution region. ${ }^{96,98}$ The fitting values obtained for these parameters are plotted as a function of applied potential in Fig. 27. It can be seen from Fig. 27a that the values of $C_{\mathrm{dl}}$ increased with increasing potential. Moreover, this increase in the capacitance coincided with the onset of significant oxygen evolution current 

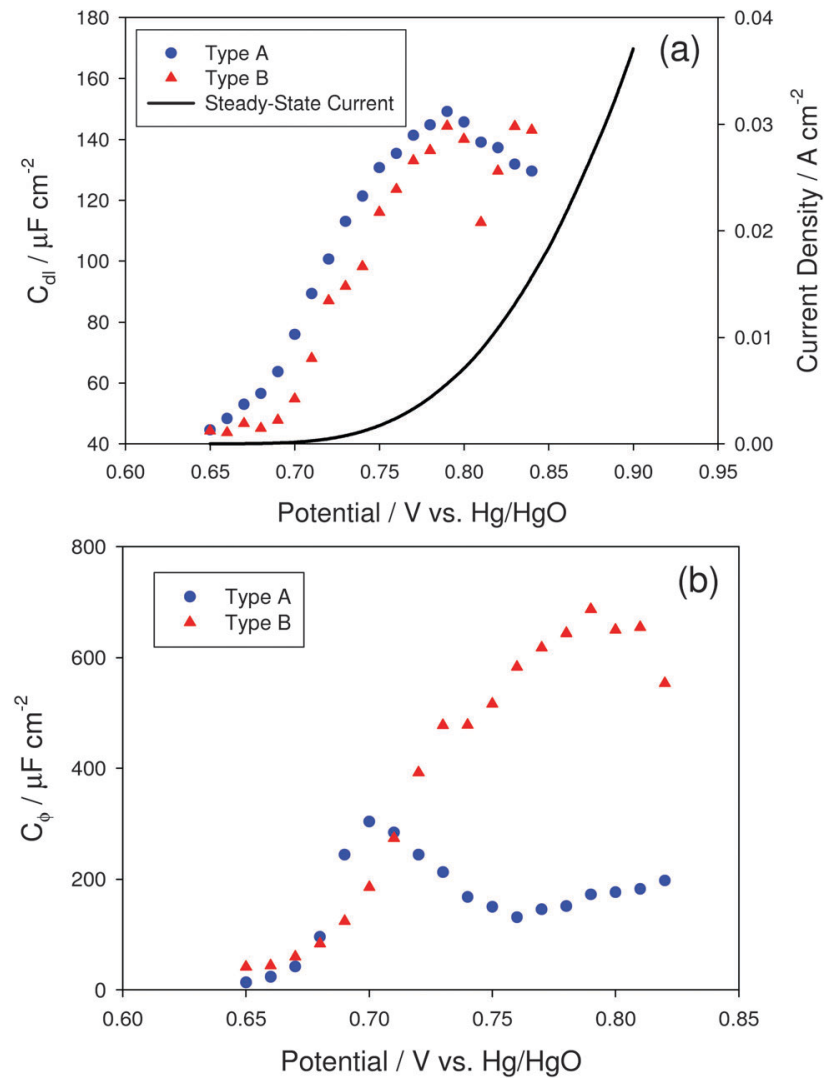

Fig. 27 The optimum fitting values of (a) $C_{d l}$ and (b) $C_{f}$ plotted as a function of applied potential for Type A and Type B 120 cycled hydrous oxide films. A typical steady state current profile for a 120 cycled Type A film is also presented in (a) above.

and therefore, is likely a reflexion of the increasing concentration of charged surface states as the OER progresses. Indeed, similar increases in the surface capacity due to the formation of surface states have been observed for thermally prepared $\mathrm{IrO}_{2}$ based electrodes, ${ }^{237}$ as well as hematite photoanodes. ${ }^{43,295}$ In this sense, the corresponding variation in $C_{\phi}$ with increasing OER potentials, which can be observed in Fig. 27b, has been attributed to the changing concentration of charged intermediates in the surface layer. In this way, the EIS results lend support to our interpretation of the OCP behaviour outlined above. That is, the lack of agreement between the Tafel slopes and the decay slopes arises from the potential dependence of the surface capacity of the hydrous oxide layers during oxygen evolution. Furthermore, the differing potential dependence of the $C_{\phi}$ element for Type A and Type B hydrous Fe oxide films in Fig. 27b suggests that this element describes a different charging process for each film. Thus, it has been proposed that different intermediates are involved in the OER rate-determining process for these films, in agreement with the contrasting Tafel behaviour observed for Type A and Type B hydrous oxide films. ${ }^{96,98}$

In addition to the latter type of interfacial analysis, EIS also provides a useful accessory method for the determination of Tafel slopes. Obtaining equivalent Tafel slopes using EIS involves the experimental determination of the total Faradaic resistance $R_{\mathrm{far}}$ as a function of applied potential. In the case of the equivalent circuit shown in Fig. 25, $R_{\text {far }}$ is calculated from the fitting parameters as $R_{\mathrm{far}}=R_{\mathrm{p}}+R_{\mathrm{s}}{ }^{92,96,98}$ At an oxygen evolution overpotential $\eta$ where simple Tafel behaviour prevails, the current density $i$ is related to $\eta$ via the following expression, ${ }^{92,96,98,296}$

$$
i=i_{0} \exp (2.303 \eta / b)
$$

where $i_{0}$ is the exchange current density for the OER. Taking the derivative of $i$ with respect to $\eta$ gives,

$$
\frac{\mathrm{d} i}{\mathrm{~d} \eta}=\frac{2.303 i}{b} \exp (2.303 \eta / b)
$$

Therefore, noting that $\mathrm{d} i / \mathrm{d} \eta=\mathrm{d} i / \mathrm{d} E=1 / R_{\text {far }}$, and by performing a logarithmic analysis of eqn (90) the following expression is achieved,

$$
\log \left(\frac{1}{R_{\mathrm{far}}}\right)=\frac{E}{b}+\log \left(\frac{2.303 i_{0}}{b}\right)
$$

implying that the inverse slope of a plot of $\log \left(1 / R_{\text {far }}\right)$ against $E$ is equal to the Tafel slope $b$. Sample plots of $\log \left(1 / R_{\text {far }}\right) v s$. E generated for the hydrous Fe oxide EIS data in Fig. 26 are presented in Fig. 28. For ease of comparison, the corresponding dc Tafel plots are also shown. Clear agreement is observed between the two different methods with Tafel slopes of
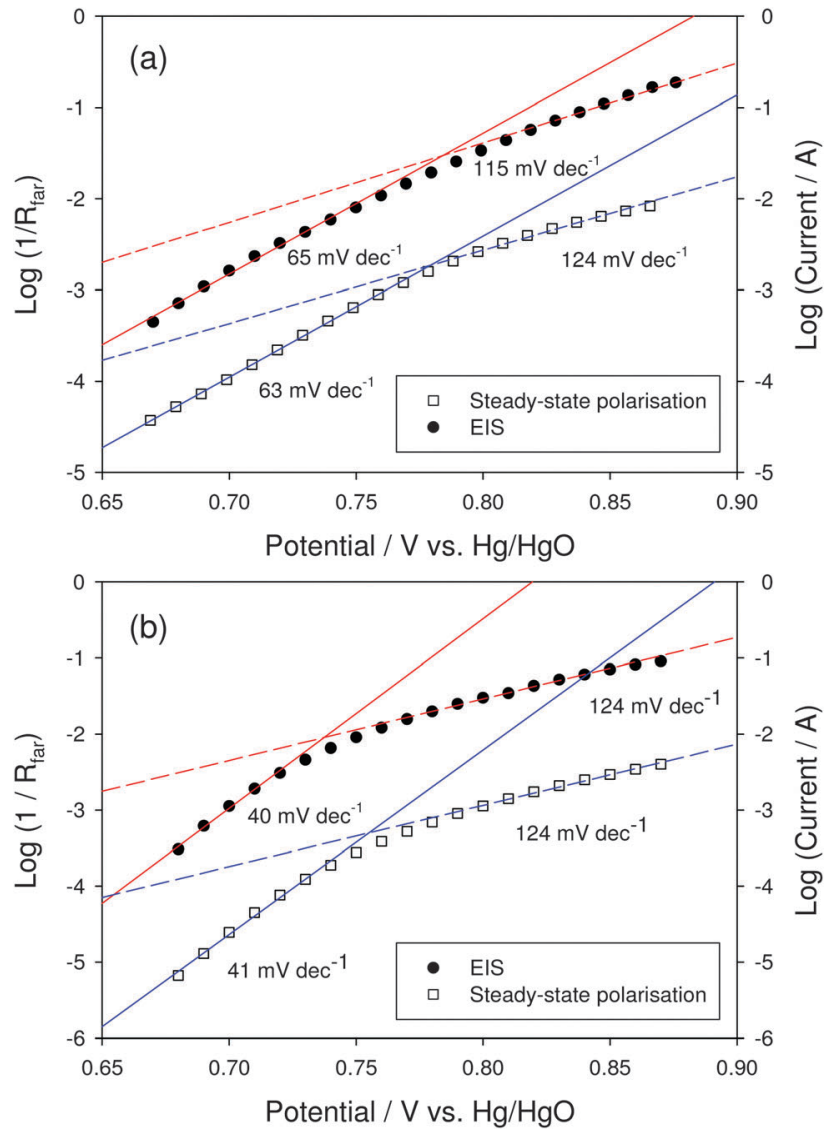

Fig. 28 Tafel plots generated from EIS and Steady-state polarisation data recorded in $1.0 \mathrm{M} \mathrm{NaOH}$ for (a) Type A and (b) Type B 120 cycled hydrous oxide films. 
ca. $60 \mathrm{mV} \mathrm{dec}{ }^{-1}$ and $40 \mathrm{mV} \mathrm{dec}^{-1}$ observed at low overpotentials and a Tafel slope of $c a .120 \mathrm{mV} \mathrm{dec}^{-1}$ uniformly observed at high overpotentials.

\subsection{Mechanistic studies of the OER}

In the face of the considerable research efforts devoted to the OER, the mechanism of the OER at transition metal oxide surfaces remains controversial. In terms of a mechanistic analysis, the major difficulty lies in the fact that the OER, as outlined in eqn (69), is a complex process involving the transfer of 4 electrons. Over the years various possible mechanistic schemes have been proposed. Indeed, if all possible reaction intermediates are taken into account a large number of viable pathways for the OER can be envisaged, ${ }^{297}$ although Hoare ${ }^{275}$ and Kinoshita ${ }^{298}$ both point out that by admitting a number of assumptions, the number of possible pathways can be reduced to ten or eleven. In Table 2 a summary of the diagnostic criteria for some of the most commonly considered oxygen evolution pathways is presented. Reproduced from the original work of Bockris and Otagawa, ${ }^{39,184}$ this table outlines the predicted Tafel slopes and reaction orders for each step in a given pathway. In this way, Table 2 highlights an inherent complication in attempts at kinetic mechanistic analysis of the OER. That is, the values of kinetic parameters such as Tafel slopes predicted for a given pathway are not unique. Hence, it is often very difficult to unambiguously identify the pathway operative for a particular system. In addition, the surface coverage of the reaction intermediates may well vary with potential, further complicating the thermodynamic and kinetic analysis of the OER. Thus, the important question of whether a common mechanism for the OER at transition metal oxides can be formulated, thereby facilitating a theory of electrocatalysis for oxygen evolution, is currently unresolved.

In light of this, much research has been focused on attempts to rationalise the relative electrocatalytic activities of different electrode materials in terms of a given physicochemical property. ${ }^{302}$ Indeed, considering the variety of OER anode materials in the literature the idea of such a universal 'descriptor' of catalytic activity is particularly attractive. However, attempts to draw correlations between electrode physicochemical properties and OER catalytic performance is complicated by the fact that it is the metal oxide and not the metal that catalyses oxygen evolution. In their seminal electrochemical work, Bockris and Ottagawa $^{39,184}$ suggested that the catalytic OER activity correlated inversely with the surface bond energy of $\mathrm{OH}$. Accordingly, the rate determining step may be the desorption of $\mathrm{OH}$ or oxygenated species from the surface - obviously the weaker the metal-OH bond the greater the rate of desorption at a given overpotential. More recently, theoretical calculations based on Density Functional Theory (DFT) and Molecular Orbital (MO) Theory have made significant contributions to the study of the OER. Rossmeisl et al. ${ }^{271}$ suggest that the surface oxygen binding energy is a suitable descriptor for OER activity with the formation of a surface adsorbed $\mathrm{OOH}$ species being ratedetermining at rutile-type oxides such as $\mathrm{RuO}_{2}, \mathrm{IrO}_{2}$ and $\mathrm{TiO}_{2}$. On the other hand, Suntivich et al. ${ }^{303}$ and Subbaraman et $a l .{ }^{304}$ have focused their catalytic analysis at a more atomic/ molecular scale. The former authors argue that the level of occupancy of the $e_{\mathrm{g}}$-symmetry electrons in the transition metal of a perovskite $\mathrm{AMO}_{3}$ is a suitable descriptor as it determines the binding of oxygen intermediates to the metal $\mathbf{M}$ whereas, the latter authors propose a correlation between the metalhydroxide bond strength in 3d-metal oxyhydroxide surface clusters and their OER catalytic activity.

Certainly the incumbent trend in mechanistic interpretations of the OER is toward a focus on the molecular rather than solid state properties of the anode materials. This is highlighted in the work of Suntivich ${ }^{303}$ and Subbaraman ${ }^{304}$ outlined above and concurrently through the dramatic rise in interest in the area of homogeneous water oxidation catalysis. Notable works include those of Meyer, ${ }^{305-308}$ Thummel, ${ }^{309-311}$ Llobet, $^{312-316}$ Sun,,$^{316-319}$ and Crabtree ${ }^{320-322}$ to name but a few. One of the advantages of these systems is the ease with which mechanistic studies can be performed. In this respect, it is interesting to observe that intermediate species similar to those proposed for heterogeneous systems, such as $\mathrm{M}-\mathrm{OH}, \mathrm{M}=\mathrm{O}$ and $\mathrm{M}-\mathrm{OOH}$ where $M$ is a catalytically active metal centre, are typically implicated for the molecular catalysts. ${ }^{323,324}$ Indeed, to facilitate a comparison of mechanisms it has been noted that the discharge of a hydroxide ion at an electrode surface, such as those outlined in Table 2, can be related to ligation of the hydroxide ion to a metal of the surface oxide, with a resultant increase of one in the formal oxidation state of the metal. ${ }^{10}$ Moreover, it has been suggested that bulk metal oxides represent a catalytic material which may share properties with both heterogeneous electrocatalysts and homogeneous molecular catalysts. In particular, Dau et al. ${ }^{10}$ pose the tantalising conceptual question of whether the more amorphous metal oxide/ hydroxide materials can be viewed best as an extended solidstate material or rather as an aggregate of multiple metal oxide complexes with molecular properties. Specifically, the latter authors note that, for a mechanistic discussion of the CoCF developed by Nocera and coworkers, ${ }^{26}$ it may not be necessary to extend the structure beyond the basic repeating unit. It is our opinion that this concept, bridging the fields of homogeneous and heterogeneous catalysis, holds the key to further advancement in the mechanistic understanding of the OER.

In the early mechanistic schemes outlined in Table 2, the OER was usually interpreted in terms of an initial discharge of hydroxide ions at the surface leading to the formation of discrete adsorbed intermediates such as $\mathrm{OH}$ radicals,

$$
\mathrm{M}+\mathrm{OH}^{-} \rightarrow \mathrm{MOH}+\mathrm{e}^{-}
$$

with subsequent steps in the reaction involving the formation of a range of surface adsorbed intermediates such as MO, MOOH or physisorbed peroxide species. ${ }^{10,11}$ These surface species could then react with each other, with other intermediates or with the electrolyte to liberate oxygen gas, the specific details of which depending on the various anode materials and experimental conditions. According to this classical adsorption type approach, if the stability of the adsorbed intermediate formed in the primary discharge step can be increased, then 
Table 2 Kinetic parameters derived for the 5 most common OER mechanistic pathways ${ }^{39,184}$

\begin{tabular}{|c|c|c|c|c|c|c|c|c|c|c|}
\hline \multirow[b]{3}{*}{ Rate-determining step } & \multicolumn{5}{|c|}{ Langmuir } & \multicolumn{5}{|c|}{ Temkin } \\
\hline & \multirow[b]{2}{*}{$v^{b}$} & \multicolumn{2}{|l|}{$\frac{\partial E^{a}}{\partial \ln i}$} & {$\left[\frac{\partial \ln i}{\partial \ln C_{\mathrm{OH}^{-}}}\right]$} & $E, c_{c}$ & \multicolumn{2}{|l|}{$\frac{\partial E}{\partial \ln i}^{a}$} & \multirow[b]{2}{*}{$\mathrm{NA}^{d}$} & \multirow[b]{2}{*}{$\mathrm{A}^{e}$} & \multirow[b]{2}{*}{ Condition $^{f}$} \\
\hline & & $\theta \rightarrow 0$ & $\theta \rightarrow 1$ & $\theta \rightarrow 0$ & $\theta \rightarrow 1$ & $\mathrm{NA}^{d}$ & $\mathrm{~A}^{e}$ & & & \\
\hline (I) Bockris's oxide path 39,184 & & & & & & & & & & \\
\hline 1. $\mathrm{M}+\mathrm{OH}^{-} \rightarrow \mathrm{MOH}+\mathrm{e}^{-}$ & 4 & $2 R T / F$ & & 1 & & & & & & \\
\hline 2. $2 \mathrm{MOH} \rightarrow \mathrm{MO}+\mathrm{M}+\mathrm{H}_{2} \mathrm{O}$ & 2 & $R T / 2 F$ & $\infty$ & 2 & 0 & $\begin{array}{l}2 R T / F \\
R T / F\end{array}$ & & $\begin{array}{l}0.5 \\
1\end{array}$ & & $\begin{array}{l}r_{\mathrm{OH}} \sim r_{\mathrm{O}} \\
r_{\mathrm{OH}} \gg r_{\mathrm{O}}\end{array}$ \\
\hline $\begin{array}{l}\text { 3. } 2 \mathrm{MO} \rightarrow 2 \mathrm{M}+\mathrm{O}_{2} \\
\text { (II) Bockris's electrochemical path } \\
39,184\end{array}$ & 1 & $R T / 4 F$ & $\infty$ & 4 & 0 & $\begin{array}{l}R T / 2 F \\
R T / 4 F\end{array}$ & $\begin{array}{l}R T / F \\
R T / 3 F\end{array}$ & $\begin{array}{l}2 \\
2\end{array}$ & $\begin{array}{l}1 \\
1\end{array}$ & $\begin{array}{l}K_{2} \sim 1 \\
K_{2} \ll 1\end{array}$ \\
\hline 1. $\mathrm{M}+\mathrm{OH}^{-} \rightarrow \mathrm{MOH}+\mathrm{e}^{-}$ & 2 & $2 R T / F$ & & 1 & & & & & & \\
\hline 2. $\mathrm{MOH}+\mathrm{OH}^{-} \rightarrow \mathrm{MO}+\mathrm{H}_{2} \mathrm{O}+\mathrm{e}^{-}$ & 2 & $2 R T / 3 F$ & $2 R T / F$ & 2 & 1 & $\begin{array}{l}2 R T / F \\
R T / F\end{array}$ & & $\begin{array}{l}1 \\
1.5\end{array}$ & & $\begin{array}{l}r_{\mathrm{OH}} \sim r_{\mathrm{O}} \\
r_{\mathrm{OH}} \gg r_{\mathrm{O}}\end{array}$ \\
\hline $\begin{array}{l}\text { 3. } 2 \mathrm{MO} \rightarrow 2 \mathrm{M}+\mathrm{O}_{2} \\
\text { (III) Krasil'shchikov's path }\end{array}$ & 1 & $R T / 4 F$ & $\infty$ & 4 & 0 & $\begin{array}{l}R T / 2 F \\
R T / 4 F\end{array}$ & $\begin{array}{l}R T / F \\
R T / 3 F\end{array}$ & $\begin{array}{l}2 \\
4\end{array}$ & $\begin{array}{l}1 \\
3\end{array}$ & $\begin{array}{l}K_{2} \sim 1 \\
K_{2} \ll 1\end{array}$ \\
\hline 1. $\mathrm{M}+\mathrm{OH}^{-} \rightarrow \mathrm{MOH}+\mathrm{e}^{-}$ & 2 & $2 R T / F$ & & 1 & & & & & & \\
\hline 2. $\mathrm{MOH}+\mathrm{OH}^{-} \rightarrow \mathrm{MO}^{-}+\mathrm{H}_{2} \mathrm{O}$ & 2 & $R T / F$ & $\infty$ & 2 & 1 & $\begin{array}{l}\infty \\
2 R T / F\end{array}$ & & $\begin{array}{l}1 \\
1.5\end{array}$ & & $\begin{array}{l}\mathrm{r}_{\mathrm{OH}} \sim r_{\mathrm{O}-} \\
\mathrm{r}_{\mathrm{OH}} \gg r_{\mathrm{O}-}\end{array}$ \\
\hline 3. $\mathrm{MO}^{-} \rightarrow \mathrm{MO}+\mathrm{e}^{-}$ & 2 & $2 R T / 3 F$ & $2 R T / F$ & 2 & 0 & $\begin{array}{l}2 R T / F \\
2 R T / F\end{array}$ & & $\begin{array}{l}0 \\
1\end{array}$ & & $\begin{array}{l}K_{2} \sim 1 \\
K_{2} \ll 1\end{array}$ \\
\hline 4. $2 \mathrm{MO} \rightarrow 2 \mathrm{M}+\mathrm{O}_{2}$ & 1 & $R T / 4 F$ & $\infty$ & 4 & 0 & $\begin{array}{l}R T / 2 F \\
R T / 4 F\end{array}$ & $\begin{array}{l}R T / F \\
R T / 3 F\end{array}$ & $\begin{array}{l}2 \\
2\end{array}$ & $\begin{array}{l}1 \\
1\end{array}$ & $\begin{array}{l}K_{3} \sim 1 \\
K_{3} \ll 1\end{array}$ \\
\hline $\begin{array}{l}\text { (IV) O'Grady's path }{ }^{300} \\
\text { 1. } \mathrm{M}^{z}+\mathrm{OH}^{-} \rightarrow \mathrm{M}^{z} \mathrm{OH}+\mathrm{e}^{-}\end{array}$ & 2 & $2 R T / F$ & & 1 & & & & & & \\
\hline 2. $\mathrm{M}^{z} \mathrm{OH} \rightarrow \mathrm{M}^{z+1} \mathrm{OH}+\mathrm{e}^{-}$ & 2 & $2 R T / 3 F$ & $2 R T / F$ & 1 & 0 & $\begin{array}{l}2 R T / F \\
R T / F\end{array}$ & & $\begin{array}{l}0 \\
0.5\end{array}$ & & $\begin{array}{l}\mathrm{r}_{1} \sim r_{2}^{g} \\
\mathrm{r}_{1} \gg r_{2}\end{array}$ \\
\hline $\begin{array}{l}\text { 3. } 2 \mathrm{M}^{z+1} \mathrm{OH}+2 \mathrm{OH}^{-} \rightarrow \mathrm{M}^{z}+\mathrm{H}_{2} \mathrm{O}+\mathrm{O}_{2} \\
\text { (V) Kobussen's path }\end{array}$ & 1 & $R T / 4 F$ & $\infty$ & 4 & 2 & $\begin{array}{l}R T / 2 F \\
R T / 4 F\end{array}$ & $\begin{array}{l}R T / F \\
R T / 3 F\end{array}$ & $\begin{array}{l}4 \\
4\end{array}$ & $\begin{array}{l}3 \\
3\end{array}$ & $\begin{array}{l}K_{2} \sim 1 \\
K_{2} \ll 1\end{array}$ \\
\hline 1. $\mathrm{M}+\mathrm{OH}^{-} \rightarrow \mathrm{MOH}+\mathrm{e}^{-}$ & 1 & $2 R T / F$ & & 1 & & & & & & \\
\hline 2. $\mathrm{MOH}+\mathrm{OH}^{-} \rightarrow \mathrm{MO}+\mathrm{H}_{2} \mathrm{O}+\mathrm{e}^{-}$ & 1 & $2 R T / 3 F$ & $2 R T / 3 F$ & 2 & 1 & $\begin{array}{l}2 R T / F \\
R T / F\end{array}$ & & $\begin{array}{l}1 \\
1.5\end{array}$ & & $\begin{array}{l}\mathrm{r}_{\mathrm{OH}} \sim r_{\mathrm{O}} \\
\mathrm{r}_{\mathrm{OH}} \gg r_{\mathrm{O}}\end{array}$ \\
\hline 3. $\mathrm{MO}+\mathrm{OH}^{-} \rightarrow \mathrm{MO}_{2} \mathrm{H}^{-}$ & 1 & $R T / 2 F$ & $\infty$ & 3 & 1 & $\begin{array}{l}\infty \\
R T / F\end{array}$ & & $\begin{array}{l}1 \\
2\end{array}$ & & $\begin{array}{l}K_{2} \sim 1 \\
K_{2} \ll 1\end{array}$ \\
\hline 4. $\mathrm{MO}_{2} \mathrm{H}^{-}+\mathrm{OH}^{-} \rightarrow \mathrm{MO}_{2}^{-}+\mathrm{H}_{2} \mathrm{O}+\mathrm{e}^{-}$ & 1 & $2 R T / 5 F$ & $2 R T / F$ & 4 & 1 & $\begin{array}{l}2 R T / F \\
2 R T / F\end{array}$ & & $\begin{array}{l}1 \\
2\end{array}$ & & $\begin{array}{l}K_{3} \sim 1 \\
K_{3} \ll 1\end{array}$ \\
\hline 5. $\mathrm{MO}_{2}^{-} \rightarrow \mathrm{M}+\mathrm{O}_{2}+\mathrm{e}^{-}$ & 1 & $2 R T / 7 F$ & $\infty$ & 4 & 0 & $\begin{array}{l}R T / F \\
R T / 2 F\end{array}$ & $\begin{array}{l}2 R T / F \\
2 R T / 3 F\end{array}$ & $\begin{array}{l}1 \\
1\end{array}$ & $\begin{array}{l}0.5 \\
1.5\end{array}$ & $\begin{array}{l}K_{4} \sim 1 \\
K_{4} \ll 1\end{array}$ \\
\hline
\end{tabular}

${ }^{a}$ Symmetry factors, i.e. $\beta, \gamma$, and $\delta$, in all steps, were taken as $\frac{1}{2} \cdot{ }^{b}$ Stoichiometric number. ${ }^{c} \zeta$ is the potential difference between the outer Helmholtz plane and the bulk of the solution. ${ }^{d}$ Nonactivated desorption of $\mathrm{O}_{2} .{ }^{e}$ Activated desorption of $\mathrm{O}_{2} \cdot{ }^{f} r$ is a coefficient determining the variation of heat of adsorption of a particular species with coverage. Unless stated, $r$ values for each species were taken as equal. $K_{i}$ is the equilibrium constant of the $i$ th step. ${ }^{g} r_{1}$ and $r_{2}$ refer to $r$ for $\mathrm{M}^{z} \mathrm{OH}$ and $r$ for $\mathrm{M}^{z+1} \mathrm{OH}$, respectively.

the rate control can shift to a later step. Thus, low Tafel slopes were attributed to the interaction of strongly adsorbed surface intermediates. ${ }^{275,325}$ An alternative view point is highlighted in the mechanism of $\mathrm{O}^{\prime} \mathrm{Grady}^{300}$ in Table 2, who emphasised the importance of oxide redox transitions in the course of the OER. In this often encountered concept, the OER is facilitated by the cyclic formation and decomposition of an unstable intermediate containing a metal centre in a higher valence state $(z+1)$ than its initial state $(z){ }^{300,326}$ The significance of this approach lies in its ability to account for low experimental Tafel slopes without the need to consider strong surface adsorption.

In the latter mechanistic schemes, the OER was not expected to occur on the entire available electrode surface but was instead localised at specific catalytically active surface sites, somewhat ambiguously represented by $M$. Indeed, Trasatti ${ }^{64}$ has noted that the origin of the electrocatalytic properties of thermally prepared $\mathrm{RuO}_{2}$ films is not to be sought in the intrinsic rutile structure of the oxide, rather the catalytic properties are more likely to originate from the state of surface atoms. In this sense, Lyons and co-workers ${ }^{96,98,281}$ suggest that the specific nature of the active site can be envisaged if attention is paid to the underlying surface chemistry. Importantly, negative super-Nernstian E-pH shifts have been observed for the oxides of a number of transition metals including $\mathrm{Ni},{ }^{167,211} \mathrm{Fe},{ }^{84,85,98}$ $\mathrm{Au},{ }^{175} \mathrm{Rh},{ }^{262} \mathrm{Ir}^{176}$ and $\mathrm{Ru} .{ }^{57,75}$ Recalling our discussion on the redox properties of the various oxides, such shifts in peak potential with increasing $\mathrm{pH}$ imply that the oxidised state has acquired a negative charge relative to the reduced state. This anionic oxide formation arises from the well known acidic properties of oxide surfaces in solutions of high $\mathrm{pH},{ }^{45,327}$ and can be equivalently regarded in terms of the adsorption of excess $\mathrm{OH}^{-}$ions, proton loss from coordinated water molecules or the formation of hydroxyl surface complexes. ${ }^{177,211}$ For example, O'Sullivan and Burke ${ }^{328}$ proposed, based on the anionic nature 
of the anodic oxide formed on $\mathrm{Rh}$ in base, that oxygen evolution occurs at surface complexes that can be represented as $\left[\mathrm{Rh}(\mathrm{Iv}) \mathrm{O}_{m}(\mathrm{OH})_{n}\right]^{p-}$, where $p=2 m+n-4$. Indeed, similar considerations should apply to any oxide phase known to acquire a net negative charge in alkaline solution. Thus, the electrocatalytic activity of the various oxide films can be ascribed to the presence of complex anionic surface groups, consisting of octahedrally co-ordinated metal complexes - the surfaquo group. That said, it is important to emphasise here that the formulae for these anionic species are devices presented to rationalize the observed $E-\mathrm{pH}$ behaviour where the exact surface stoichiometry is often unknown. However, we believe that this approach is more realistic than thinking in terms of discrete stoichiometric oxy-hydroxide species.

In view of these considerations, we now outline a number of current mechanistic interpretations of the OER at oxide electrodes, taking into account the nature of the metal surfaquo groups. The following general mechanism was proposed by Lyons and Floquet ${ }^{75}$ for the OER at $\mathrm{RuO}_{2}$ and $\mathrm{IrO}_{2}$ electrodes,

$$
\begin{aligned}
& {\left[\mathrm{MO}_{m}(\mathrm{OH})_{n}\left(\mathrm{OH}_{2}\right)_{y}\right]^{p-}+\mathrm{OH}^{-} \leftrightarrow } {\left[\mathrm{MO}_{m}(\mathrm{OH})_{n}\left(\mathrm{OH}^{*}\right)\left(\mathrm{OH}_{2}\right)_{y-1}\right]^{p-} } \\
&+\mathrm{H}_{2} \mathrm{O}+\mathrm{e}^{-} \\
& {\left[\mathrm{MO}_{m}(\mathrm{OH})_{n}\left(\mathrm{OH}^{*}\right)\left(\mathrm{OH}_{2}\right)_{y-1}\right]^{p-} \rightarrow\left[\mathrm{MO}_{m}(\mathrm{OH})_{n+1}\left(\mathrm{OH}_{2}\right)_{y-1}\right]^{p-} }
\end{aligned}
$$

$$
\begin{gathered}
{\left[\mathrm{MO}_{m}(\mathrm{OH})_{n+1}\left(\mathrm{OH}_{2}\right)_{y-1}\right]^{p-} \rightarrow\left[\mathrm{MO}_{m}(\mathrm{OH})_{n+1}\left(\mathrm{OH}_{2}\right)_{y-1}\right]^{(p-1)-}+\mathrm{e}^{-}} \\
{[\mathrm{AIII})} \\
{\left[\mathrm{MO}_{m}(\mathrm{OH})_{n+1}\left(\mathrm{OH}_{2}\right)_{y-1}\right]^{(p-1)-}+2 \mathrm{OH}^{-} \leftrightarrow} \\
{\left[\mathrm{MO}_{m}(\mathrm{OH})_{n-1}\left(\mathrm{OH}_{2}\right)_{y-1}\right]^{(p-1)-}+\mathrm{O}_{2}+2 \mathrm{H}_{2} \mathrm{O}+2 \mathrm{e}^{-}}
\end{gathered}
$$

$$
\begin{aligned}
& {\left[\mathrm{MO}_{m}(\mathrm{OH})_{n-1}\left(\mathrm{OH}_{2}\right)_{y-1}\right]^{(p-1)-}+\mathrm{OH}^{-}+\mathrm{H}_{2} \mathrm{O} \leftrightarrow} \\
& \quad\left[\mathrm{MO}_{m}(\mathrm{OH})_{n}\left(\mathrm{OH}_{2}\right)_{y}\right]^{p-}
\end{aligned}
$$

where $\mathrm{M}=\mathrm{Ru}$ or Ir. In the case of $\mathrm{RuO}_{2}$ the initial oxidation state in the sequence is $\mathrm{Ru}(\mathrm{vI})$ and so $p=2 m+n-6$ whereas for $\mathrm{IrO}_{2}$ an initial oxidation state of $\operatorname{Ir}(\mathrm{Iv})$ is assumed and $p=2 m+$ $n-4$. Significantly, this mechanism was shown to be in excellent agreement with the experimental data obtained in both acid and base. By way of illustration, the key surfaquo groups and reaction steps for the OER at $\mathrm{RuO}_{2}$ and $\mathrm{IrO}_{2}$ films in base are presented in schematic form in Fig. 29.

The first step (AI) in the mechanistic sequence involves the initial discharge of a hydroxide ion at the oxide surface resulting in an increase of 1 in the metal oxidation state. The important feature here is the formation of a higher energy surface bound hydroxyl group $\mathrm{OH}^{*}$. Hence, the second step (AII) depicts a restructuring process to accommodate this energetically inequivalent hydroxyl group. While the specific details of this process are unknown, it is thought to involve a restructuring of the hydrogen bonding network surrounding the active site. Molecular dynamic simulations of metal adsorbed hydroxyl groups suggest that the hydrogen bonding surrounding a surface bound $\mathrm{OH}$ species differs from that of a surface water molecule. ${ }^{329}$ Specifically, it is shown that the oxygen-oxygen distances in the $\mathrm{OH}$ hydrogen bonded complex are shorter than for the other water molecules. This situation is similar to that observed in bulk solution, ${ }^{330,331}$ and implies that the rate-limiting step for the process of the $\mathrm{OH}$ migration, either in solution or on a surface, is the rearrangement of the local oxygen environment and not the proton transfer event. $^{329-331}$ In this way, the first two steps may be viewed analogously as an electron transfer facilitated migration of $\mathrm{OH}^{-}$ from solution onto the surface, with a fast initial discharge step and a slow hydrogen bonding restructuring process. Indeed, this purely chemical step is shown to be rate-determining for $\mathrm{IrO}_{2}$ in acid, predicting a Tafel slope of $60 \mathrm{mV} \mathrm{dec}{ }^{-1}$ and a reaction order $m_{\mathrm{OH}^{-}}=1$ or $m_{\mathrm{H}^{+}}=-1$.

The third step (AIII) is thought to involve the formation of an unstable high oxidation state Ir(VII) or Ru(VIII) surfaquo group, which serves as a precursor to di-oxygen. As illustrated in Fig. 29, this step is shown to be rate-determining for $\mathrm{IrO}_{2}$ in base and for $\mathrm{RuO}_{2}$ over the entire $\mathrm{pH}$ range with a characteristic

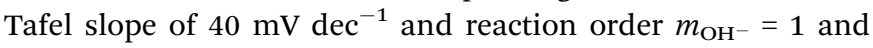
$m_{\mathrm{H}^{+}}=-1$. The fourth and final steps, involving the formation of molecular oxygen and regeneration of the catalytic centre respectively, are therefore assumed to be fast. Finally, it should be noted that at high overpotentials rate control changes to the initial discharge step for both $\mathrm{IrO}_{2}$ and $\mathrm{RuO}_{2}$ across the entire $\mathrm{pH}$ range, thereby accounting for the experimentally observed increase in Tafel slope to $c a .120 \mathrm{mV} \mathrm{dec}^{-1}$.

In a similar treatment of the OER at oxidized transition metal substrates in base, Lyons and Brandon ${ }^{14-16,92}$ proposed the following general mechanism as a feasible pathway for $\mathrm{Ni}$, Fe and Co based electrodes,

$$
\begin{gathered}
{\left[\mathrm{MO}_{m}(\mathrm{OH})_{n}\right]^{p-}+\mathrm{OH}^{-} \rightarrow\left[\mathrm{MO}_{m}(\mathrm{OH})_{n+1}\right]^{p-}+\mathrm{e}^{-}(\mathrm{BI})} \\
{\left[\mathrm{MO}_{m}(\mathrm{OH})_{n+1}\right]^{p-} \rightarrow\left[\mathrm{MO}_{m}(\mathrm{OH})_{n-1}\right]^{(p-1)-}--\mathrm{H}_{2} \mathrm{O}_{2}+\mathrm{e}^{-} \ldots \mathrm{RDS}} \\
{\left[\mathrm{MO}_{m}(\mathrm{OH})_{n-1}\right]^{(p-1)-}--\mathrm{H}_{2} \mathrm{O}_{2}+2 \mathrm{OH}^{-} \rightarrow\left[\mathrm{MO}_{m}(\mathrm{OH})_{n}\right]^{p-}} \\
--\mathrm{HO}_{2}+\mathrm{H}_{2} \mathrm{O}+\mathrm{e}^{-} \\
{\left[\mathrm{MO}_{m}(\mathrm{OH})_{n}\right]^{p-}--\mathrm{HO}_{2}+\mathrm{OH}^{-} \rightarrow\left[\mathrm{MO}_{m}(\mathrm{OH})_{n}\right]^{p-}+\mathrm{O}_{2}+\mathrm{H}_{2} \mathrm{O}+\mathrm{e}^{-}}
\end{gathered}
$$

Alternatively, the latter sequence can be represented as, ${ }^{281}$

$$
\left[\mathrm{MO}_{m}(\mathrm{OH})_{n}\right]^{p-}+\mathrm{OH}^{-} \rightarrow\left[\mathrm{MO}_{m+1}(\mathrm{OH})_{n-1}\right]^{p-}+\mathrm{H}_{2} \mathrm{O}+\mathrm{e}^{-}
$$

$$
\left[\mathrm{MO}_{m+1}(\mathrm{OH})_{n-1}\right]^{p-} \rightarrow\left[\mathrm{MO}_{m} \mathrm{OOH}(\mathrm{OH})_{n-2}\right]^{(p-1)-}+\mathrm{e}^{-} \ldots \mathrm{RDS}
$$

$$
\begin{aligned}
& {\left[\mathrm{MO}_{m} \mathrm{OOH}(\mathrm{OH})_{n-2}\right]^{(p-1)-}+2 \mathrm{OH}^{-} \rightarrow } {\left[\mathrm{MO}_{m} \mathrm{OO}(\mathrm{OH})_{n-1}\right]^{p-} } \\
&+\mathrm{H}_{2} \mathrm{O}+\mathrm{e}^{-} \\
& {\left[\mathrm{MO}_{m} \mathrm{OO}(\mathrm{OH})_{n-1}\right]^{p-}+\mathrm{OH}^{-} \rightarrow\left[\mathrm{MO}_{m}(\mathrm{OH})_{n}\right]^{p-}+\mathrm{O}_{2}+\mathrm{e}^{-} }
\end{aligned}
$$

In either case, $\mathrm{M}=\mathrm{Ni}(\mathrm{III}), \mathrm{Fe}(\mathrm{vI})$ or $\mathrm{Co}(\mathrm{IV})$ and $p=2 m+n-x$ where $x$ is the initial oxidation state of the metal centre. Taking the example of a Ni(III) centred complex $p=2 m+n-x$ and noting that $m=1$ is most likely, the catalytic $\left[\mathrm{Ni}(\mathrm{III}) \mathrm{O}_{m}(\mathrm{OH})_{n}\right]^{p-}$ 
OER Catalytic Cycle for Thermally Prepared $\mathrm{RuO}_{2}$

Electrodes in Base
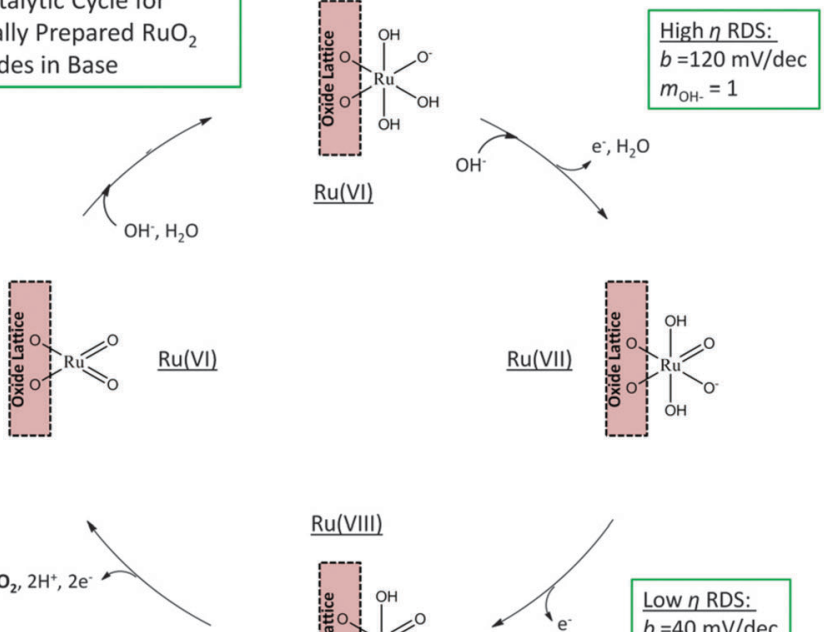

(a)

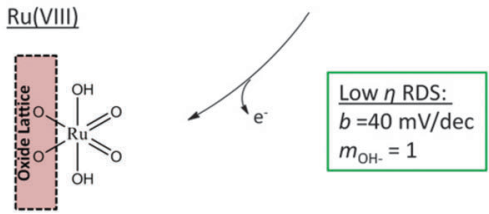

OER Catalytic Cycle for Thermally Prepared IrO Electrodes in Base $\mathrm{O}_{2}, \mathrm{H}_{2} \mathrm{O}, 2 \mathrm{e}$
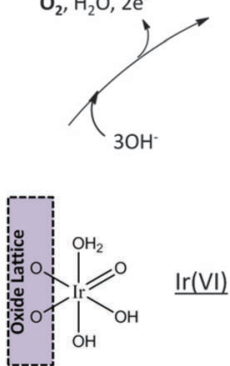

(b)

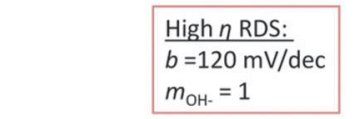<smiles>CCC(C=O)C(C)CO</smiles>

$\underline{\operatorname{lr}(\mathrm{V})}$

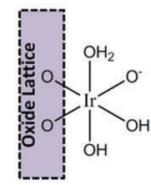

Fig. 29 Mechanism of the OER at thermally prepared (a) $\mathrm{RuO}_{2}$ and (b) IrO films in alkaline solution.

species is merely a NiOOH surface group that has coordinated extra hydroxide ions owing to the acidic nature of the oxide.

The similarities of these mechanistic pathways notwithstanding, the important distinction here lies in the interpretation of the intermediates. Pathway $\mathrm{B}$ is a modification of a mechanism proposed by Bockris and Ottagawa ${ }^{39,184}$ in which the RDS is assumed to involve the formation of a physisorbed peroxide entity $\left(-\mathrm{H}_{2} \mathrm{O}_{2}\right)$. In this way, the dioxygen entity that will subsequently be evolved as molecular oxygen is formed by the interaction of a coordinated $\mathrm{OH}$ species with an $\mathrm{OH}^{-}$ion. On the other hand, pathway $\mathrm{C}$ has been informed by the results of DFT calculations performed by Rossmeisl et al. ${ }^{270,271}$ which suggests that the RDS is the formation of a superoxy $(\mathrm{OOH})$ species by the addition of an $\mathrm{OH}^{-}$ion on top of an adsorbed oxygen atom. For an oxidised metal electrode in aqueous alkaline solution, the latter analysis indicates that the coordination of oxygen atoms by the surface metal ions will increase as the electrode potential is raised. This process is facilitated by the progressive oxidation of the metal ion to higher valance states. Here, the initial catalytic site is assumed to have the maximum coordination $(m+n)$ permitted by the valance state and the charge $(p-)$ on the surface complex. Now, if the metal is already in its highest valence state, for example a Ni(III) centre, it can only coordinate a further $\mathrm{O}$ atom by the displacement of an $\mathrm{OH}$ species. This is depicted in step CI and the surface metal cation may now be considered to be saturated with respect to coordination, given its stronger interaction (double bond character) with the $\mathrm{O}$ atom in comparison to the $\mathrm{OH}$ group (single bond character). According to the quantum chemical analysis of Rossmeisl et al., ${ }^{270,271}$ if a further $\mathrm{OH}^{-}$ion attempts to dissociate in the vicinity of the saturated cation, it is energetically more favourable for this to proceed atop one of the 
existing coordinated $\mathrm{O}$ atoms rather than for direct coordination to occur at the metal ion. This $\mathrm{OH}^{-}$may come from the surface complex as in step CII, or more generally from solution where steps CII and CIII are replaced by, ${ }^{281}$

$$
\begin{gathered}
{\left[\mathrm{MO}_{m+1}(\mathrm{OH})_{n-1}\right]^{p-}+\mathrm{OH}^{-} \rightarrow\left[\mathrm{MO}_{m} \mathrm{OOH}(\mathrm{OH})_{n-1}\right]^{p-}+\mathrm{e}^{-}} \\
\left(\mathrm{C}^{\prime} \mathrm{II}\right) \\
{\left[\mathrm{MO}_{m} \mathrm{OOH}(\mathrm{OH})_{n-1}\right]^{p-}+\mathrm{OH}^{-} \rightarrow\left[\mathrm{MO}_{m} \mathrm{OO}(\mathrm{OH})_{n-1}\right]^{p-}+\mathrm{H}_{2} \mathrm{O}+\mathrm{e}^{-}}
\end{gathered}
$$

Similar alternative steps involving a solution $\mathrm{OH}^{-}$may also be produced for pathway B. ${ }^{14-16}$ In any case, the steps outlined here involve the weakening of a metal-oxygen bond from double bond to single bond character as in CII or from single bond to physisorbed character in BII and are deemed to be rate determining. While the details of the steps subsequent to the RDS are inevitably speculative it is proposed in $\mathrm{CIII} / \mathrm{C}^{\prime} \mathrm{III}$ that a proton is transferred from the superoxy species to an $\mathrm{OH}^{-}$ion in solution. The majority of the bonding electron density is then concentrated between the two oxygen atoms with the result that the dioxygen entity is readily displaced in CIV by the adsorption of an $\mathrm{OH}^{-}$species, leading to molecular oxygen evolution and the restoration of the active site to its original condition. In this way, oxygen evolution is viewed as the mechanism by which a metal oxide surface 'copes' with the potential driven dissociation of $\mathrm{OH}^{-}$ions from solution, where the surface metal cations are already fully coordinated in their highest valance state. ${ }^{281}$

However, irrespective of the pathway admitted, the significant point here is that both of these mechanisms are capable of accounting for the entire range of kinetic parameters observed for oxidized Ni, Fe and Co substrates. A formal kinetic analysis of step II takes the same form for both pathways and it can be shown that in the limit of low Langmuir coverage of intermediates this step predicts the Tafel slope of $40 \mathrm{mV} \mathrm{dec}^{-1}$ and reaction order $m_{\mathrm{OH}^{-}}=1$ associated with the Ni electrodes. ${ }^{14,281}$ On the other hand, for 'aged' Fe and Co substrates, ${ }^{16,92,281}$ where a significant passive oxide layer has built up on the electrode surface through extended use, the characteristic Tafel

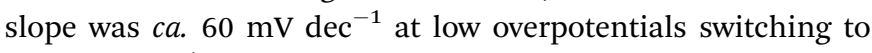
$120 \mathrm{mV} \mathrm{dec}{ }^{-1}$ at high overpotentials, with associated reaction orders $m_{\mathrm{OH}^{-}}=1.5$ and 1.0 respectively. In this case, Lyons and Brandon $^{92}$ showed that treatment of step C'II or its pathway B equivalent under Temkin adsoption conditions could account for the low overpotential kinetic data. At high overpotentials, it is assumed that the intermediate coverage has passed beyond the Temkin potential window and the kinetic data can now be interpreted under high coverage Langmuir conditions.

Furthermore, Lyons and Brandon note that the kinetic parameters associated with the 'fresher' Fe and Co electrodes, namely a Tafel slope $b \approx 2.303 \times 4 R T / 5 F$ and $m_{\mathrm{OH}^{-}}=1$, can be rationalized if a more complicated model of the electrode/ solution interface is envisaged. ${ }^{281}$ To this effect the latter authors introduced a dual barrier model developed by MacDonald and Conway ${ }^{332}$ to rationalize the OER kinetic data obtained for Au electrodes. This model, owing much to earlier work by Meyer, ${ }^{333}$ envisages that only a fraction $\Delta V_{\mathrm{FS}}$ of the

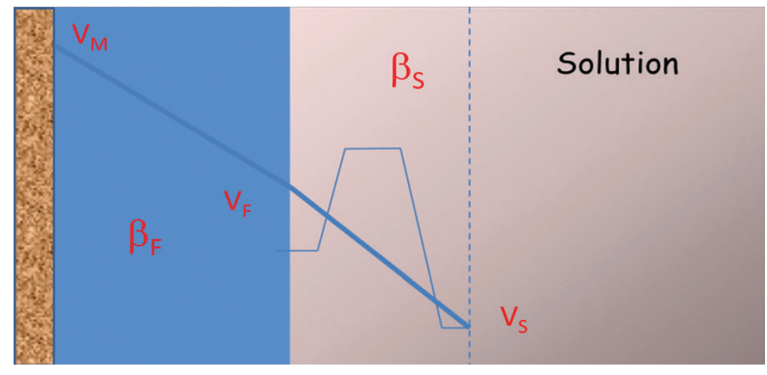

Fig. 30 Schematic representation of the Dual Barrier model for charge transfer at an oxide/solution interface.

total potential difference between the metallic electrode and the electrolyte $\Delta V_{\mathrm{MS}}=\Delta V_{\Sigma}$ is effective in lowering the potential barrier to interfacial electron transfer. In series with this, the remainder $\Delta V_{\mathrm{MF}}$ appears across an electronically conducting 'barrier' oxide, through which the charge passed in the OER must migrate under the influence of an electric field. Hence, one can visualise an inner region labeled $\mathrm{F}$ and an outer solution region labeled $S$, as shown in Fig. 30 .

Under a normal kinetic analysis of step $\mathrm{C}^{\prime} \mathrm{II}$, the overall rate equation takes the general form, ${ }^{281}$

$$
i=i_{0} \exp \left(\left(1+\beta_{\mathrm{S}}\right) F \eta / R T\right)
$$

where $i_{0}$ is the exchange current density for the OER and $\beta_{\mathrm{S}}$ denotes the symmetry factor for the interfacial electron transfer process. Now, assuming a single symmetrical barrier, $\beta_{\mathrm{S}}=1 / 2$ and the Tafel slope $b=2.303 \times 2 R T / 3 F$. On the other hand, if the OER is proceeding in the steady state under dual barrier conditions, the RDS must be in equilibrium with the barrier film charge migration process. Therefore, it is possible to obtain an expression for the overall current density across the two barriers by equating eqn (93) with the rate equation for the oxide charge migration. It can be shown that the resulting expression has the form, ${ }^{332,333}$

$$
i=A \exp \left(\left(1+\beta_{\Sigma}\right) F \eta / R T\right)
$$

where $\beta_{\Sigma}$ is the composite symmetry factor given by,

$$
\beta_{\Sigma}=\frac{\beta_{\mathrm{F}} \beta_{\mathrm{S}}}{\beta_{\mathrm{F}}+\beta_{\mathrm{S}}}
$$

In the latter expression $\beta_{\mathrm{F}}$ is the symmetry factor for field assisted charge transport through the oxide and $\beta_{\mathrm{S}}$ represents the symmetry factor for the rate determining interfacial electron transfer reaction. The pre-factor A in eqn (94) depends on $i_{0}$ and also on the exchange current density for the charge migration process, which in turn depend respectively on $a_{\mathrm{OH}^{-}}$ and the activities of the barrier film charge carriers. ${ }^{333}$ A similar dual barrier expression can be derived for the effective reaction order with respect to the activity of a particular reactant $a_{\mathrm{S}}$,

$$
\frac{\mathrm{d} \log i}{\mathrm{~d} \log a_{\mathrm{S}}}=\frac{m_{\mathrm{S}} \beta_{\mathrm{F}}}{\beta_{\mathrm{F}}+\beta_{\mathrm{S}}}
$$

where $m_{\mathrm{S}}$ is the expected value of the reaction order under more usual single interfacial electron transfer barrier conditions. 
Thus, if both potential barriers are assumed to be symmetrical $\beta_{\mathrm{F}}=\beta_{\mathrm{S}}=\frac{1}{2}$, then $\beta_{\Sigma}=\frac{1}{4}$ and a logarithmic analysis of eqn (94) gives the experimental Tafel slope $b=2.303 \times 4 R T / 5 F$ observed for oxidized Fe and Co substrates. ${ }^{281}$ In the same way, eqn (96) predicts that the reaction order $m_{\mathrm{OH}^{-}}=2$ associated with step $\mathrm{C}^{\prime}$ II or indeed its pathway B equivalent under single barrier conditions will be observed as $m_{\mathrm{OH}^{-}}=1$ under dual barrier conditions, thereby accounting for the experimentally determined reaction order for the latter electrodes. ${ }^{281}$

A final point of interest regarding the mechanistic analysis of Lyons and Brandon ${ }^{14-16,92,281}$ is their identification of the film charge transport barrier with the anhydrous inner oxide or with the interface between this region and the more dispersed hydrous outer oxide. In our discussion on the interfacial redox chemistry of Fe electrodes it was highlighted that the concept of an inner compact oxide and an outer hydrous oxide applies not only to the multicycled layers but also to the passive layer. Indeed it was shown that the hydrous oxide peak A3 can be observed from the very first potential cycle. The duplex layer model for anodic oxides developed by Burke and co-workers, ${ }^{90,91,176}$ envisages that the ions of the inner region are held in place by a rigid network of polar covalent bonds, through which ionic transport is difficult, thus limiting growth to perhaps no more than five monolayers. It is significant that this description fits the profile of a 'barrier' oxide as outlined by Meyer ${ }^{333}$ and MacDonald. ${ }^{332}$ On the other hand, charge percolation proceeds comparatively easily and quickly through the outer, hydrous, polymeric oxide region; the average rate of charge diffusion for a hydrous oxide covered Fe electrode in base is comparable to that of electrodes modified by redox polymers such as poly(pyrrole). ${ }^{17,92}$ Interestingly, a duplex model was also specifically proposed for the anodic oxide formed on $\mathrm{Au}$ electrodes $^{334}$ - the very system for which the twin barrier model was originally applied to the OER. ${ }^{332}$

The final mechanism to be discussed pertains to the hydrous oxide coated metal substrates. In this case, the latter dual barrier considerations are not expected to apply as the properties of the oxide film will be dominated by the extensive hydrous layer produced during the multicycling procedure. The following general mechanistic sequence has been proposed to rationalise the OER kinetic data observed for both $\mathrm{Fe}$ and $\mathrm{Ni}$ hydrous oxide films in base, ${ }^{96,98,218}$

$$
\begin{aligned}
\mathrm{SOH}_{2}+\mathrm{OH}^{-} & \rightarrow \mathrm{SOH}^{-}+\mathrm{H}_{2} \mathrm{O} \\
\mathrm{SOH}^{-} & \rightarrow \mathrm{SOH}+\mathrm{e}^{-} \\
\mathrm{SOH}+\mathrm{OH}^{-} & \rightarrow \mathrm{SO}^{-}+\mathrm{H}_{2} \mathrm{O} \\
\mathrm{SO}^{-} & \rightarrow \mathrm{SO}+\mathrm{e}^{-} \\
\mathrm{SO}+\mathrm{OH}^{-} & \rightarrow \mathrm{SOOH}+\mathrm{e}^{-} \\
\mathrm{SOOH}+\mathrm{OH}^{-} & \rightarrow \mathrm{SO}_{2}+\mathrm{H}_{2} \mathrm{O}+\mathrm{e}^{-} \\
\mathrm{SO}_{2}+\mathrm{OH}^{-} & \rightarrow \mathrm{SOH}^{-}+\mathrm{O}_{2}
\end{aligned}
$$

where $S$ represents a surfaquo group attached to the hydrous oxide surface by bridging oxygen ligands. A more detailed representation is presented schematically in Fig. 31 . Note that octahedrally coordinated oxymetal surfaquo groups are identified as the catalytically active species and are located within the hydrous layer. This mechanism takes advantage of the fact that the dispersed hydrous layer contains considerable quantities of water molecules which facilitate hydroxide ion discharge at the metal catalytic site. In this way our mechanistic thinking is guided by the earlier work of Kobussen and Broers, ${ }^{296,301}$ but also takes into account recent developments in DFT calculations $^{270,271,335}$ and homogeneous OER catalysis. ${ }^{323,324}$

The results of a comprehensive kinetic analysis of pathway $\mathrm{D}$ are summarised in Table 3 where the diagnostic parameters for each step, when it is assumed to be rate-determining, are presented for low and high surface coverage of intermediates. By way of an example, we now outline a sample kinetic analysis for the primary steps in pathway D. First, we note that the first

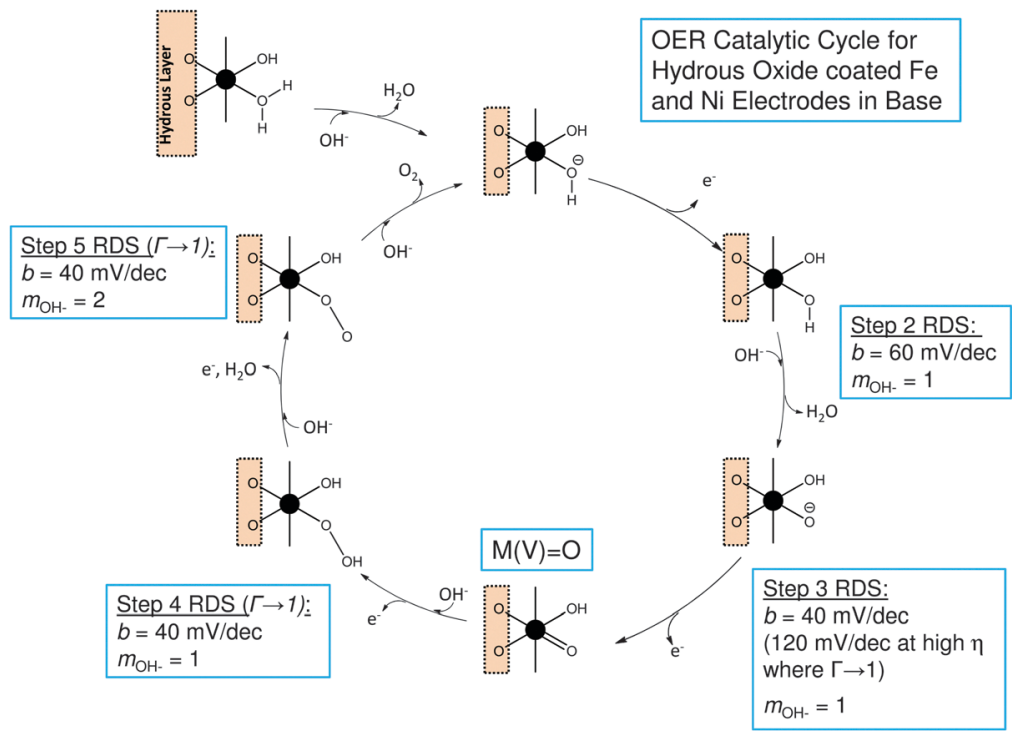

Fig. 31 Mechanism for the OER at hydrous oxide coated Fe and Ni electrodes in alkaline solution. 
Table 3 Kinetic parameters derived for mechanistic pathway D under Langmuir adsorption conditions

\begin{tabular}{llllll}
\hline & \multicolumn{1}{l}{$\Gamma \rightarrow 0$} & & & $\Gamma \rightarrow 1$ & \\
\cline { 2 - 3 } Rate-determining step & $b$ & $m_{\mathrm{OH}^{-}}$ & & $b$ & $m_{\mathrm{OH}^{-}}$ \\
\hline DII & $2 P$ & 0 & & $2 P$ & 0 \\
DIII & $P$ & 1 & & $P$ & 1 \\
DIV & $2 P / 3$ & 1 & & $2 P$ & 1 \\
DV & $2 P / 5$ & 2 & & $2 P / 3$ & 1 \\
DVI & $2 P / 7$ & 3 & & $2 P / 3$ & 2 \\
DVII & $P / 4$ & 4 & & $P$ & 2
\end{tabular}

Note: $P=2.303 R T / F$ and $\beta=\frac{1}{2}$.

step in the reaction sequence occurs rapidly and need not be included in the steady-state kinetic analysis. Therefore, if we assume that the step outlined in DIII is rate determining then the net reaction flux is given by,

$$
f_{\Sigma}=i / 4 F A=k_{2} a_{\mathrm{OH}^{-}} \Gamma_{\mathrm{SOH}}
$$

In the latter expression, $k_{2}$ is the chemical rate constant for the step outlined in DIII, $a \mathrm{OH}^{-}$represents the activity of the hydroxyl ion and $\Gamma$ denotes the surface coverage. We use the quasi steady-state approximation for the surface coverage of $\mathrm{SOH}$ to get,

$$
\frac{\mathrm{d} \Gamma_{\mathrm{SOH}}}{\mathrm{d} t}=k_{1}^{\prime} \Gamma_{\mathrm{SOH}^{-}}-k_{-1}^{\prime} \Gamma_{\mathrm{SOH}}-k_{2} \Gamma_{\mathrm{SOH}} a_{\mathrm{OH}^{-}} \cong 0
$$

where $k_{1}^{\prime}$ and $k_{-1}^{\prime}$ are the heterogeneous electrochemical rate constants for the forward and reverse reactions in DII. We may readily solve eqn (98) to obtain an expression for the surface coverage,

$$
\Gamma_{\mathrm{SOH}} \cong k_{1}^{\prime} \Gamma_{\mathrm{SOH}^{-}} /\left(k_{-1}^{\prime}+k_{2} a_{\mathrm{OH}^{-}}\right)
$$

Hence the net flux is given by,

$$
f_{\Sigma} \cong \frac{k_{2} k_{1}^{\prime} a_{\mathrm{OH}^{-}} \Gamma_{\mathrm{SOH}^{-}}}{k_{-1}^{\prime}+k_{2} a_{\mathrm{OH}^{-}}}
$$

In the latter expression the primed quantities represent heterogeneous electrochemical rate constants whose potential dependence is assumed to be given by the Butler-Volmer rate equation,

$$
k_{n}^{\prime}=k_{n}^{0} \exp \left(\frac{\beta F \eta}{R T}\right), \quad k_{-n}^{\prime}=k_{-n}^{0} \exp \left(\frac{-(1-\beta) F \eta}{R T}\right)
$$

where $\eta$ denotes the overpotential and $\beta$ is the symmetry factor. In contrast the chemical rate constant is given by its standard value and there is no potential dependence,

$$
k_{n}=k_{n}^{0}
$$

Hence the net flux is given by,

$$
f_{\Sigma} \cong \frac{k_{2}^{0} a_{\mathrm{OH}^{-}} \Gamma_{\mathrm{SOH}^{-}} k_{1}^{0} \exp [\beta F \eta / R T]}{k_{-1}^{0} \exp [-(1-\beta) F \eta / R T]+k_{2}^{0} a_{\mathrm{OH}^{-}}}
$$

Now if the step outlined in DIII is rate determining then we can safely assume that $k_{2}^{0} \ll k_{-1}^{0}$ and so eqn (103) reduces to,

$$
f_{\Sigma} \cong k_{2}^{0} \Gamma_{\mathrm{SOH}-} a_{\mathrm{OH}^{-}}\left(k_{1}^{0} / k_{-1}^{0}\right) \exp [F \eta / R T]
$$

This expression can be readily shown to predict a reaction order of unity with respect to hydroxide ion activity and a Tafel slope at $298 \mathrm{~K}$ of $c a .60 \mathrm{mV} \mathrm{dec}{ }^{-1}(b=2.303(R T / F))$.

However, if the subsequent step outlined in DIV becomes rate limiting we can write that,

$$
f_{\Sigma} \cong k_{3}^{\prime} \Gamma_{\mathrm{SO}^{-}}
$$

Again applying the quasi steady-state approximation we can show that,

$$
\frac{\mathrm{d} \Gamma_{\mathrm{SO}^{-}}}{\mathrm{d} t}=k_{2} \Gamma_{\mathrm{SOH}} a_{\mathrm{OH}^{-}}-k_{-2} a_{\mathrm{H}_{2} \mathrm{O}} \Gamma_{\mathrm{SO}^{-}}-k_{3}^{\prime} \Gamma_{\mathrm{SO}^{-}} \cong 0
$$

where $k_{-2}$ is the chemical rate constant for the reverse reaction in DIII and $k_{3}^{\prime}$ is the heterogeneous electrochemical rate constant for the reaction in DIV. Solving for the surfaquo group coverage we obtain,

$$
\Gamma_{\mathrm{SO}^{-}} \cong k_{2}^{0} \Gamma_{\mathrm{SOH}} a_{\mathrm{OH}^{-}} /\left(k_{-2}^{0} a_{\mathrm{H}_{2} \mathrm{O}}+k_{3}^{0} \exp [\beta F \eta / R T]\right)
$$

Hence the net reaction flux is given by,

$$
f_{\Sigma} \cong \frac{k_{2}^{0} k_{3}^{0} a_{\mathrm{OH}^{-}} \Gamma_{\mathrm{SOH}} \exp [\beta F \eta / R T]}{k_{-2}^{0} a_{\mathrm{H}_{2} \mathrm{O}}+k_{3}^{0} \exp [\beta F \eta / R T]}
$$

Now substituting for $\Gamma_{\text {SOH }}$ using eqn (99) we can obtain,

$$
f_{\Sigma} \cong k_{3}^{0}\left(\frac{k_{1}^{0} k_{2}^{0}}{k_{-1}^{0} k_{-2}^{0}}\right) a_{\mathrm{H}_{2} \mathrm{O}}^{-1} a_{\mathrm{OH}^{-}} \Gamma_{\mathrm{SOH}^{-}} \exp [(1+\beta) F \eta / R T]
$$

Hence, when the electrochemical oxo generation step DIV is rate limiting the flux expression presented in eqn (109) predicts that a reaction order of unity with respect to hydroxide ion activity and a Tafel slope of $c a .40 \mathrm{mV} \mathrm{dec}{ }^{-1}$ at $298 \mathrm{~K}(b=2.303(2 R T / 3 F))$ will be observed assuming that $b=\frac{1}{2}$.

Alternatively, at high overpotentials it is reasonable to assume that $\Gamma_{\mathrm{SOH}} \cong 1$ and also $k_{-2}^{0} \gg k_{3}^{0}$. Thus, eqn (108) reduces to,

$$
f_{\Sigma} \cong k_{3}^{0} a_{\mathrm{H}_{2} \mathrm{O}}^{-1}\left(k_{2}^{0} / k_{-2}^{0}\right) a_{\mathrm{OH}^{-}} \exp [\beta F \eta / R T]
$$

Hence, when the electrochemical oxo generation step DIV is rate limiting at high overpotentials the flux expression presented in eqn (110) predicts that a reaction order of unity with respect to hydroxide ion activity and a Tafel slope of $c a .120 \mathrm{mV}$ $\operatorname{dec}^{-1}$ at $298 \mathrm{~K}(b=2.303(2 R T / F))$ will be observed, again assuming that $b=\frac{1}{2}$. In this way, Lyons and co-workers ${ }^{96,98,218}$ note that this mechanism is in excellent agreement with the available kinetic data for hydrous $\mathrm{Fe}$ and $\mathrm{Ni}$ oxide films. In the limit of low surface coverage of intermediates, step DIII is rate-determining for hydrous $\mathrm{Ni}$ oxide films in low base concentrations and for Type A hydrous Fe oxide films whereas, step DIV is rate-determining for hydrous $\mathrm{Ni}$ oxide films in concentrated base solutions and for Type B Fe hydrous oxide films. On the other hand, at high overpotentials the surface coverage tends towards unity and step DIV becomes rate-determining for the whole range of hydrous oxide layers.

In view of the above kinetic analysis, we wish to highlight here a number of important features of pathway D. Firstly, it is should be noted that the proposed catalytic cycle for the OER 
outlined in Fig. 31 is analogous to those depicted for various homogeneous catalyst systems. ${ }^{316,323,324}$ This is not unexpected given the very dispersed and somewhat tenuous nature of the catalytically active hydrous oxide layer. A common feature of these reaction schemes is that the initial catalytic step involves the deprotonation of a metal coordinated water molecule. However, in the strongly alkaline conditions used in the study of the OER at hydrous oxide systems it is likely that a significant proportion of these coordinated water molecules will already be deprotonated. The $\mathrm{p} K_{\mathrm{a}}$ value for a water molecule coordinated to a highly charged metal atom is generally in the range $\mathrm{p} K_{\mathrm{a}} 5-9 .^{336}$ In light of this, Doyle and Lyons ${ }^{96,98}$ point out that it is more reasonable to assume that the initial deprotonation step is facile and will occur outside of the catalytic cycle. Hence, the initial deprotonation step is depicted as a pre-step in Fig. 31 and the OER catalytic cycle begins with the resultant coordinated $\mathrm{OH}^{-}$ ion which we label $\mathrm{SOH}^{-}$.

Secondly, it is clear that one of the key steps in pathway D involves the formation of a surface bound metal oxo SO species. In Fig. 31 this species is depicted as $\mathbf{M}=\mathrm{O}$ suggesting an $\mathrm{M}(\mathrm{v})$ metal centre. However, this species could also be represented as a metal oxyl moiety $\mathrm{M}(\mathrm{Iv})-\mathrm{O}$. In actual fact, the degree of radical character has been shown to depend on the length of the metal oxo bond with $\mathrm{M}(\mathrm{v})=\mathrm{O}$ being more stable for shorter bond lengths. ${ }^{322,323}$ In the case of $\mathrm{Fe}$, it is likely that the metal oxo species involves an $\mathrm{Fe}(\mathrm{v})$ metal centre. Indeed, a recent variable temperature mass spectrometry investigation has identified an $\mathrm{Fe}(\mathrm{v})$ oxo species as the catalytic centre in a biomimetric non-heme Fe complex. ${ }^{337}$ In the case of Ni, the situation is less clear. The Ni-O bond may be longer and have a greater radical character and a $\mathrm{Ni}(\mathrm{IV})$ oxyl intermediate may be the better descriptor of the intermediate species.

Finally, we note that the formation of metal oxide $\mathrm{SO}^{-}$(DIII), metal oxo SO (DIV) and metal peroxide SOOH (DV) species have been designated as possible rate determining steps in pathway D. To date, atomic-scale insight into the OER has proven difficult due to the lack of significant spectroscopic evidence of intermediates. However, recent surface-enhanced Raman spectroscopic (SERS) studies provide convincing evidence for $\mathrm{SOOH}$ intermediates on $\mathrm{Au}, \mathrm{Ni}$ and Co substrates. ${ }^{25,27,338}$ Specifically, Bell and coworkers ${ }^{338}$ found that a characteristic $v(\mathrm{O}-\mathrm{O})$ band of quite low intensity at $815-830 \mathrm{~cm}^{-1}$ could only be observed for a Au substrate at potentials associated with active oxygen evolution. Indeed, the low intensity of the $v(\mathrm{O}-\mathrm{O})$ band in this study highlights the difficulty in obtaining direct experimental evidence for OER intermediates. On the other hand, Muckermann and coworkers ${ }^{335}$ propose, based on DFT calculations, that for a GaN/ZnO surface with high coverage of adsorbed $\mathrm{OH}^{-}$ions the intermediate associated with the highest energy is an oxide radical. Similarly, Rossmeisl et al. ${ }^{271}$ performed DFT studies on the OER at $\mathrm{RuO}_{2}$ surfaces. They too found that for a surface saturated with adsorbed $\mathrm{OH}$, the highest energy intermediate was a surface oxygen species, in this case an oxo species. Furthermore, the latter authors assigned the highest energy state to a peroxide intermediate when the surface was saturated with adsorbed oxo rather than hydroxide species. Therefore, considering these studies, the present mechanistic interpretation brings together a number of strands in the current understanding of the OER at metal oxide electrodes, and reflects current thinking in the allied field of water oxidation in homogeneous catalytic systems via transition metal complexes.

\subsection{Electrocatalytic performance - turnover frequencies}

In recent years, the concept of a turnover frequency (TOF) has been increasingly applied to surface catalysis. This concept is well established for molecular catalysts and is defined as the number of moles of product per active site per unit time. To facilitate electrocatalytic comparisons between various surface oxide materials, current densities at a particular overpotential are often quoted or plotted as a function of some quantitative parameter associated with the electrocatalytic material. For example, the electrocatalytic activity of a hydrous Fe oxide film, as measured by the current density at an overpotential of $c a .0 .4 \mathrm{~V}$, is observed to increase as a function of the charge associated with the hydrous Fe oxide anodic peak in Fig. 32a. ${ }^{96}$ However, while the current density is indeed a measure of the rate of the reaction and therefore an indicator of electrocatalytic activity, it is not a measure of the specific electrocatalytic ability of the
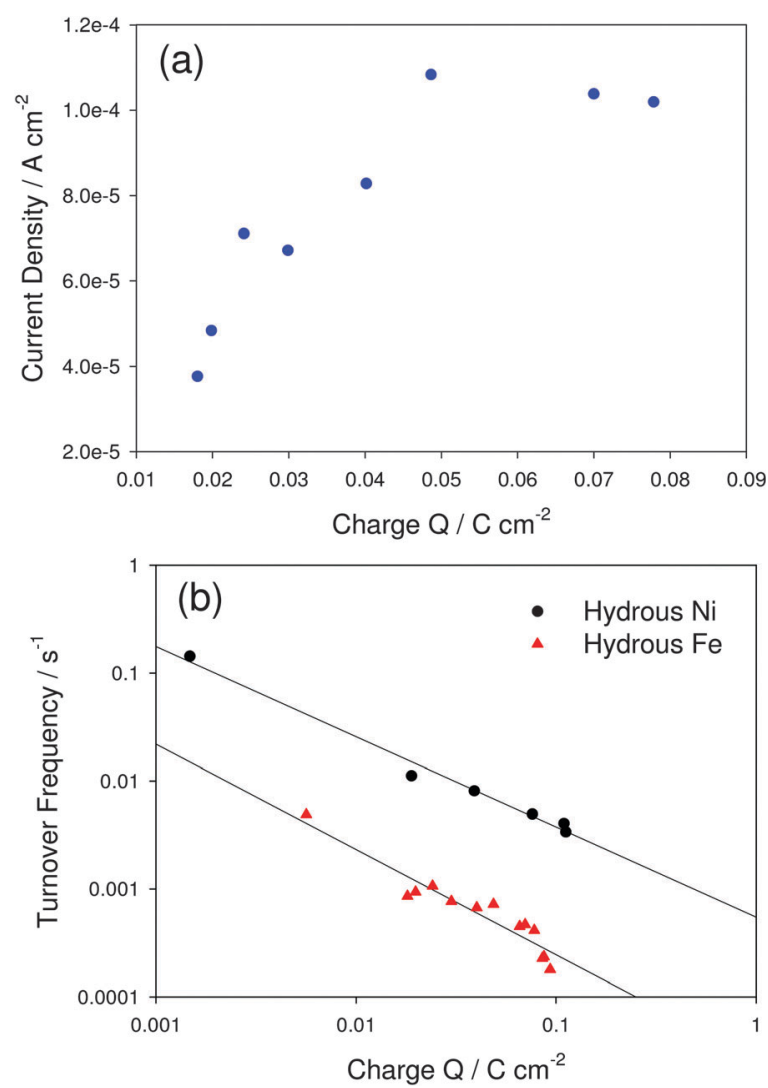

Fig. 32 (a) Variation of the current density at a fixed OER overpotential of ca. $0.4 \mathrm{~V}$ as a function of the oxide charge capacity $Q$ for a series of hydrous Fe oxide coated electrodes. (b) log-log plot of the turnover frequency calculated at the latter OER overpotential for hydrous $\mathrm{Fe}$ and $\mathrm{Ni}$ oxide coated electrodes as a function of $Q$. 
system under scope. ${ }^{339}$ This is due to the fact that the current density is expected to be proportional to the amount of catalyst present, as was observed for the hydrous Fe oxide film in Fig. 32a. In this respect, the specific electrocatalytic ability of the system can be given in terms of the TOF, which for the OER may be defined as the number of oxygen molecules generated per surface catalytic site per second. Accordingly, the TOF can be calculated as follows, ${ }^{27,339}$

$$
\mathrm{TOF}=\frac{1}{N_{\text {cat }}} \frac{\mathrm{d} N_{\mathrm{O}_{2}}}{\mathrm{~d} t}=\frac{i_{E} N_{\mathrm{A}}}{n F N_{\text {cat }}}=\frac{1}{4} \frac{j_{E}}{Q}
$$

where $N_{\text {cat }}$ is the number of catalytic sites, $N_{\mathrm{O}_{2}}$ is the number of molecules of oxygen produced, $N_{\mathrm{A}}$ is Avogadro's number, $Q$ is the charge and, $i_{E}$ and $j_{E}$ are the current and current density at a potential $E$ associated with oxygen evolution.

In Fig. $32 \mathrm{~b}$ the calculated TOF values for a series of hydrous $\mathrm{Fe}$ and $\mathrm{Ni}$ oxide electrodes are plotted, in double logarithmic form, as a function of $Q$. In contrast to the trend observed in Fig. 32a, the TOF decreases with increasing oxide charge capacity $Q$. That is, the specific electrocatalytic performance decreases with increasing quantities of catalyst. Similar observations have also been made by Gomez et al. ${ }^{339}$ for a series of nanostructured $\mathrm{Ni}(\mathrm{OH})_{2}$ layers. The reasons behind this behaviour are unclear. There may be an inherent overestimation in the number of electrocatalytic sites. The obvious assumption being made here is that all the electroactive metal atoms giving rise to $Q$ also act as catalytic sites, which may or may not be reasonable depending on the structure and morphology of the surface oxide. Indeed, Gomez et al. ${ }^{339}$ note that the apparent fraction of metal atoms exposed to the electrolyte will likely decrease with increasing quantities of deposited catalyst, thereby decreasing the relative electrocatalytic surface. Alternatively, it has been suggested that charge transport may be a limiting factor for thicker deposits. ${ }^{339}$ For very thin layers electron transfer to the conducting substrate would be facile. However, with increasing thickness, an increased fraction of the layer would be dependent on charge transport processes through the layer for its electrocatalytic functioning. In any case, it is evident from Fig. 32b that the TOF offers a simple comparison of electrocatalytic performance for surface materials, clearly identifying the $\mathrm{Ni}$ based electrodes as the more catalytically active material.

The determination of TOFs carries further significance in that it facilitates a comparison between heterogeneous surface catalysis and homogeneous molecular catalysis. The reported TOF values for a selection of surface and solution based systems are presented in Table 4. Although a wide range of TOFs are possible for both types of system an obvious trend emerges from their comparison; the TOF for a solution based catalyst is typically greater than for its surface based equivalent. This trend is seemingly independent of catalytic ability and can be clearly noted in Table 4 from the $\mathrm{Co}_{3} \mathrm{O}_{4}$ and colloidal $\mathrm{IrO}_{2}$ data. This reduction in catalytic activity for surface immobilised systems is not unexpected. In solution the catalyst represents a possible 3-dimensional and highly mobile active site, whereas surface sites will be significantly more restricted both
Table 4 OER turnover frequencies for various surface and solution based catalyst systems

\begin{tabular}{|c|c|c|}
\hline \multirow[b]{2}{*}{ Material } & \multicolumn{2}{|l|}{$\mathrm{TOF} / \mathrm{s}^{-1}$} \\
\hline & Surface & Solution \\
\hline Photosystem $\mathrm{II}^{340,341}$ & 100-400 (in vivo) & ca. 1000 (in vitro) \\
\hline$\left[\mathrm{Ru}(\mathrm{bda})(\mathrm{isoq})_{2}\right]^{316}$ & - & ca. 300 \\
\hline$\left[\mathrm{Ru}_{2}(\mu-\mathrm{OAc})(\mathrm{bpp})(\mathrm{tpy})_{2}\right]^{2+320}$ & - & 0.014 \\
\hline $\mathrm{Co}_{3} \mathrm{O}_{4}{ }^{342-344}$ & $0.0008-0.02$ & $0.035-0.055$ \\
\hline Colloidal $\mathrm{IrO}_{2}^{342,345-348}$ & 7 & 40 \\
\hline Thermally prepared $\mathrm{IrO}_{2}{ }^{a}$ & 0.003 & - \\
\hline Thermally prepared $\mathrm{RuO}_{2}{ }^{a}$ & 0.014 & - \\
\hline Aged $\beta$-Ni $(\mathrm{OH})_{2}{ }^{a}$ & 0.95 & - \\
\hline Hydrous Ni oxide ${ }^{a}$ & 0.003 & - \\
\hline Hydrous Fe oxide ${ }^{a}$ & 0.001 & - \\
\hline $\mathrm{MnO}_{2}{ }^{342,349}$ & 0.013 & - \\
\hline $\mathrm{Mn}_{2} \mathrm{O}_{3}^{342,344}$ & - & $0.035-0.055$ \\
\hline Nafion modified $\left[\mathrm{Mn}_{4} \mathrm{O}_{4} \mathrm{~L}_{6}\right]^{+}$ & - & 0.075 \\
\hline
\end{tabular}

sterically and in terms of their mobility. Indeed, even the oxygen evolving complex of photosystem II experiences up to a ten-fold reduction in catalytic activity in vivo as opposed to in vitro. ${ }^{340,341}$ In addition, Bard et al. ${ }^{350}$ have noted that the heterogeneous electrochemical rate constants for reactants bound in a surface layer are generally 2-3 orders of magnitude smaller than those for the same reactants in the solution phase. In light of this, a direct comparison between surface based and solution based catalysts seems unreasonable. However, one must ask the question: What is the minimum TOF required of a surface layer for its catalytic performance to approach that of photosystem II?

\section{Conclusion}

We have reviewed the preparation, redox switching behaviour and electrocatlytic properties of three classes of transition metal oxide electrodes: DSA type electrodes, hydrous oxide electrodes and bulk oxide/hydroxide electrodes. The characteristic structural and physical properties of the metal oxides are highly influenced by the manner in which they are prepared. Key factors include: thermal annealing temperature, electrochemical scanning frequency and potential, electrode substrate, and deposition solution. In view of the wide variety of possible oxide materials, we have sought to highlight in this review some unifying concepts pertaining to their redox and electrocatalytic properties.

The redox behaviour of transition metal oxide electrodes has been interpreted in terms of anionic surface bound octahedrally coordinated metal complexes known as surfaquo groups. This interpretation arises from the observed acid/base properties of the oxide materials. While the spectroscopic determination of the exact composition and structure of these anionic entities is rendered experimentally challenging by issues such as sample preparation, transfer from solution to vacuum and issues regarding the meaning/reliability of data acquired $e x$ situ; the characteristic $E-\mathrm{pH}$ response of the oxide provides a useful insight into the nature of the surface oxide. 
Following this interpretation, redox switching of the oxide layer involves electron transfer along connected surfaquo groups located not only at the oxide/solution interface, as in the DSA electrodes, but possibly throughout the layer for the bulk and microdispersed hydrous electrodes. The latter process is facilitated by the accompanied diffusion of protons or hydroxide ions and can be readily studied both qualitatively and quantitatively using simple potential sweep techniques; diffusion coefficients in the range of $10^{-8}$ to $10^{-10} \mathrm{~cm}^{2} \mathrm{~s}^{-1}$ reported for the hydrous materials indicate charge transport rates comparable to other electroactive polymers.

The electrocatalytic properties of transition metal oxides have been described in relation to the industrially and commercially significant oxygen evolution reaction. We have focussed here on a classical electrochemical approach, outlining the practical and theoretical application of steady-state polarisation curves, open circuit potential decay curves and electrochemical impedance spectroscopy. In particular, the benefits and importance of utilising an ensemble of electrochemical techniques for the elucidation of mechanistically significant kinetic parameters such as Tafel slopes and reaction orders has been emphasised. The latter approach was shown to provide a detailed kinetic analysis of the OER enabling one to identify and distinguish between possible mechanistic pathways.

In this respect we have provided an overview of some classical and modern trends in the interpretation of the OER. Specifically, we note the move from a more macroscopic viewpoint in terms of the surface properties of the oxide to a more microscopic viewpoint in terms of the atomic/molecular scale properties of the layer. Certainly, knowledge of the chemical and structural properties of the underlying oxide phase is important in understanding the OER; the amphoteric character of the anodic oxides implies that it is more realistic to view the OER active sites in terms of anionic surface complexes rather than the traditional viewpoint of stoichiometric units of the bulk oxide material. Subsequently, a series of novel OER mechanistic pathways involving the active participation of metal oxyhydroxide surfaquo groups have been discussed. These mechanistic interpretations have been inspired by the classic works of Kobussen, ${ }^{296,301}$ Bockris $^{39,184}$ and O'Grady, ${ }^{300}$ while also resonating with more recent spectroscopic ${ }^{25,27,338}$ and DFT studies, ${ }^{270,271,335}$ and current work concerning various oxygen evolving molecular inorganic transition metal complexes. ${ }^{29,44}$ Importantly, the structure of the surfaquo groups mirrors that of homogeneous molecular catalysts. Taking this into consideration, the evaluation of turnover frequencies for the surface immobilised catalysts represents a potential comparative bridge with the solution based molecular catalysts, although it is clear that a certain 'proportionality' in catalytic performance needs to be accounted for. However, there seems to be a definite emerging opinion, shared by ourselves and others, ${ }^{10}$ that the key to future developments in the study of the OER relies on the integration of the allied fields of heterogeneous surface and homogeneous molecular catalysis. Indeed, we can conclude that the chemistry of the surfaquo group determines the chemistry of the OER catalytic cycle.

\section{Acknowledgements}

This publication has emanated in part from research conducted with the financial support of Science Foundation Ireland (SFI) under grant number SFI/10/IN.1/I2969. We also wish to thank the Advanced Microscopy Laboratory (AML) in the Centre for Research on Adaptive Nanostructures and Nanodevices (CRANN) at Trinity College Dublin for their help with the SEM analysis.

\section{Notes and References}

1 L. Zhou, Renewable Sustainable Energy Rev., 2005, 9, 395.

2 G. P. Dinga, J. Chem. Educ., 1985, 65, 688.

3 R. Schlogl, ChemSusChem, 2010, 3, 209.

4 G. W. Crabtree, M. S. Dresselhaus and M. V. Buchanan, Phys. Today, 2004, 57, 39.

5 P. Häussinger, R. Lohmüller and A. M. Watson, Ullmann's Encyclopedia of Industrial Chemistry, Wiley-VCH Verlag $\mathrm{GmbH} \& \mathrm{Co} . \mathrm{KGaA}, 2000$.

6 K. Zeng and D. Zhang, Prog. Energy Combust. Sci., 2010, 36, 307.

7 H. Tributsch, Int. J. Hydrogen Energy, 2008, 33, 5911.

8 J. Ohi, J. Mater. Res., 2005, 20, 3180.

9 D. E. Hall, J. Electrochem. Soc., 1983, 130, 317.

10 H. Dau, C. Limberg, T. Reier, M. Risch, S. Roggan and P. Strasser, ChemCatChem, 2010, 2, 724.

11 S. Marinia, P. Salvi, P. Nelli, R. Pesentia, M. Villa, M. Berrettoni, G. Zangaric and Y. Kiros, Electrochim. Acta, 2012, 82, 384 .

12 A. Michas, F. Andolfatto, M. E. G. Lyons and R. Durand, Key Eng. Mater., 1992, 72-74, 535.

13 K. Kinoshita, Electrochemical Oxygen Technology, Wiley, New York, 1992.

14 M. E. G. Lyons and M. P. Brandon, Int. J. Electrochem. Sci., 2008, 3, 1386.

15 M. E. G. Lyons and M. P. Brandon, Int. J. Electrochem. Sci., 2008, 3, 1425.

16 M. E. G. Lyons and M. P. Brandon, Int. J. Electrochem. Sci., 2008, 3, 1463.

17 M. E. G. Lyons, R. L. Doyle and M. P. Brandon, Phys. Chem. Chem. Phys., 2011, 13, 21530.

18 M. E. G. Lyons, L. Russell, M. O’Brien, R. L. Doyle, I. Godwin and M. P. Brandon, Int. J. Electrochem. Sci., 2012, 7, 2710.

19 T. Kessler, J. R. Vilche, M. Ebert, K. Jüttner and W. J. Lorenz, Chem. Eng. Technol., 1991, 14, 263.

20 K. K. Lian, D. W. Kirk and S. J. Thorpe, J. Electrochem. Soc., 1995, 142, 3704.

21 A. M. Fundo and L. M. Abrantes, Russ. J. Electrochem., 2006, 42, 1291.

22 S. I. Cordorba, R. B. Carbonio, M. Lopez Teijelo and V. A. Macagno, Electrochim. Acta, 1987, 32, 749.

23 Y. Zhang, X. Cao, H. Yuan, W. Zhang and Z. Zhou, Int. J. Hydrogen Energy, 1999, 24, 529.

24 X. Wang, H. Luo, D. R. Zhou, H. Yang, P. J. Sebastian and S. A. Gamboa, Int. J. Hydrogen Energy, 2004, 29, 967. 
25 B. S. Yeo and A. T. Bell, J. Phys. Chem. C, 2012, 116, 8394. 26 M. W. Kanan and D. G. Nocera, Science, 2008, 321, 1072.

27 B. S. Yeo and A. T. Bell, J. Am. Chem. Soc., 2011, 133, 5587.

28 E. B. Castro, C. A. Gervasi and J. R. Vilche, J. Appl. Electrochem., 1998, 28, 835.

29 M. S. El-Deab, M. I. Awad, A. M. Mohammad and T. Ohsaka, Electrochem. Commun., 2007, 9, 2082.

30 C. R. Davidson, G. Kissel and S. Srinivasan, J. Electroanal. Chem., 1982, 132, 129.

31 S. K. Tiwari, S. Samuel, R. N. Singh, G. Poillerat, J. F. Koenig and P. Chartiers, Int. J. Hydrogen Energy, 1995, 20, 9.

32 C. Bocca, A. Barbucci, M. Delucchi and G. Cerisola, Int. J. Hydrogen Energy, 1999, 24, 21.

33 E. Guerrini, M. Piozzini, A. Castelli, A. Colombo and S. Trasatti, J. Solid State Electrochem., 2008, 12, 363.

34 C. Iwakura, A. Honji and H. Tamura, Electrochim. Acta, 1981, 26, 1319.

35 P. Rasiyah and A. C. C. Tseung, J. Electrochem. Soc., 1983, 130, 365.

36 S. Palmas, F. Ferrara, A. Vacca, M. Mascia and A. M. Polcaro, Electrochim. Acta, 2007, 53, 400.

37 J. P Singh, N. K. Singh and R. N. Singh, Int. J. Hydrogen Energy, 1999, 24, 433.

38 R. N. Singh, J. P. Singh, B. Lal, M. J. K. Thomas and S. Bera, Electrochim. Acta, 2006, 51, 5515.

39 J. O’M. Bockris and T. Otagawa, J. Phys. Chem., 1983, 87, 2960.

40 Y. Matsumoto, S. Yamada, T. Hishida and E. Sato, J. Electrochem. Soc., 1980, 127, 2360.

41 C. Bocca, G. Cerisola, E. Magnone and A. Barbucci, Int. J. Hydrogen Energy, 1999, 24, 699.

42 R. N. Singh and B. Lal, Int. J. Hydrogen Energy, 2002, 27, 45.

43 B. Klahr, S. Gimenez, F. Fabregat-Santiago, T. Hamann and J. Bisquert, J. Am. Chem. Soc., 2012, 134, 4294.

44 B. E. Conway, Prog. Surf. Sci., 1995, 49, 331.

45 L. D. Burke and M. E. G. Lyons, in Modem Aspects of Electrochemistry, ed. J. O'M. Bockris, R. E. White and B. E. Conway, Plenum Publ. Corp., New York, vol 18, ch. 4, 1986.

46 S. Rebouillat, M. E. G. Lyons, M. P. Brandon and R. L. Doyle, Int. J. Electrochem. Sci., 2011, 6, 5830.

47 A. Daghetti, G. Lodi and S. Trasatti, Mater. Chem. Phys., 1983, 8, 1 .

48 S. Trasatti, Electrochim. Acta, 1991, 36, 225.

49 H. B. Beer, J. Electrochem. Soc., 1980, 127, 303C.

50 P. P. L. Massot, A. Savall and P. Taxil, J. New Mater. Electrochem. Syst., 2007, 10, 123.

51 G. P. Vercesi and C. H. Comninellis, J. Appl. Electrochem., 1991, 21, 335.

52 S. Trasatti, Electrochim. Acta, 2000, 45, 2377.

53 S. Trasatti, Port. Electrochim. Acta, 2001, 19, 197.

54 V. De Nora, Proc. Symp. Diaphragm Cells Chlorine Prod., 1977 , p. 15.

55 O. J. Murphy, L. D. Burke, J. O’Neill and S. Venkatesan, J. Chem. Soc., Faraday Trans., 1977, 73, 1659.
56 F. Tantardini, S. Trasatti and D. Galizzioli, J. Appl. Electrochem., 1974, 4, 47.

57 M. E. G. Lyons and L. D. Burke, J. Chem. Soc., Faraday Trans. 1, 1987, 83, 299.

58 J. P. Zheng, P. J. Cygan and T. R. Jow, J. Electrochem. Soc., 1995, 142, 2699.

59 F. I. Mattos-Costa, P. de Lima-Neto, S. A. S. Machado and L. A. Avaca, Electrochim. Acta, 1998, 44, 1515.

60 A. J. Terezo and E. C. Pereira, Mater. Lett., 2002, 53, 339.

61 H. M. Villullas, F. I. Mattos-Costa and L. O. S. Bulhoes, J. Electroanal. Chem., 2003, 545, 89.

62 D. Galizzioli, F. Tantardini and S. Trasatti, J. Appl. Electrochem., 1974, 4, 57.

63 D. Gallizioli, F. Tantardini and S. Trasatti, J. Appl. Electrochem., 1975, 5, 203.

64 G. Lodi, E. Sivieri, A. De Battisti and S. Trasatti, J. Appl. Electrochem., 1978, 8, 135.

65 Y. E. Roginskaya, O. V. Morozova, E. N. Lubnin, Y. E. Ulitina, G. V. Lopukhova and S. Trasatti, Langmuir, 1997, 13, 4621.

66 J. Haenen, W. Visscher and E. Barendrecht, J. Electroanal. Chem., 1986, 208, 273.

67 K. Koumoto and H. Yanagida, Jpn. J. Appl. Phys., 1981, 20, 445.

68 G. Lodi, C. Bighi and C. de Asmundis, Mater. Sci., 1976, $1,177$.

69 S. Trasatti and G. Lodi, in Electrodes of Conductive Metallic Oxides, ed. S. Trasatti, Part B, Elsevier, Amsterdam, 1981, ch. 10 , p. 521.

70 V. V. Shalaginov, I. D. Belova, Y. E. Roginskaya and D. M. Shub, Elektrokhimiya, 1978, 14, 1708.

71 I. D. Belova, V. V. Shalaginov, B. Sh. Galyamov, Y. E. Roginskaya and D. M. Shub, Zh. Neorg. Khim., 1978, 23, 286.

72 C. Iwakura, K. Hirao and H. Tamura, Electrochim. Acta, 1977, 22, 335.

73 M. Morita, C. Iwakura and H. Tamura, Electrochim. Acta, 1979, 24, 357.

74 A. Carugati, G. Lodi and S. Trasatti, Mater. Chem., 1981, 6, 255.

75 M. E. G. Lyons and S. Floquet, Phys. Chem. Chem. Phys., 2011, 13, 5314.

76 R. J. Hunter, Foundations of Colloid Science, Oxford University Press, 2nd edn, 2005, Ch. 7 \& 8, p. 305.

77 S. D. James, J. Electrochem. Soc., 1969, 116, 1681.

78 J. Balej and O. Spalek, Collect. Czech. Chem. Commun., 1972, 37, 499.

79 S. Shibita, J. Electroanal. Chem., 1978, 89, 37.

80 L. D. Burke and M. McRann, J. Electroanal. Chem., 1981, $125,387$.

81 D. N. Buckley and L. D. Burke, J. Chem. Soc., Faraday Trans. 1, 1975, 71, 1447.

82 D. N. Buckley, L. D. Burke and J. K. Mulcahy, J. Chem. Soc., Faraday Trans. 1, 1976, 72, 1896.

83 D. F. Pickett and J. T. Malloy, J. Electrochem. Soc., 1978, 125, 1026. 
84 L. D. Burke and M. E. G. Lyons, J. Electroanal. Chem., 1986, 198, 347.

85 L. D. Burke and E. J. M. O'Sullivan, J. Electroanal. Chem., 1978, 93, 11.

86 L. D. Burke and D. P. Whelan, J. Electroanal. Chem., 1980, 109, 385.

87 A. C. Chialvo, W. E. Triaca and A. J. Arvia, J. Electroanal. Chem., 1983, 146, 93.

88 W. M. Sachtler and L. L. V. Reizen, J. Res. Inst. Catal., 1962, 10, 87.

89 R. Woods, in Electroanalytical Chemistry, ed. A. J. Bard, Marcel Dekker, New York, vol. 9, ch. 1, 1976.

90 L. D. Burke and E. J. M. O'Sullivan, J. Electroanal. Chem., 1981, 117, 155.

91 L. D. Burke, M. I. Casey, V. J. Cunnane, O. J. Murphy and T. A. M. Twomey, J. Electroanal. Chem., 1985, 189, 353.

92 M. E. G. Lyons and M. P. Brandon, Phys. Chem. Chem. Phys., 2009, 11, 2203.

93 P. G. Pickup and V. I. Birss, J. Electroanal. Chem., 1987, 220, 83.

94 L. D. Burke and M. B. C. Roche, J. Electroanal. Chem., 1984, 164, 315.

95 L. D. Burke, M. M. McCarthy and M. B. C. Roche, J. Electroanal. Chem., 1984, 167, 291.

96 R. L. Doyle and M. E. G. Lyons, J. Electrochem. Soc., 2013, 160, H142.

97 L. D. Burke and J. K. Mulcahy, J. Electroanal. Chem., 1976, 73, 207.

98 R. L. Doyle and M. E. G. Lyons, Phys. Chem. Chem. Phys, 2013, 15, 5224.

99 M. E. G. Lyons and R. L. Doyle, Int. J. Electrochem. Sci., 2012, 7, 9488.

100 V. Srinivasan and J. W. Weidner, J. Electrochem. Soc., 1997, 144, L210.

101 K.-C. Liu and M. A. Anderson, J. Electrochem. Soc., 1996, 143,124

102 K.-W. Nam and K.-B. Kim, J. Electrochem. Soc., 2002, 149, A346.

103 V. Srinivasan and J. W. Weidner, J. Electrochem. Soc., 2000, 147, 880 .

104 A. A. F. Grupioni and T. A. F. Lassali, J. Electrochem. Soc., 2001, 148, A1015.

105 K. W. Nam, W. S. Yoon and K. B. Kim, Electrochim. Acta, 2002, 47, 3201.

106 E. E. Kalu, T. T. Nwoga, V. Srinivasan and J. W. Weidner, J. Power Sources, 2001, 92, 163.

107 G. Fu, Z. Hu, L. Xie, X. Jin, Y. Xie, Y. Wang, Z. Zhang, Y. Yang and H. Wu, Int. J. Electrochem. Sci., 2009, 4, 1052.

108 D. Kong, J. Wang, H. Shao, J. Zhang and C. Cao, J. Alloys Compd., 2011, 509, 5611.

109 V. Srinivasan and J. W. Weidner, J. Power Sources, 2002, 108, 15.

110 H. K. Kim, T. Y. Seong, J. H. Lim, W. L. Cho and Y. S. Yoon, J. Power Sources, 2001, 102, 167.

111 C. Lin, J. A. Ritter and B. N. Popov, J. Electrochem. Soc., 1998, 145, 4097.
112 S. C. Pang, M. A. Anderson and T. W. Chapman, J. Electrochem. Soc., 2000, 147, 444.

113 S. C. Pang and M. A. Anderson, J. Mater. Res., 2000, 15, 2096.

114 R. N. Reddy and R. G. Reddy, J. Power Sources, 2003, 124, 330.

115 M. Wu, G. A. Snook, G. Z. Chen and D. J. Fray, Electrochem. Commun., 2004, 6, 499.

116 S.-F. Chin, S.-C. Pang and M. A. Anderson, J. Electrochem. Soc., 2002, 149, A379.

117 M. Toupin, T. Brousse and D. Belanger, Chem. Mater., 2002, 14, 3946.

118 B. Djurfors, J. N. Broughton, M. J. Brett and D. G. Ivey, Acta Mater., 2005, 53, 957.

119 M. Wu, L. Zhang, J. Gao, C. Xiao, D. Wang, A. Chen and S. Zhang, J. Electroanal. Chem., 2008, 613, 125.

120 J. Wei, M. Cheong, N. Nagarajan and I. Zhitomirsky, ECS Trans., 2007, 3, 1.

121 F. Xiao and Y. Xu, Int. J. Electrochem. Sci., 2012, 7, 7440.

122 L.-R. Shiue, N.-L. Wu, D.-S. Wu, C.-W. Chao and Y.-P. Lan, US Pat. No, US, 6,678,147, B2, 2004.

123 S.-Y. Wang and N.-L. Wu, J. Appl. Electrochem., 2003, 33, 345.

124 N.-L. Wu, S.-Y. Wang, C.-Y. Han, D.-S. Wu and L.-R. Shiue, J. Power Sources, 2003, 113, 173.

125 T. Brousse and D. Be'langer, Electrochem. Solid-State Lett., 2003, 6, A244.

126 N. Nagarajan and I. Zhitomirsky, J. Appl. Electrochem., 2006, 36, 1399.

127 G. W. Briggs, E. Jones and W. F. K. Wynne-Jones, Trans. Faraday Soc., 1955, 51, 1433.

128 E. Jones and W. F. K. Wynne-Jones, Trans. Faraday Soc., 1956, 52, 1260.

129 R. Barnard, C. F. Randell and F. L. Tye, J. Appl. Electrochem., 1980, 10, 109.

130 Handbook of batteries, ed. D. Linden, Mc Graw Hill, New York, 1995.

131 P. H. L. Notten, NATO ASI Ser., Ser. E, 1995, 281, 151.

132 P. H. L. Notten and J. R. G. Van Beek, Chem. Ind., 2000, 54,102 .

133 S. Trasatti, Electrochim. Acta, 1984, 29, 1503.

134 A. C. C. Tseung and S. Jasem, Electrochim. Acta, 1977, 22, 31.

135 R. S. Schrebler Guzman, J. R. Vilche and A. J. Arvia, Corros. Sci., 1978, 18, 765.

136 P. W. T. Lu and S. Srinivasan, J. Appl. Electrochem., 1979, 9, 269.

137 P. W. T. Lu and S. Srinivasan, J. Electrochem. Soc., 1978, 125, 1416.

138 D. E. Hall, J. Electrochem. Soc., 1981, 128, 740.

139 H. Willerns, A. G. C. Kobussen, J. H. W. De Witt and G. H. J. Broers, J. Electroanal. Chem., 1984, 177, 227.

140 L. Brossard, Int. J. Hydrogen Energy, 1991, 16, 87.

141 M. Cappadonia, J. Divisek, T. von der Heyden and U. Stimming, Electrochim. Acta, 1994, 39, 1559.

142 E. B. Castro and C. A. Gervasi, Int. J. Hydrogen Energy, 2000, 25, 1163. 
143 M. Risch, V. Khare, I. Zaharieva, L. Gerencser, P. Chernev and H. Dau, J. Am. Chem. Soc., 2009, 131, 6936.

144 C. K. Lin, K. H. Chuang, C. Y. Lin, C. Y. Tsay and C. Y. Chen, Surf. Coat. Technol., 2007, 202, 1272.

145 E. Ozkan Zayim, I. Turhan, F. Z. Tepehan and N. Ozer, Sol. Energy Mater. Sol. Cells, 2008, 92, 164.

146 Y. G. Wang and Y. Y. Xia, Electrochim. Acta, 2006, 51, 3223. 147 V. Subramanian, H. W. Zhu, R. Vajtai, P. M. Ajayan and B. Q. Wei, J. Phys. Chem. B, 2005, 109, 20207.

148 M. E. Folquer, J. R. Vilche and A. J. Arvia, J. Electrochem. Soc., 1980, 127, 2634.

149 K. C. Liu and M. A. Anderson, J. Electrochem. Soc., 1996, 143, 124.

150 I. Porqueras and E. Bertran, Thin Solid Films, 2001, 398/ 399, 41.

151 T. Abe, A. Taguchi, S. Inoue, S. Akamaru, M. Hara and K. Watanabe, J. Alloys Compd., 2006, 414, 137.

152 H. Y. Lee and J. B. Goodenough, J. Solid state Chem., 1999, 144, 220.

153 Y. Dai, K. Wang, J. Zhao and J. Xie, J. Power Sources, 2006, $161,737$.

154 Y. G. Wu, G. M. Wu, X. Y. Ni and X. Wu, Sol. Energy Mater. Sol. Cells, 2000, 63, 217.

155 S. H. Lee, C. E. Tracey and J. R. Pitts, Electrochem. SolidState Lett., 2004, 7, A299.

156 B. Subramanian, M. Mohamed Ibrahim, V. Senthikumar, K. R. Murali, V. S. Vidhya, C. Sanjeerviraja and M. Jayachandran, Physica B, 2008, 403, 4104.

157 A. D. Kuypers, C. I. M. A. Spee, J. L. Linden, G. Kirchner, J. F. Forsyth and A. Mackor, Surf. Coat. Technol., 1995, 74/ 75, 1033.

158 B. Djurfors, J. N. Broughton, M. J. Brett and D. G. Ivey, J. Power Sources, 2006, 156, 741.

159 N. Cherchour, C. Deslouis, B. Messaoudi and A. Pailleret, Electrochim. Acta, 2011, 56, 9746.

160 T. Subbaiah, S. C. Mallick, K. G. Mishra, K. Sanjay and R. P. Das, J. Power Sources, 2002, 112, 562.

161 M. Murthy, G. S. Nagarajan, J. W. Weidner and J. W. Van Zee, J. Electrochem. Soc., 1996, 143, 2319.

162 C. C. Streinz, A. P. Hartman, S. Motupally and J. W. Weidner, J. Electrochem. Soc., 1995, 142, 1084.

163 C. C. Streinz, S. Motupally and J. W. Weidner, J. Electrochem. Soc., 1995, 142, 4051.

164 T. Yousefi, A. N. Golikand, M. H. Mashhadizadeh and M. Aghazadeh, J. Taiwan Inst. Chem. Eng., 2012, 43, 614.

165 H. Liu, G. Yan, F. Liu, Y. Zhong and B. Feng, J. Alloys Compd., 2009, 481, 385.

166 M. Fantini and A. Gorenstein, Sol. Energy Mater., 1987, 16, 487.

167 M. E. G. Lyons, A. Cakara, P. O’Brien, I. Godwin and R. L. Doyle, Int. J. Electrochem. Sci., 2012, 7, 11768.

168 G. M. Jacob and I. Zhitomirsky, Appl. Surf. Sci., 2008, 254, 6671.

169 H. Adelkhani and M. Ghaemi, J. Alloys Compd., 2010, 493, 175.

170 L. A. Hutton, M. Vidotti, A. N. Patel, M. E. Newton, P. R. Unwin and J. V. MacPherson, J. Phys. Chem. C, 2011, 115, 1649.
171 D. M. MacArthur, J. Electrochem. Soc., 1970, 117, 422.

172 D. M. MacArthur, J. Electrochem. Soc., 1970, 117, 729.

173 H. Bode, K. Dehmelt and J. Witte, Electrochim. Acta, 1966, 11, 1079.

174 R. G. Egdell, J. B. Goodenough, A. Hamnett and C. J. Naish, J. Chem. Soc., Faraday Trans. 1, 1983, 79, 893.

175 L. D. Burke, M. E. G. Lyons and D. P. Whelan, J. Electroanal. Chem., 1982, 139, 131.

176 L. D. Burke and D. P. Whelan, J. Electroanal. Chem., 1984, 162, 121.

177 L. D. Burke and T. A. M. Twomey, J. Electroanal. Chem., 1984, 162, 101.

178 L. D. Burke and J. F. Healy, J. Electroanal. Chem., 1981, 124, 327.

179 F. A. Cotton and G. Wilkinson, Advanced Inorganic Chemistry, Wiley Interscience, New York, 3rd edn, 1972, pp. 644-646.

180 J. Burgess, Metal Ions in Solution, Wiley, New York, 1978, p. 266.

181 E. Guerrini, H. Chen and S. Trasatti, J. Solid State Electrochem., 2007, 11, 939.

182 O. J. Murphy, PhD thesis, University College Cork, 1981.

183 Handbook of Chemistry and Physics, ed. R. C. Weast, CRC Press, Cleveland, Ohio, 51st edn, 1970.

184 J. O'M. Bockris and T. Otagawa, J. Electrochem. Soc., 1984, 131, 290.

185 J. O’M. Bockris, T. Otagawa and V. Yound, J. Electroanal. Chem., 1983, 150, 633.

186 L. Ojefors, J. Electrochem. Soc., 1976, 123, 1691.

187 D. D. MacDonald and D. Owen, J. Electrochem. Soc., 1973, 120, 317.

188 H. Neugebauer, G. Nauer, N. Brinda-Konopik and G. Gidaly, J. Electroanal. Chem., 1981, 122, 381.

189 F. Beck, R. Kaus and M. Oberst, Electrochim. Acta, 1985, 30, 173.

190 R. S. S. Guzman, J. R. Vilche and A. J. Arvia, Electrochim. Acta, 1979, 24, 395.

191 S. Juanto, R. S. S. Guzman, J. O. Zerbino, J. R. Vilche and A. J. Arvia, Electrochim. Acta, 1991, 36, 1143.

192 G. Larramona and C. Gutierrez, J. Electrochem. Soc., 1989, 136, 2171.

193 R. Šimpraga and B. E. Conway, J. Electroanal. Chem., 1991, 313, 161.

194 S. T. Amaral, E. M. A. Martini and I. L. Muller, Corros. Sci., 2001, 43, 853.

195 S. Joiret, M. Keddam, X. R. Novoa, M. C. Perez, C. Rangel and H. Takenouti, Cem. Concr. Compos., 2002, 24, 7.

196 P. W. Schindler, in Adsorption of Inorganics at Solid-Liquid Interfaces, ed. M. A. Anderson and A. J. Rubin, Ann Arbor Science Publishers, Michigan, USA, ch. 1, 1981.

197 J. O’M. Bockris, M. Genshaw and V. Brusic, Symp. Faraday Soc., 1970, 4, 177.

198 J. O’M. Bockris, V. Brusic and H. Wroblowa, Electrochim. Acta, 1971, 16, 1859.

199 H. Wroblowa, V. Brusic and J. O'M. Bockris, J. Phys. Chem., 1971, 75, 2823. 
200 R. D. Armstrong and I. Baurhoo, J. Electroanal. Chem., 1972, 34, 41.

201 R. D. Armstrong and I. Baurhoo, J. Electroanal. Chem., 1972, 40, 325.

202 T. E. Pou, O. J. Murphy, V. Young, J. O'M. Bockris and L. L. Tongson, J. Electroanal. Chem., 1984, 131, 1243.

203 V. Jovancicevic, R. C. Kainthla, Z. Tang, B. Yang and J. O'M. Bockris, Langmuir, 1987, 3, 388.

204 W. E. O’Grady, J. Electrochem. Soc., 1980, 127, 555.

205 M. E. G. Lyons, Electroactive Polymer Electrochemistry, Plenum Press, New York, 1994, ch. 2, p. 102.

206 N. Oyama and T. Ohsaka, Prog. Polym. Sci., 1995, 20, 761.

207 R. Kotz, C. Barbero and O. Haas, J. Electroanal. Chem., 1990, 296, 37.

208 L. D. Burke and O. J. Murphy, J. Electroanal. Chem., 1980, 109, 373.

209 J. McBreen, in Handbook of Battery Materials, ed. J. O. Besenhard, Wiley-VCH, Weinheim, 2007, ch. 6, p. 135.

210 A. Seghiouer, J. Chevalet, A. Barhoun and F. Lantelme, J. Electroanal. Chem., 1998, 442, 113.

211 L. D. Burke and T. A. M. Twomey, J. Electroanal. Chem., 1984, 167, 285.

212 W. Visscher and E. Barendrecht, J. Appl. Electrochem., 1980, 10, 269.

213 L. M. M. de Souza, F. P. Kong, F. R. McLarnon and R. H. Muller, Electrochim. Acta, 1997, 42, 1253.

214 R. Simpraga and B. E. Conway, J. Electroanal. Chem., 1990, 280, 341.

215 A. C. Makrides, J. Electrochem. Soc., 1966, 113, 1158.

216 M. Okuyama and S. Haruyama, Corros. Sci., 1974, 14, 1.

217 L. D. Burke, M. E. Lyons and O. J. Murphy, J. Electroanal. Chem., 1982, 132, 247.

218 M. E. G. Lyons, R. L. Doyle, I. Godwin, M. O'Brien and L. Russell, J. Electrochem. Soc., 2012, 159, H932.

219 M. Wehrens-Dijksma and P. H. L. Notten, Electrochim. Acta, 2006, 51, 3609.

220 P. Bernard, C. Gabrielli, M. Keddam, H. Takenouti, J. Leonardi and P. Blanchard, Electrochim. Acta, 1991, 36, 743.

221 G. T. Cheek and W. E. O'Grady, J. Electroanal. Chem., 1997, 421, 173.

222 G. Feuillade and R. Jacoud, Electrochim. Acta, 1969, 14, 1297.

223 P. Benson, G. W. D. Briggs and W. F. K. Wynne-Jones, Electrochim. Acta, 1964, 9, 275.

224 W. K. Behl and J. E. Toni, J. Electroanal. Chem., 1971, 31, 63.

225 R. P. Simpraga, J. Electroanal. Chem., 1993, 355, 79.

226 T. C. Liu, W. G. Pell and B. E. Conway, Electrochim. Acta, 1999, 44, 2829.

227 H. Gomez Meier, J. R. Vilche and A. J. Arvia, J. Electroanal. Chem., 1982, 138, 367.

228 D. Erts, E. Ahlberg, J. Asbjornsson, H. Olin and J. Prikulis, J. Appl. Phys., 1998, A66, S477.

229 T. Ohtsuka and N. Sato, J. Electroanal. Chem., 1983, 147, 167.
230 K. E. Heusler, in Passivity of Metals, ed. R. P. Frankenthal and J. Kruger, Electrochem. Soc., Princeton NJ, 1978, p. 771.

231 A. Foelske and H. H. Strehblow, Surf. Interface Anal., 2002, 34, 125.

232 L. D. Burke and M. M. Murphy, J. Electrochem. Soc., 1991, 138, 88.

233 M. Gattrell and B. MacDougall, in Handbook of Fuel CellsFundamentals, Technology and Applications, ed. W. Vielsch, H. A. Gasteiger and A. Lamm, Wiley, vol. 2, 2003.

234 M. Zhi, C. Xiang, J. Li, M. Li and N. Wu, Nanoscale, 2013, 5,72 .

235 J. Jiang, Y. Li, J. Liu, X. Huang, C. Yuan and X. W. (D.) Lou, Adv. Mater., 2012, 24, 5166.

236 J. Bisquert, G. Garcia Belmonte, F. Fabregat Santiago, N. S. Ferriols, M. Yamashita and E. C. Pereira, Electrochem. Commun., 2000, 2, 601.

237 A. J. Terezo, J. Bisquert, E. C. Pereira and G. Garcia Belmonte, J. Electroanal. Chem., 2001, 508, 59.

238 J. Bisquert, G. Garcia-Belmonte, F. Fabregat-Santiago, N. S. Ferriols, P. Bogdanoff and E. C. Pereira, J. Phys. Chem. $B, 2000,104,2287$.

239 S. Sunde, I. A. Lervik, M. Tsypkin and L. E. Owe, Electrochim. Acta, 2010, 55, 7751.

240 L. Sziraki and L. Bobics, Electrochim. Acta, 2002, 47, 2189.

241 E. Fachinotti, E. Guerrini, A. C. Tavares and S. Trasatti, J. Electroanal. Chem., 2007, 600, 103.

242 R. W. Murray, in Molecular Design of Electrode Surfaces, ed. R. W. Murray, Techniques of Chemistry Series, Wiley Interscience, New York, 1992, vol. 22, ch. 1, p. 1.

243 J. C. Jernigan, C. E. D. Chidsey and R. W. Murray, J. Am. Chem. Soc., 1985, 107, 2824.

244 C. E. D. Chidsey and R. W. Murray, J. Phys. Chem., 1986, 90, 1479.

245 E. F. Dalton, N. A. Surridge, J. C. Jernigan, K. O. Wilbourn, J. S. Facci and R. W. Murray, Chem. Phys., 1990, 141, 143.

246 J. C. Jernigan, N. A. Surridge, M. E. Zvanut, M. Silver and R. W. Murray, J. Phys. Chem., 1989, 93, 4620.

247 E. Laviron, J. Electroanal. Chem., 1974, 52, 395.

248 E. Laviron, J. Electroanal. Chem., 1980, 112, 1.

249 E. Laviron, L. Roullier and C. Degrand, J. Electroanal. Chem., 1980, 112, 11.

250 E. Laviron, J. Electroanal. Chem., 1981, 122, 37.

251 E. Laviron and L. Roullier, J. Electroanal. Chem., 1980, 115, 65.

252 E. Laviron, J. Electroanal. Chem., 1979, 101, 19.

253 K. Aoki, K. Tokuda and H. Matsuda, J. Electroanal. Chem., 1983, 146, 417.

254 K. Aoki, K. Tokuda and H. Matsuda, J. Electroanal. Chem., 1984, 160, 33.

255 CRC Handbook of Chemistry and Physics, ed. D. R. Linde, CRC Press, 2007, 88th edn, pp. 4-68.

256 Encyclopedia of Minerals, ed. W. L. Roberts, T. J. Campbell and G. R. Rapp Jr, Van Nostrand Reinhold, New York, 2nd edn, 1990, p. 273.

257 I. Rubenstein, E. Sabatani and J. Rishpon, J. Electrochem. Soc., 1987, 134, 3078. 
258 J. C. Lacroix, K. K. Kanazawa and A. Diaz, J. Electrochem. Soc., 1989, 136, 1308.

259 M. E. G. Lyons, H. G. Fay, T. McCabe, J. Corish, J. G. Vos and A. J. Kelly, J. Chem. Soc., Faraday Trans., 1990, 86, 2905.

260 R. M. Penner and C. R. Martin, J. Phys. Chem., 1989, 93, 984.

261 P. Burgmayer and R. W. Murray, J. Am. Chem. Soc., 1982, 104, 6139.

262 L. D. Burke, M. E. G. Lyons, E. J. M. O'Sullivan and D. P. Whelan, J. Electroanal. Chem., 1981, 122, 403.

263 N. T. Beukes and J. Badenhorst, J. South. Afr. Inst. Min. Metall., 2009, 109, 343.

264 Z. S. Msindo, V. Sibanda and J. H. Potgieter, J. Appl. Electrochem., 2010, 40, 691.

265 M. T. M. Koper, J. Electroanal. Chem., 2005, 574, 375.

266 M. T. M. Koper, Faraday Discuss., 2008, 140, 11.

267 C. A. Lucas, M. Cormack, M. E. Gallagher, A. Brownrigg, P. Thompson, B. Fowler, Y. Grunder, J. Roy, V. Stamenkovic and N. M. Markovic, Faraday Discuss., 2009, 140, 41.

268 H. Noguchi, T. Okada and K. Uosaki, Faraday Discuss., 2009, 140, 125.

269 D. J. Schiffrin, Faraday Discuss., 2009, 140, 439.

270 J. Rossmeisl, A. Logadottir and J. K. Norskov, Chem. Phys., 2005, 315, 178.

271 J. Rossmeisl, Z. W. Qu, H. Zhu, G. J. Kroes and J. K. Norskov, J. Electroanal. Chem., 2007, 607, 83.

272 J. K. Norskov, J. Rossmeisl, A. Logadottir, L. Lindqvist, J. R. Kitchin, T. Bligaard and H. Jonsson, J. Phys. Chem. B, 2004, 108, 17886.

273 H. A. Hansen, I. C. Man, F. Studt, F. Abild-Pedersen, T. Bligaard and J. Rossmeisl, Phys. Chem. Chem. Phys., 2010, 12, 283.

274 A. J. Bard and L. R. Faulkner, Electrochemical Methods: Fundamentals and Applications, Wiley, New York, 2nd edn, 2000.

275 J. P. Hoare, The Electrochemistry of Oxygen, Interscience, New York, 1968, pp. 82-91.

276 A. Damjanovic, M. A. Genshaw and J. O'M. Bockris, J. Electrochem. Soc., 1967, 114, 466.

277 A. Damjanovic, M. A. Genshaw and J. O'M. Bockris, J. Electrochem. Soc., 1967, 114, 1107.

278 R. Parsons, Trans. Faraday Soc., 1951, 47, 1332.

279 J. O'M. Bockris and S. U. M. Khan, Surface Electrochemistry, Plenum Press, New York, 1993, Section 3.5, pp. 218-223.

280 A. Damjanovic, A. Dey and J. O'M. Bockris, Electrochim. Acta, 1966, 11, 791.

281 M. E. G. Lyons and M. P. Brandon, J. Electroanal. Chem., 2010, 641, 119.

282 A. Hrussanova, E. Guerrini and S. Trasatti, J. Electroanal. Chem., 2004, 564, 151.

283 W. Oelßner, F. Berthold and U. Guth, Mater. Corros., 2006, $57,455$.

284 D. Britz, J. Electroanal. Chem., 1978, 88, 309.

285 R. A. Robinson and R. H. Stokes, Electrolyte Solutions, Butterworth \& Co. Ltd., London, 1965, p. 492.
286 R. Parsons, in Advances in Electrochemistry and Electrochemical Engineering, ed. P. Delahay, Interscience, New York, 1961, vol. 1, p. 1.

287 B. E. Conway and M. Salomon, Electrochim. Acta, 1964, 9, 1599.

288 W. J. Albery, Electrode Kinetics, Clarendon Press, Oxford, 1975 , p. 41.

289 J. O’M. Bockris, A. K. N. Reddy and M. Gamboa-Aldeco, Modern Electrochemistry 2A, Kulwer Academic Publishers, New York, 2nd edn, 2002, p. 1412.

290 B. E. Conway and P. L. Bourgault, Trans. Faraday Soc., 1962, 58, 593.

291 B. E. Conway and P. L. Bourgault, Can. J. Chem., 1959, 37, 292.

292 P. L. Bourgault and B. E. Conway, Can. J. Chem., 1960, 38, 1557.

293 M. E. G. Lyons and M. P. Brandon, J. Electroanal. Chem., 2009, 631, 62.

294 D. A. Harrington and B. E. Conway, Electrochim. Acta, 1987, 32, 1703.

295 B. Klahr, S. Gimenez, F. Fabregat-Santiago, J. Bisquert and T. W. Hamann, J. Am. Chem. Soc., 2012, 134, 16693.

296 H. Willems, A. G. C. Kobussen, J. H. W. De Wit and G. H. J. Broers, J. Electroanal. Chem., 1984, 170, 227.

297 J. Z. Giner, Z. Elektrochem., 1959, 63, 386.

298 K. Kinoshita, Electrochemical Oxygen Technology, Wiley, New York, ch. 2, 1992.

299 A. I. Krasil'shchikov, Zh. Fiz. Khim., 1963, 37, 531.

300 W. O'Grady, C. Iwakura, J. Huang and E. Yeager, in Proceedings of the Symposium on Electrocatalysis, ed. M. W. Breiter, The Electrochemical Society Inc., Pennington, NJ, 1974, p. 286.

301 A. G. C. Kobussen and G. H. J. Broers, J. Electroanal. Chem., 1981, 126, 221.

302 S. Trasatti, Handbook of Fuel Cells, Fundamentals Technology and Applications, in Electrocatalysis, ed. W. Vielstich, A. Lamm and H. A. Gasteiger, John Wiley and Sons, Chichester, 2003, vol. 2, ch. 10.

303 J. Suntivich, K. J. May, H. Gasteiger, J. B. Goodenough and Y. Shao-Horn, Science, 2011, 334, 1383.

304 R. Subbaraman, D. Tripkovic, K.-C. Chang, D. Strmcnik, A. P. Paulikas, P. Hirunsit, M. Chan, J. Greeley, V. Stamenkovic and N. M. Markovic, Nat. Mater., 2012, 11, 550 .

305 S. W. Gestern, G. J. Samuels and T. J. Meyer, J. Am. Chem. Soc., 1982, 104, 4029.

306 J. A. Gilbert, D. S. Eggleston, J. W. R. Murphy, D. A. Geselowitz, S. W. Gestern, D. J. Hodgson and T. J. Meyer, J. Am. Chem. Soc., 1985, 107, 3855.

307 J. J. Concepcion, J. W. Jurss, J. L. Templeton and T. J. Meyer, Proc. Natl. Acad. Sci. U. S. A., 2008, 105, 17632.

308 J. J. Concepcion, J. W. Jurss, M. K. Brennaman, P. G. Hoertz, A. O. T. Patrocinio, N. Y. M. Iha, J. L. Templeton and T. J. Meyer, Acc. Chem. Res., 2009, 42, 1954.

309 R. Zong and R. P. Thummel, J. Am. Chem. Soc., 2005, 127, 12802. 
310 R. Z. H.-W. Tseng, J. T. Muckerman and R. Thummel, Inorg. Chem., 2008, 47, 11763.

311 G. Zhang, R. Zong, H.-W. Tseng and R. P. Thummel, Inorg. Chem., 2008, 47, 990.

312 S. Romain, F. Bozoglian, X. Sala and A. Llobet, J. Am. Chem. Soc., 2009, 131, 2768.

313 F. Bozoglian, S. Romain, M. Z. Ertem, T. K. Todorova, C. Sens, J. Mola, M. Rodrguez, I. Romero, J. Benet Buchholz, X. Fontrodona, C. J. Cramer, L. Gagliardi and A. Llobet, J. Am. Chem. Soc., 2009, 131, 15176.

314 X. Sala, I. Romero, M. Rodriguez, L. Escriche and A. Llobet, Angew. Chem., 2009, 121, 2882.

315 X. Sala, M. Z. Ertem, L. Vigara, T. K. Todorova, W. Chen, R. C. Rocha, F. Aquilante, C. J. Cramer, L. Gagliardi and A. Llobet, Angew. Chem., Int. Ed., 2010, 49, 7745.

316 L. Duan, F. Bozoglian, S. Mandal, B. Stewart, T. Privalov, A. Llobet and L. Sun, Nature, 2012, 4, 418.

317 L. Duan, A. Fischer, Y. Xu and L. Sun, J. Am. Chem. Soc., 2009, 131, 10397.

318 L. Duan, Y. Xu, P. Zhang, M. Wang and L. Sun, Inorg. Chem., 2010, 49, 209.

319 J. Nyhlen, L. Duan, B. Akermark, L. Sun and T. Privalov, Angew. Chem., 2010, 122, 1817.

320 C. W. Cady, R. H. Crabtree and G. W. Brudvig, Coord. Chem. Rev., 2008, 252, 444.

321 J. F. Hull, D. Balcells, J. D. Blakemore, C. D. Incarvito, O. Eisenstein, G. W. Brudvig and R. H. Crabtree, J. Am. Chem. Soc., 2009, 131, 8730.

322 P. E. M. Siegbann and R. H. Crabtree, J. Am. Chem. Soc., 1999, 121, 117.

323 M. Busch, E. Ahlberg and I. Panas, Phys. Chem. Chem. Phys., 2011, 13, 15069.

324 L.-P. Wang, Q. Wu and T. Van Voorhis, Inorg. Chem., 2010, 49, 4543.

325 J. P. Hoare, Adv. Electrochem. Electrochem. Eng., 1967, 6, 201.

326 L. D. Burke, M. E. G. Lyons and M. McCarthy, Adv. Hydrogen Energy, 1982, 3, 267.

327 N. Sato, Electrochemistry at Metal and Semiconductor Electrodes, Elsevier, 1998, pp. 181-184.

328 E. J. M. O’Sullivan and L. D. Burke, J. Electrochem. Soc., 1990, 137, 466.
329 P. Vassilev, M. T. M. Koper and R. A. van Santen, Chem. Phys. Lett., 2002, 359, 337.

330 M. Tuckerman, K. Laasonen, M. Sprik and M. Parrinello, J. Chem. Phys., 1995, 103, 150.

331 D. Marx, M. E. Tuckerman, J. Hutter and M. Parrinello, Nature, 1999, 397, 601.

332 J. J. McDonald and B. E. Conway, Proc. R. Soc. London, Ser. A, 1962, 269, 419.

333 R. E. Meyer, J. Electrochem. Soc., 1960, 107, 847.

334 M. M. Lohrengel and J. W. Schultze, Electrochim. Acta, 1976, 21, 957.

335 X. Shen, Y. A. Small, J. Wang, P. B. Allen, M. V. FernandezSerra, M. S. Hybertsen and J. T. Muckerman, J. Phys. Chem. $C, 2010,114,13695$.

336 G. A. Lawrence, Introduction to Coordination Chemistry, J. Wiley \& Sons, West Sussex, 2010, p. 199.

337 A. R. McDonald and L. Que, Nature, 2011, 3, 761.

338 B. S. Yeo, S. L. Klaus, P. N. Ross, R. A. Mathies and A. T. Bell, ChemPhysChem, 2010, 11, 1854.

339 D. Cibrev, M. Jankulovska, T. Lana-Villarreal and R. Gomez, Int. J. Hydrogen Energy, 2013, 38, 2746.

340 G. C. Dismukes, R. Brimblecombe, G. A. N. Felton, R. S. Pryadun, J. E. Sheats, L. Spiccia and G. F. Swiegers, Acc. Chem. Res., 2009, 42, 1935.

341 R. Brimblecombe, C. Dismukes, G. F. Swiegers and L. Spiccia, Dalton Trans., 2009, 43, 9374.

342 J. Feng, Angew. Chem., 2010, 48, 1841.

343 T. Schmidt and H. Wendt, Electrochim. Acta, 1994, 39, 1763.

344 A. Harriman, I. J. Pickering, J. M. Thomas and P. A. Christensen, J. Chem. Soc., Faraday Trans. 1, 1988, 84, 2795.

345 M. Hara, J. T. Lean and T. E. Mallouk, Chem. Commun., 2001, 13, 4668.

346 N. D. Morris, M. Suzuki and T. E. Mallouk, J. Phys. Chem. A, 2004, 108, 9115.

347 P. G. Hoertz, Y. I. Kim, W. J. Youngblood and T. E. Mallouk, J. Phys. Chem. B, 2007, 111, 6845.

348 M. Yagi, E. Tomita, S. Sakita, T. Kuwabara and K. Nagai, J. Phys. Chem. B, 2005, 109, 21489.

349 M. Morita, C. Iwakura and H. Tamura, Electrochim. Acta, 1977, 22, 325.

350 X. Zhang, J. Leddy and A. J. Bard, J. Am. Chem. Soc., 1985, 107, 3715. 